\title{
WestVirginiaUniversity
}

THE RESEARCH REPOSITORY @ WVU

Graduate Theses, Dissertations, and Problem Reports

2011

\section{Hardware Prototype for a Multi Agent Grid Management System}

Jerry Lee Thompson

West Virginia University

Follow this and additional works at: https://researchrepository.wvu.edu/etd

\section{Recommended Citation}

Thompson, Jerry Lee, "Hardware Prototype for a Multi Agent Grid Management System" (2011). Graduate Theses, Dissertations, and Problem Reports. 579.

https://researchrepository.wvu.edu/etd/579

This Thesis is protected by copyright and/or related rights. It has been brought to you by the The Research Repository @ WVU with permission from the rights-holder(s). You are free to use this Thesis in any way that is permitted by the copyright and related rights legislation that applies to your use. For other uses you must obtain permission from the rights-holder(s) directly, unless additional rights are indicated by a Creative Commons license in the record and/ or on the work itself. This Thesis has been accepted for inclusion in WVU Graduate Theses, Dissertations, and Problem Reports collection by an authorized administrator of The Research Repository @ WVU. For more information, please contact researchrepository@mail.wvu.edu. 


\title{
Hardware Prototype for a Multi Agent Grid Management System
}

\author{
Jerry Lee Thompson \\ Thesis submitted to the \\ College of Engineering and Mineral Resources at West \\ Virginia University \\ in partial fulfillment of the requirements for the \\ degree of \\ Master of Science \\ in \\ Electrical Engineering
}

Prof. Ali Feliachi, Ph.D., Chair

Prof. Powsiri Klinkhachorn, Ph.D.

Prof. Mohammed A. Choudhry, Ph.D.

Lane Department of Computer Science and Electrical Engineering

Morgantown, West Virginia

2011

Keywords: Smart Grid, Power System, Reconfigurable Power System, Agent Communication, Multi-Agent System, Fault Detection, Reconfiguration 


\title{
Abstract \\ Hardware Prototype for a Multi Agent Grid Management System
}

\author{
Jerry Lee Thompson
}

There is great effort in the power industry to incorporate Smart Grid functionalities to existing power systems. Distributed generation and the hardware necessary to interface the existing grid, as well as control algorithms to efficiently couple and operate these systems are being researched and implemented extensively. However, the added complexity of such components results in greater opportunities for failure in a system which is already challenging to protect.

To aid in the comprehension and predictive ability of protection practices, software simulations are run extensively. This not only provides insights upon scenarios which may not occur regularly, but also eliminates the risk of damaging expensive equipment. Yet, software simulation is limited in that it does not incorporate the entire set of physical rules which govern the system. When considering the complicated dynamics of a power system, even reasonably detailed models require extensive time for evaluation. Modeling a power system in full detail is simply impractical for all but the most powerful and expensive of computer systems. In remedy of this fact, the Power System Simulator at WVU is a scaled replica of a distribution power system. It is able to accurately portray the responses of a power system in full detail at an operator's whim.

Built in the late 1970's, the Power System Simulator in its original configuration provides an antiquated version of a protection system. Electromechanical relays and switches yield reconfiguration capabilities with relatively little input from an operator. With the objectives of a Smart Grid in mind, the Power System Simulator is upgraded to provide a fully function test bed capable of utilizing the entire infrastructure available to utilities. This includes digital relays with advanced metering capability, hosting for complex algorithms, and user-defined reconfiguration capabilities.

This thesis will explore work done to the simulator transforming it into a device capable of testing Smart Grid applications in an environment which encapsulates all the laws of physics, giving insight to operation and unveiling previously unexplored challenges. Similarly, this also provides a proving ground for many of the complicated algorithms which are being investigated with the objective to optimize the performance of a distribution system. 


\section{Dedication}

This thesis is dedicated to

my parents, Gerald and Debra Thompson

my wife, Mary Thompson 


\section{Acknowledgement}

I would like to thank the entire faculty of the Lane Computer Science and Electrical Engineering department at WVU. Not only have they imparted their knowledge and intuition, but my time here has been thoroughly enjoyable through the enthusiasm and compassion of many of my professors. I feel very fortunate to have studied under so many great minds with rich personal caliber.

More specifically, I would like to thank the members of my committee. As an undergraduate student, Dr. Mohammed Choudhry was a driving force in encouraging me to complete my Master's degree. A superb professor, his courses always focused on practical and interesting material. He has been an invaluable aid with the many aspects of my education. My advisor, Dr. Ali Feliachi, has been similarly impacting on my academic career. His support, motivation, and insight have allowed me to focus on the aspects of electrical engineering which I find the most interesting and rewarding. The coursework and projects offered by Dr. Feliachi have been crucial in developing my skills as a power engineer. Both deserve the greatest and sincerest of gratitude. Dr. Klinkhchorn has also provided a wealth of knowledge which I have used in the scope of this thesis and beyond. His continued encouragement and resourcefulness have proved helpful time and time again.

Furthermore, I would like to thank the APERC team for their support and enthusiasm over the past two years. Dr. John Saymanki, Dr. Ed Sneckenberger, Dr. Rabie Belkacemi, Adesola Ademoye, and Mansa Chenurri have yielded great resource, and have been a true pleasure to work with. 


\section{Table of Contents}

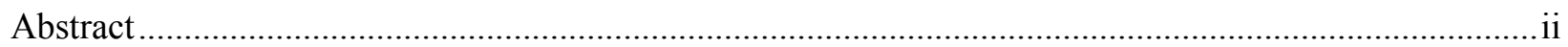

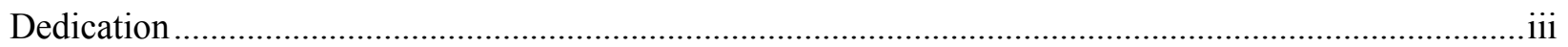

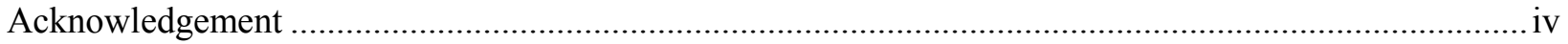

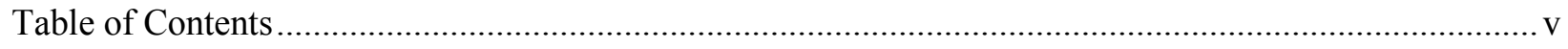

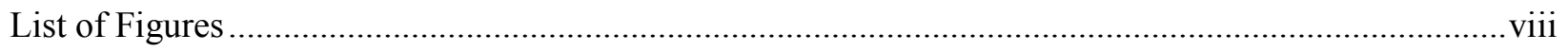

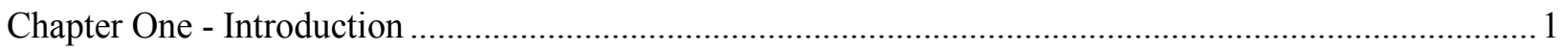

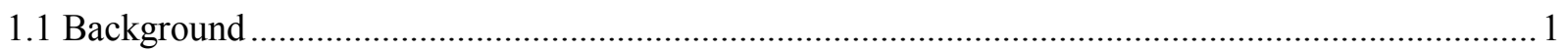

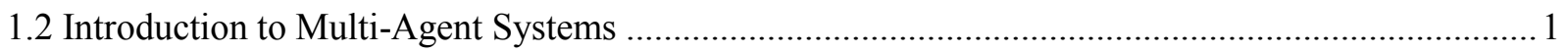

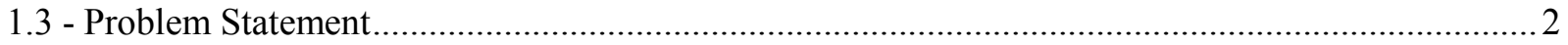

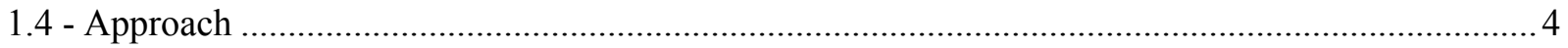

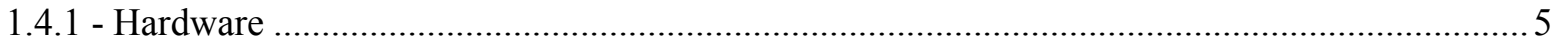

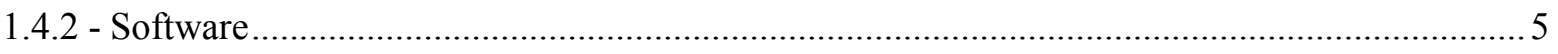

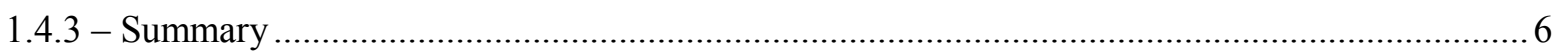

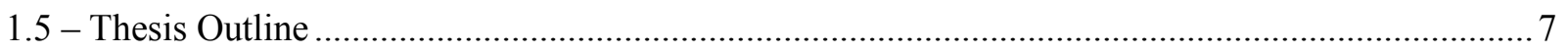

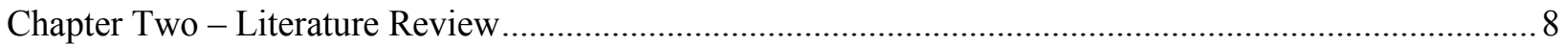

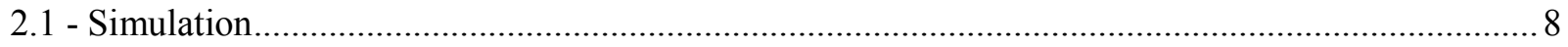

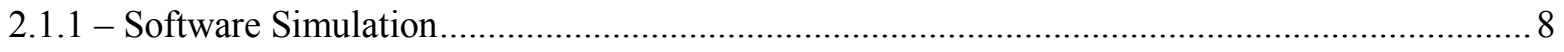

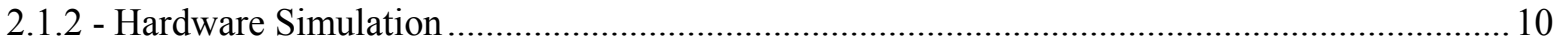

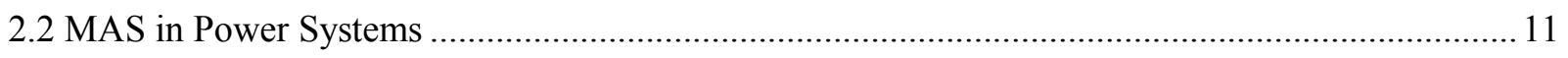

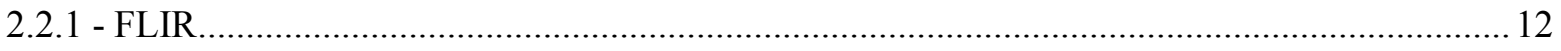

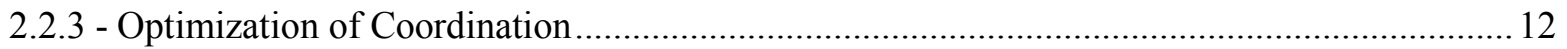

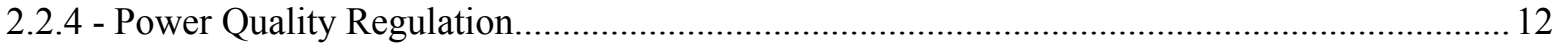

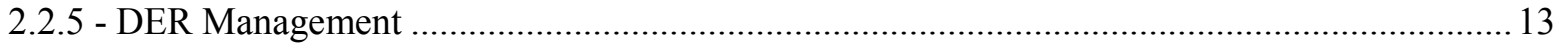

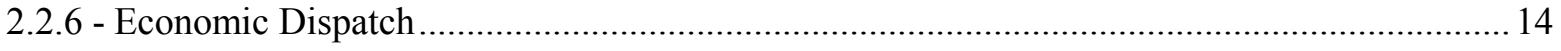




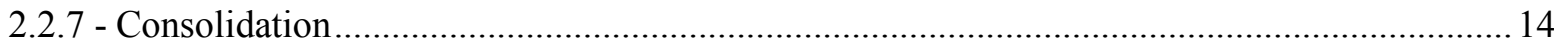

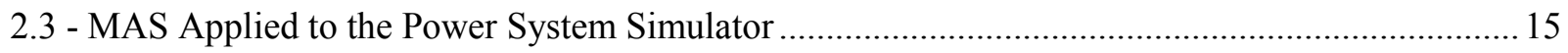

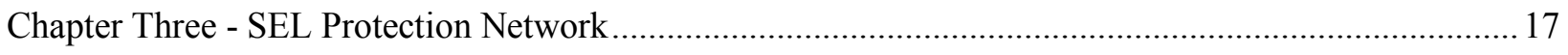

3.1 - Protection System Hardware for Distribution Networks ............................................................ 17

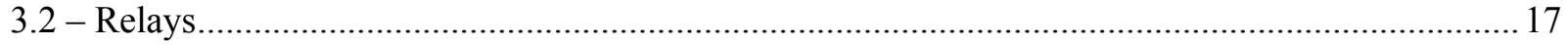

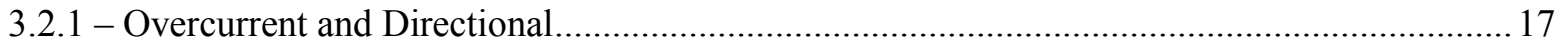

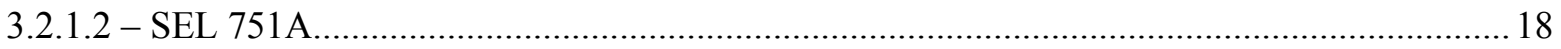

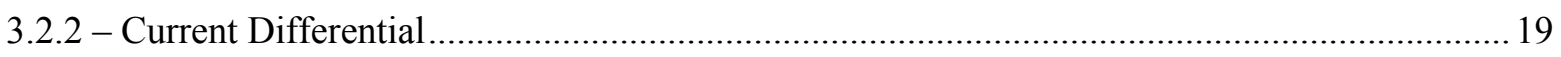

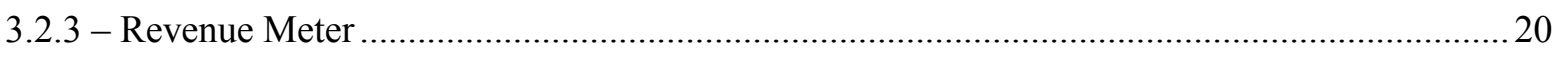

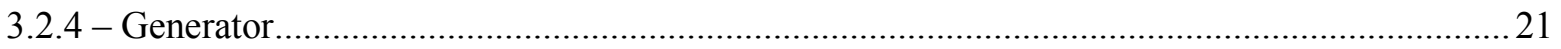

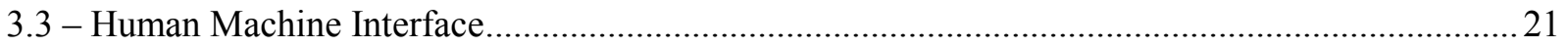

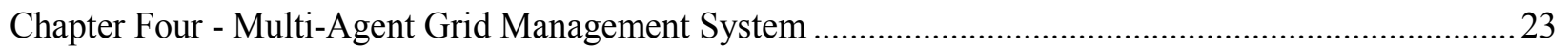

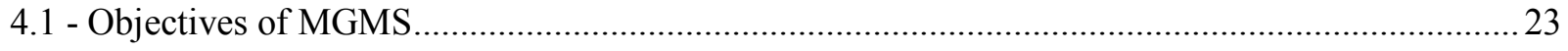

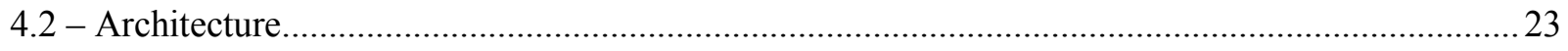

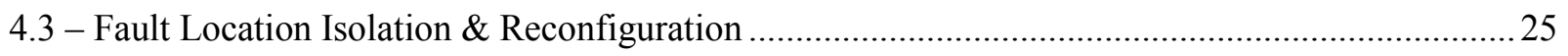

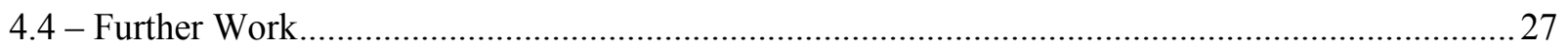

Chapter Five - Freescale Codewarrior Studio and Applications............................................................ 30

5.1 Freescale Codewarrior Integrated Development Environment .................................................... 30

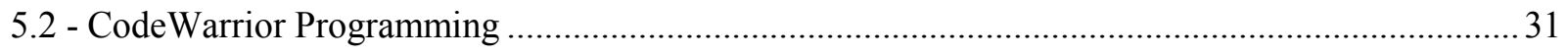

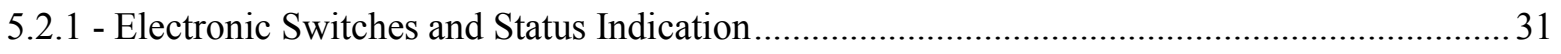

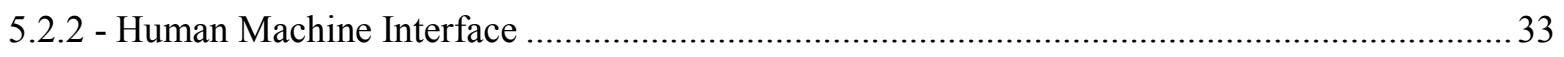

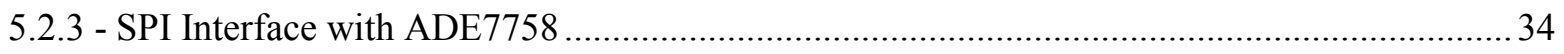

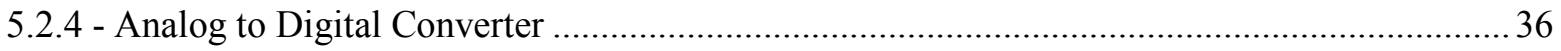

Chapter Six - Hardware Integration Results.............................................................................. 37

6.1 - Integration of SEL Devices to the Power System Simulator ..................................................... 37

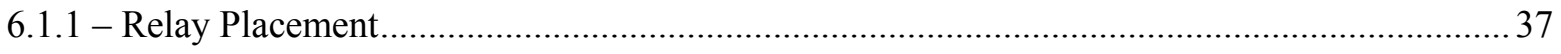

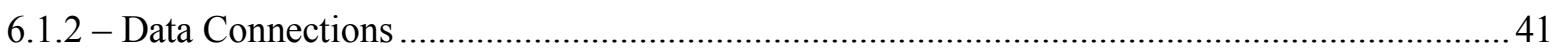




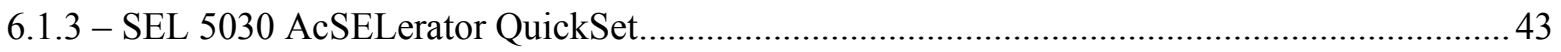

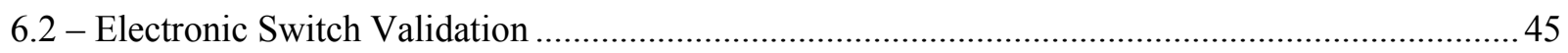

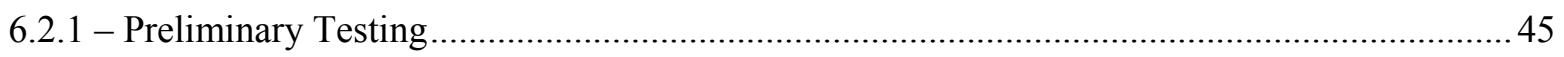

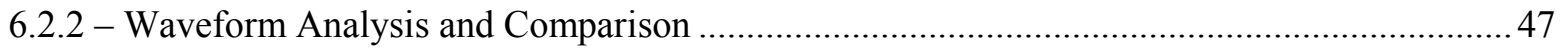

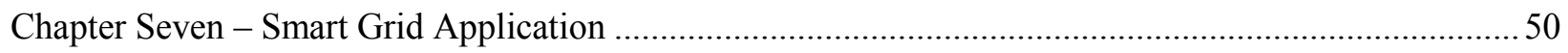

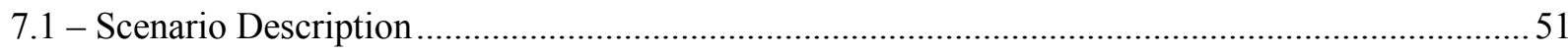

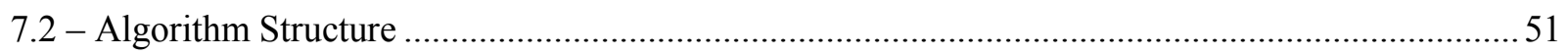

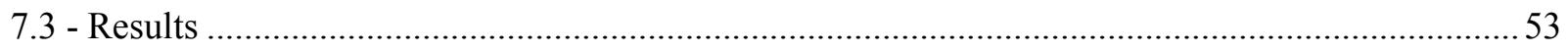

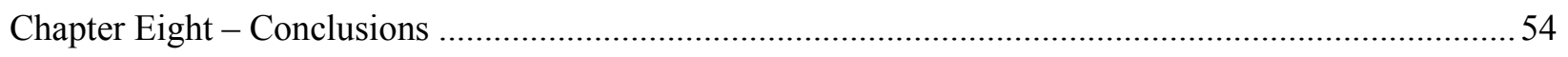

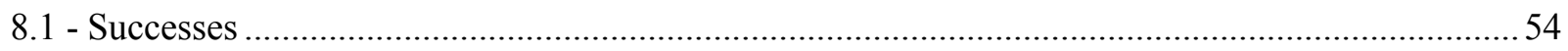

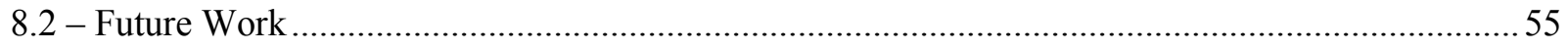

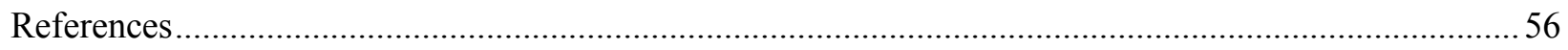

Appendix A: SEL Relay Demonstration Laboratory …................................................................5

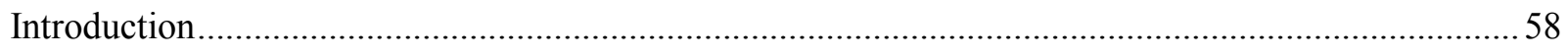

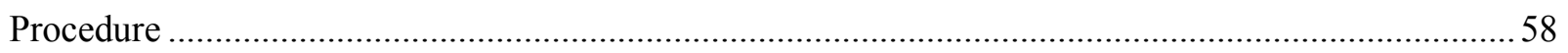




\section{List of Figures}

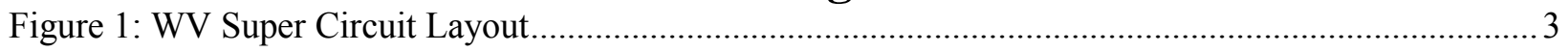

Figure 2: Power System Simulator in Original Configuration............................................................. 5

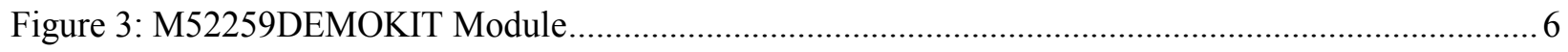

Figure 4: CYME GUI (CYME Power Engineering Software \& Solutions, 2011) ................................... 8

Figure 5: Real Time Digital Simulator Rack (RTDS Hardware, 2011) ................................................. 9

Figure 6: Hardware Simulation of Shipboard Power System .............................................................. 10

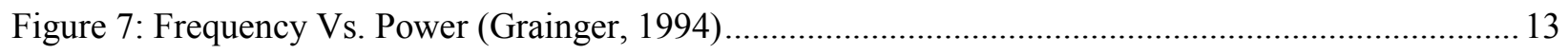

Figure 8: Commonly Used Distributed Energy Resources (Barney L. Capehart, 2010) ......................... 13

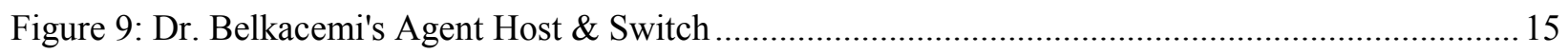

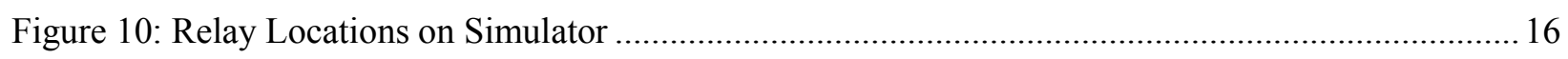

Figure 11: SEL 351 and 351S Overcurrent Protection Relays (Schweitzer Engineering Laboratories,

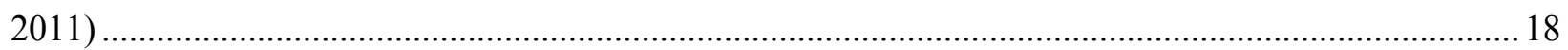

Figure 12: SEL-751A (Schweitzer Engineering Laboratories, 2011) .................................................... 18

Figure 13: SEL-387A (Schweitzer Engineering Laboratories, 2011) ................................................... 19

Figure 14: SEL-734P (Schweitzer Engineering Laboratories, 2011) ....................................................20

Figure 15: SEL 3351 (Schweitzer Engineering Laboratories, 2011) ....................................................22

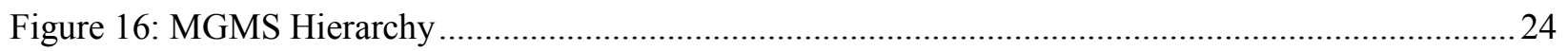

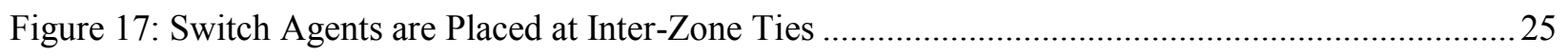

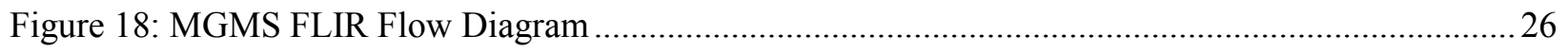

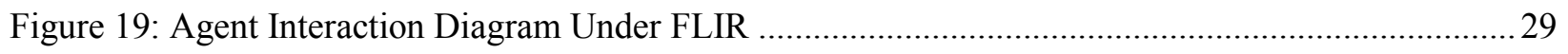

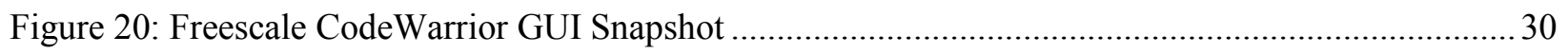

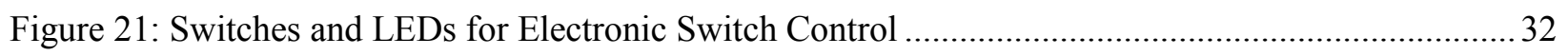

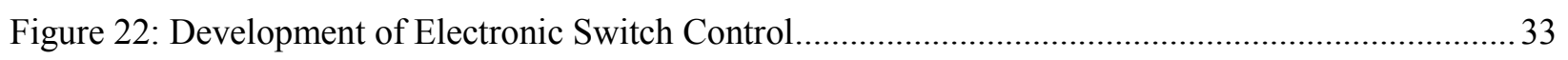

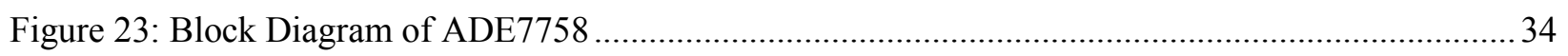

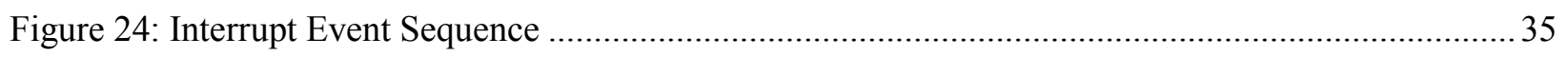

Figure 25: SEL Relays Integrated to the Power System Simulator (Spencer, 2010) ............................... 37

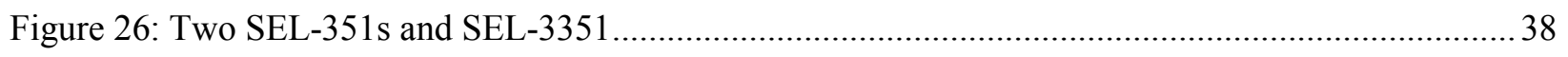

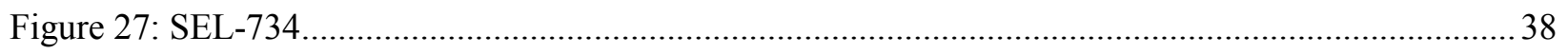

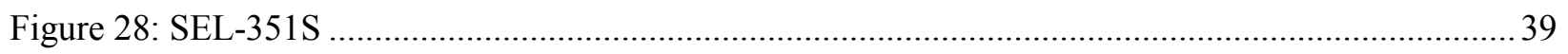

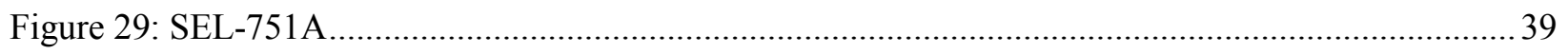

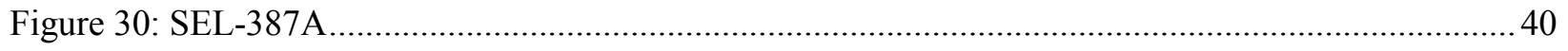

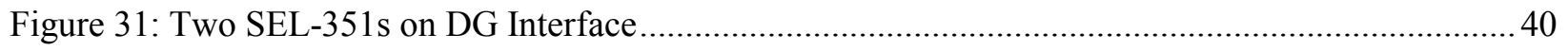

Figure 32: RS-232 Connections on Back Panel of SEL-3351 ........................................................... 41 


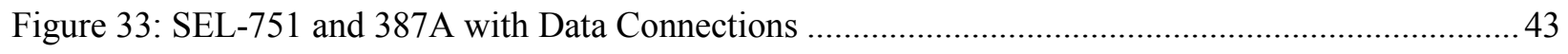

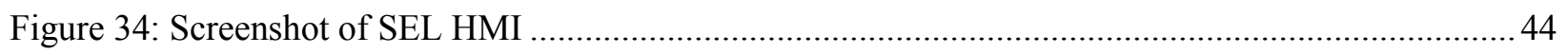

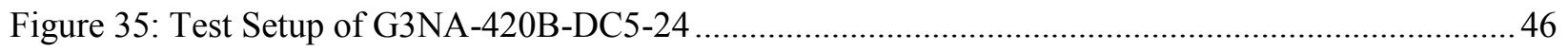

Figure 36: Solid State Relay Deployed to the Power System Simulator ................................................ 47

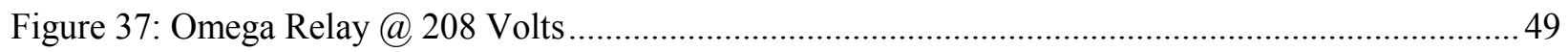

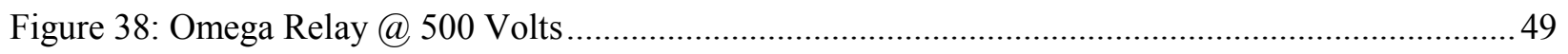

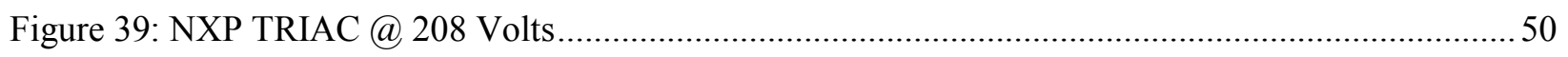

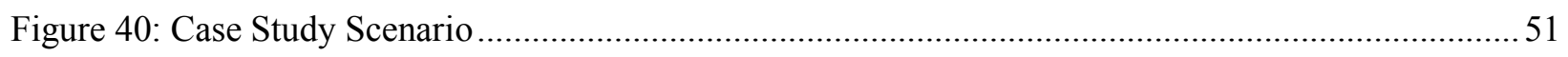

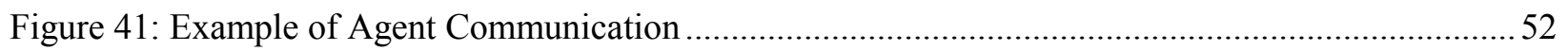




\section{Chapter One - Introduction}

\subsection{Background}

Due to the relatively inexpensive and versatile nature of computer simulation, its use is ubiquitous throughout all engineering disciplines. Theory of operation can be verified quickly and economically, without investing large amounts of capital into possibly unnecessary prototypes. With the high cost of power system infrastructure and critical nature of service, simulation becomes an incredibly powerful tool when minimizing costs incurred to a utility.

Even though countless scenarios can be modeled using software simulation, some cases require even further proof of concept before deployment is practical. When human life or excessive amounts of monetary value is jeopardized, extensive testing is mandatory. Such an instance is a distribution power system. Not only do lives possibly hang on the line (health systems without back-up), but a cataclysmic failure could put an entire region's economic activity to a halt. Small scale hardware platforms must be developed to explore unimagined contingencies in non-ideal settings, as well as discover unintended consequences to accepted control strategies.

\subsection{Introduction to Multi-Agent Systems}

As known in the field of Artificial Intelligence, an Intelligent Agent is an autonomous entity which acts upon an environment and directs its activity towards achieving goals. Agents may learn or use knowledge to achieve their goals (Intelligent Agent, 2009). A Multi-Agent System (MAS) is a network consisting of many Intelligent Agents which collaborate to achieve a similar or identical goal. In general, the objectives to which MASs are applied are extremely difficult or impossible for a single entity to achieve on its own.

There are several defining properties of a MAS (Multi-Agent System, 2011):

- Autonomy

- Limited Perspective

- Decentralization

Elaborating on the notes above, each agent in the system has at least a degree of autonomy. No agent is completely controlled by a (human) user. Generally speaking, agents do not have access to a global view of their environment; often their senses and actuators only pertain to a small portion of the 
system. Furthermore, no agent is a dedicated 'overseer.' That is, no agent is a central control node for other agents; a MAS is decentralized by nature.

For the purposes of this thesis, the basic descriptions of agents and MASs above will suffice, though further detail will be examined in subsequent chapters.

\section{3 - Problem Statement}

Mon Power, a power utility in Morgantown, WV has put forth significant effort to create a SmartGrid demonstration hosted on the local distribution network. With contributions from university research teams, the company intends to realize experimental strategies such as Distributed Generation, Demand Response, Micro-Grids, and Multi-Agent Systems.

The original West Virginia Super Circuit project consists of eight interconnected zones. Each zone is connected to at least one other zone. To power these zones, several feeders supply from two separate substations on different sides of the city (see Figure 1). One of the many objectives is to implement an automated Fault Location, Isolation, and Reconfiguration algorithm. Along with other supporting roles, this particular task falls into the capable hands of WVU's Advanced Power and Energy Research Center.

The WVSC collaborative research effort provides an ideal environment for academic institutes to coordinate with industry concerns in order to produce an effective and practical control system which can be exported for use in Smart-Grid implementations. 


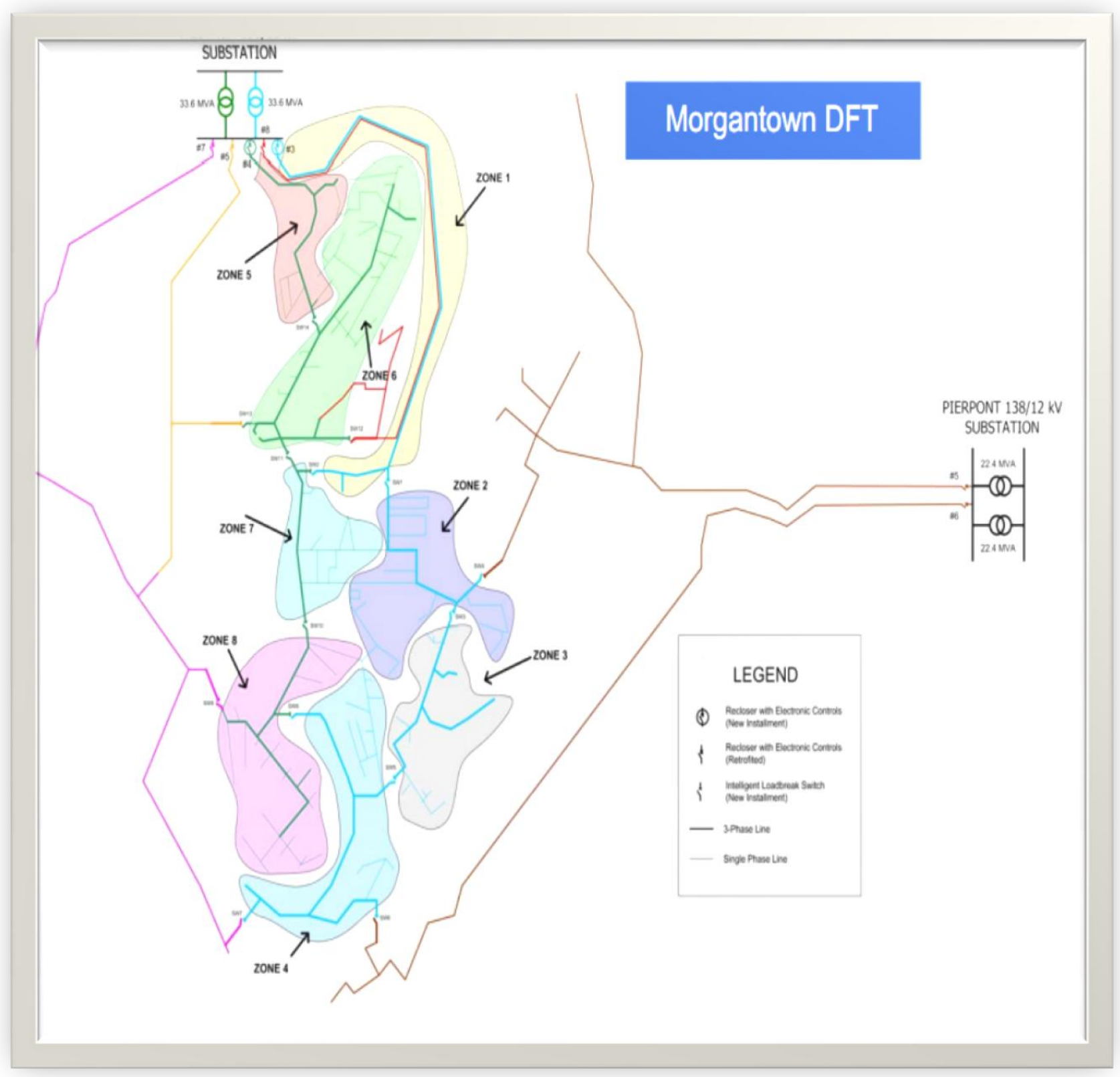

Figure 1: WV Super Circuit Layout 


\section{4 - Approach}

Each of the zones in the WVSC is connected to at least one other zone by switches, identified by paths crossing from one zone into another. Each zone can be electrically isolated from the rest of the system through these switches. Switch locations will serve as convenient points for data acquisition and algorithm hosting. By sampling data at these critical positions, any contingency in the system can be identified and controlled.

Though the concept is simple, the complexity of the system mandates a versatile solution. A fault in a distribution system can wreak havoc on an infrastructure if not quickly eliminated. Time constraint requires that the system be at least semi-autonomous. A geographically dispersed network bounds information exchange if the system is to adhere to the time frame. Following this notion, a decentralized control structure is necessary to maximize effectiveness with restrained time and data resources. All of these stipulations represent the strengths of a Multi-Agent System.

WVU's Advanced Power and Electricity Research Center's (APERC) deliverables in the project center around Multi-Agent Systems; more specifically, a MAS-based Fault Location, Isolation, and Reconfiguration algorithm. Software requirements, a scalable architecture, and other application design considerations are of critical concern. However, because of immediate proximity and resources available at WVU, APERC is also the spearhead for developing hardware platforms and the integration which will stand as the proof of concept for these MAS operations in industry settings.

In order to fully engage all aspects of a Smart Grid, there must be two distinct efforts: hardware and software. The hardware platform will provide the physical resources of a distribution system, as well as devices to host the software system. The software system will provide interface to human operators, manage the resources of the hardware system, and sample operating conditions of the system.

As with any experimental technologies, an amount of risk is involved until the experimental processes can become proven and refined. In order to provide the utility with a low probability of failure, APERC has focused on developing hardware and software solutions specific to the project, yet applicable to any situation. This thesis will explore aspects of the solution designed to mimic industrial operations and practices. 


\subsection{1 - Hardware}

The Hampden Engineering Corporation Power System Simulator is an integral strength of the hardware platform. The original system is a replica of a distribution system. Relays and breakers are placed throughout the system and are mounted for observation and easy access. Hand switches are also installed at many locations along the lines so that power can be routed as the operator finds desirable. The Power System Simulator provides the test bed which will validate the MASs that APERC designs in fulfillment of the project objectives.

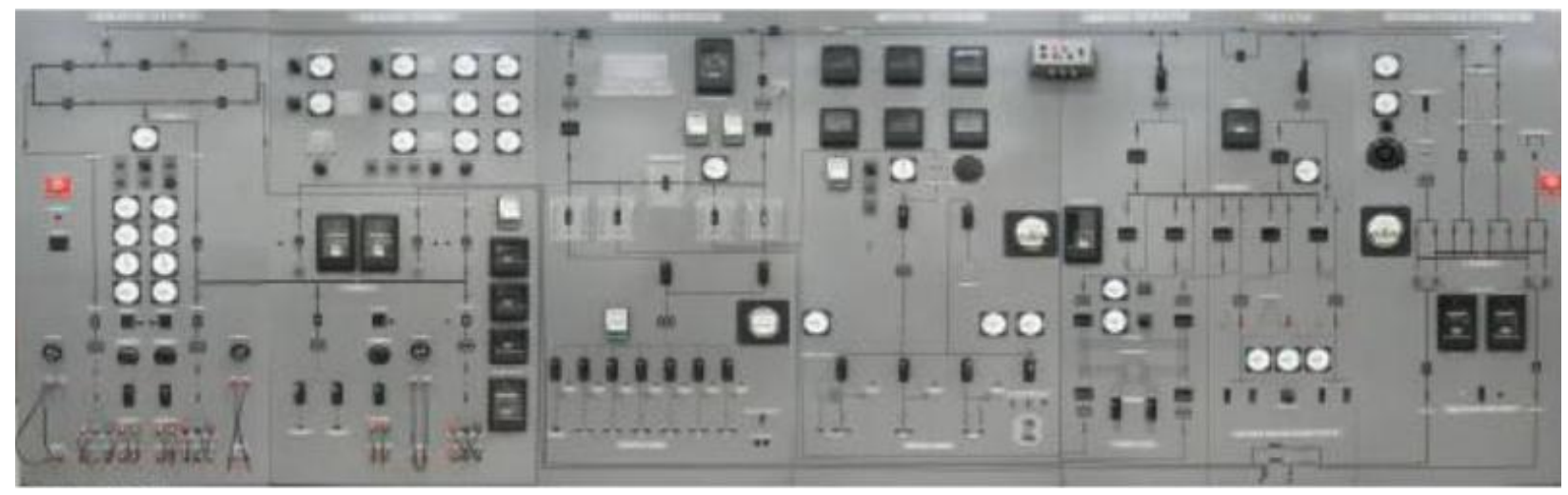

Figure 2: Power System Simulator in Original Configuration

\subsection{2 - Software}

There are many types of software used in a Smart-Grid implementation. This thesis will focus on a framework for a Multi-Agent Grid Management System, applications for embedded systems development, and the Human-Machine-Interface. The framework of the MGMS allows data sharing, automated reconfiguration, and host for all other Smart Grid algorithms such as optimal dispatch and load shedding. Applications for embedded systems create the link between logic (MAS) and the physical world, while the HMI conveys information and offer a handle for humans to manipulate device status and settings. Only a proper integration between these systems will deliver effective and practical control architecture.

The Multi-Agent System must be designed to operate in coherence with utility standard procedure and government regulation. Case studies from the WVSC project will provide a map of acceptable and desired behavior during epochs of contingency. The architecture must be well organized and deliberate in its intent; in any real world environment, all resources form constraints. Computing 
requirements and bandwidth must be minimized. The proposed architecture must be able to efficiently deliver critical information to required nodes while maintaining the highest level of reliability.

The MGMS will interface the simulator via Freescale M52259Demokit modules. The M52259Demokit is a versatile package with many on-chip peripherals including ADCs, timers, General Purpose Input/Output ports, and an array of communication ports. One of the greatest strengths of a Multi-Agent System is the ability to share information in a dynamic system. Using Freescale Codewarrior IDE, the modules can be trusted to convey critical information through alternative communication routes used in industry.

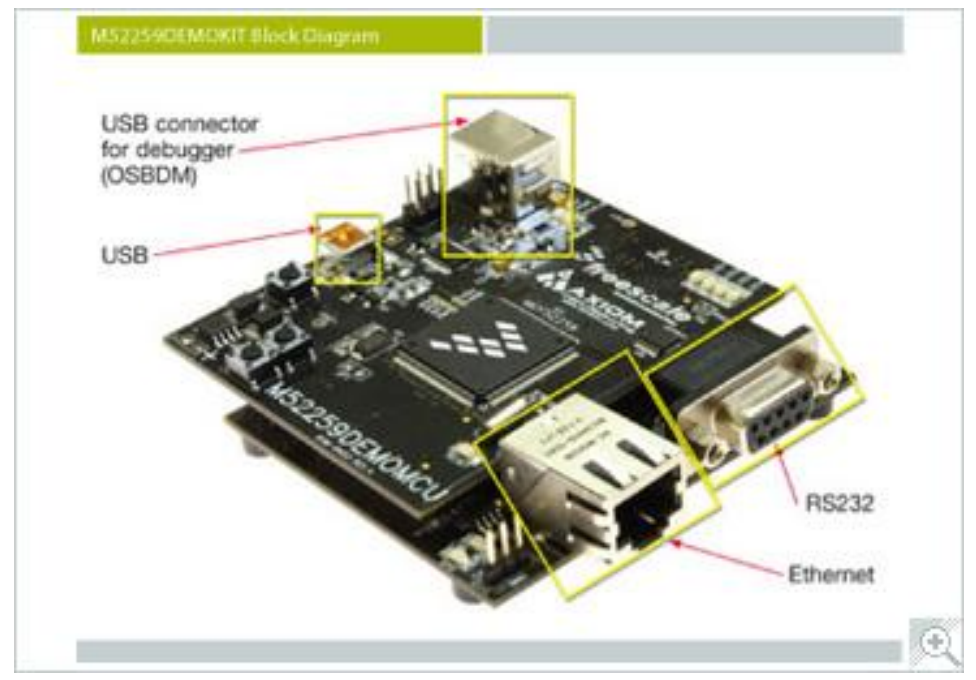

Figure 3: M52259DEMOKIT Module

Finally, The Human-Machine-Interface at the Power System Simulator will allow a user complete information access and control over the system. The combination of the SEL 3351 System Computing Platform along with SEL 5030 Quickset provides calibration, construction of information databases, event reports, and a wide range of functions designed to enable mastery of a distribution network's profile.

\subsection{3 - Summary}

Though the deliverables in the project are designed with a specific topology in mind, the approach maintains a scalable solution. The final hardware product will be able to support all technological endeavors, providing a functional test bed for decades into the future. The final software product will provide a versatile framework which can be applied to systems of any size with similar 
success. Together, the hardware and software designed in this project will maintain a fully featured test bed for Smart-Grid technologies.

\section{5 - Thesis Outline}

- Chapter Two will provide a literature review covering similar work in this field.

- Chapter Three will briefly explore the available SEL protection network including relay placement, HMI, and interface software.

- Chapter Four will discuss the Multi-Agent Grid Management system in detail,

- Sense and control module development in Chapter Five.

- Hardware integration results will be covered in Chapters Six.

- A Smart Grid application hosted on the Power System Simulator will be seen in Chapter Seven.

- Chapter Eight will draw conclusions from the work covered in this thesis. 


\section{Chapter Two - Literature Review}

Hardware simulation and MAS have been topics of great interest in the recent past. This chapter will present relevant research as pertaining to the Hardware Platform for MGMS. First, hybrid simulation and pure hardware simulation will be explored, with emphasis on previous work performed on the Power System Simulator. Next, MAS and their applications to power systems will be investigated. Historical approaches for MAS to power systems touched, followed by a direct application to the Power System Simulator.

\section{1 - Simulation}

\subsection{1 - Software Simulation}

The value of simulation cannot be overstated. Cost of infrastructure and expertise required to construct and maintain a full scale Smart Grid test bed is simply unfeasible to all but the wealthiest of entities. To remedy this fact, simulation software and companies enterprising in its use have become common, if not ubiquitous. Results gathered from evaluating system models provide valuable insight to operation. Indeed, software simulation is almost mandatory before any rational investment could be placed in a design.

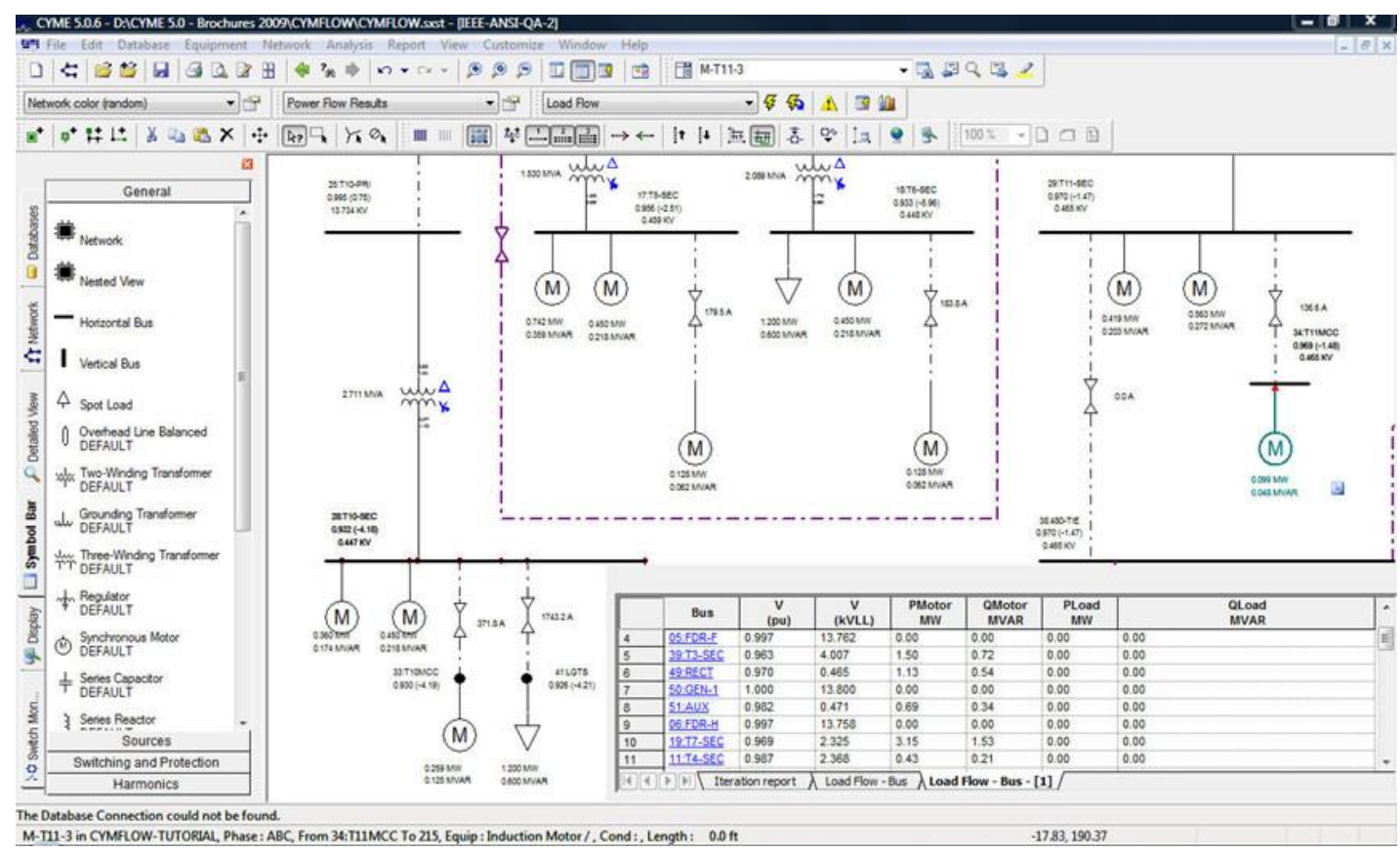

Figure 4: CYME GUI (CYME Power Engineering Software \& Solutions, 2011) 
Yet, while software simulation can yield a great deal of powerful knowledge, simulation fails to envelope the entire set of phenomenon which constitutes our physical world. Simply put, software simulation cannot yield results to the same accuracy which we can rely upon from a full implementation. Because of these shortcomings, hybrid and full hardware simulation platforms have become more popular as new control algorithms become more daring and complex. Hybrid systems provide a compromise between the cost of a physical system and the precision of a full hardware system. On the other hand, full hardware implementations provide exhibition of true natural phenomenon in all of its detail, but at a higher cost.

The next generation of software simulation is 'hardware in the loop' simulation. Several companies offer products designed to emulate hardware while interfacing physical systems. These systems are commonly used when testing advanced algorithms, evaluating protective schemes, testing interaction between electrical installations, and other situations which place infrastructure in jeopardy.

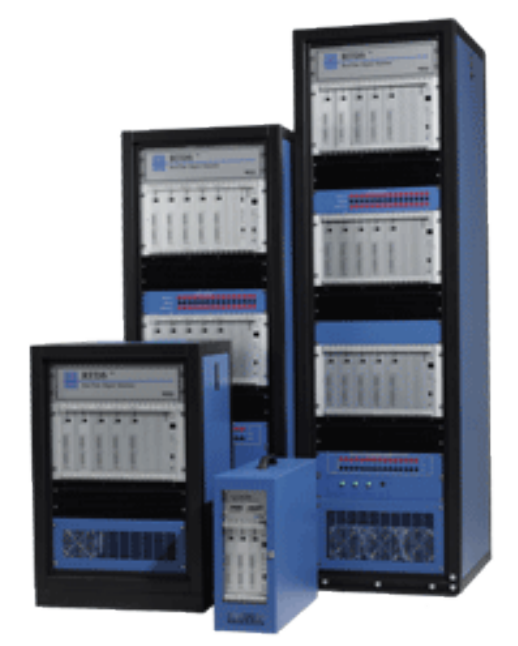

Figure 5: Real Time Digital Simulator Rack (RTDS Hardware, 2011)

The value and significance of such systems is that they have the computational ability to solve power system equations fast enough to provide responses to the physical devices connected to it. Within this emulated system, a user is able to design operating environments, modify conditions (while in 
simulation), and perform extensive data acquisition. Even with this flexibility, the components modeled within the system are only mathematical approximations, and cannot replicate the detail of nature. However, the efficiency at which systems can be configured and evaluated make hybrid simulation a powerful tool for deploying new technologies.

\subsection{2 - Hardware Simulation}

Humans have struggled to quantitatively describe our environment since the dawn of our existence. Despite the best of efforts, there is still an infinite amount of phenomenon waiting to be fully illuminated by the language of mathematics; all models created by man are incomplete. The moment that an ideal is taken practically is the moment details are eliminated from perspective. This is necessary, and surely welcome in many cases. Take, for instance, classical kinematics. We may neglect the moon's pull

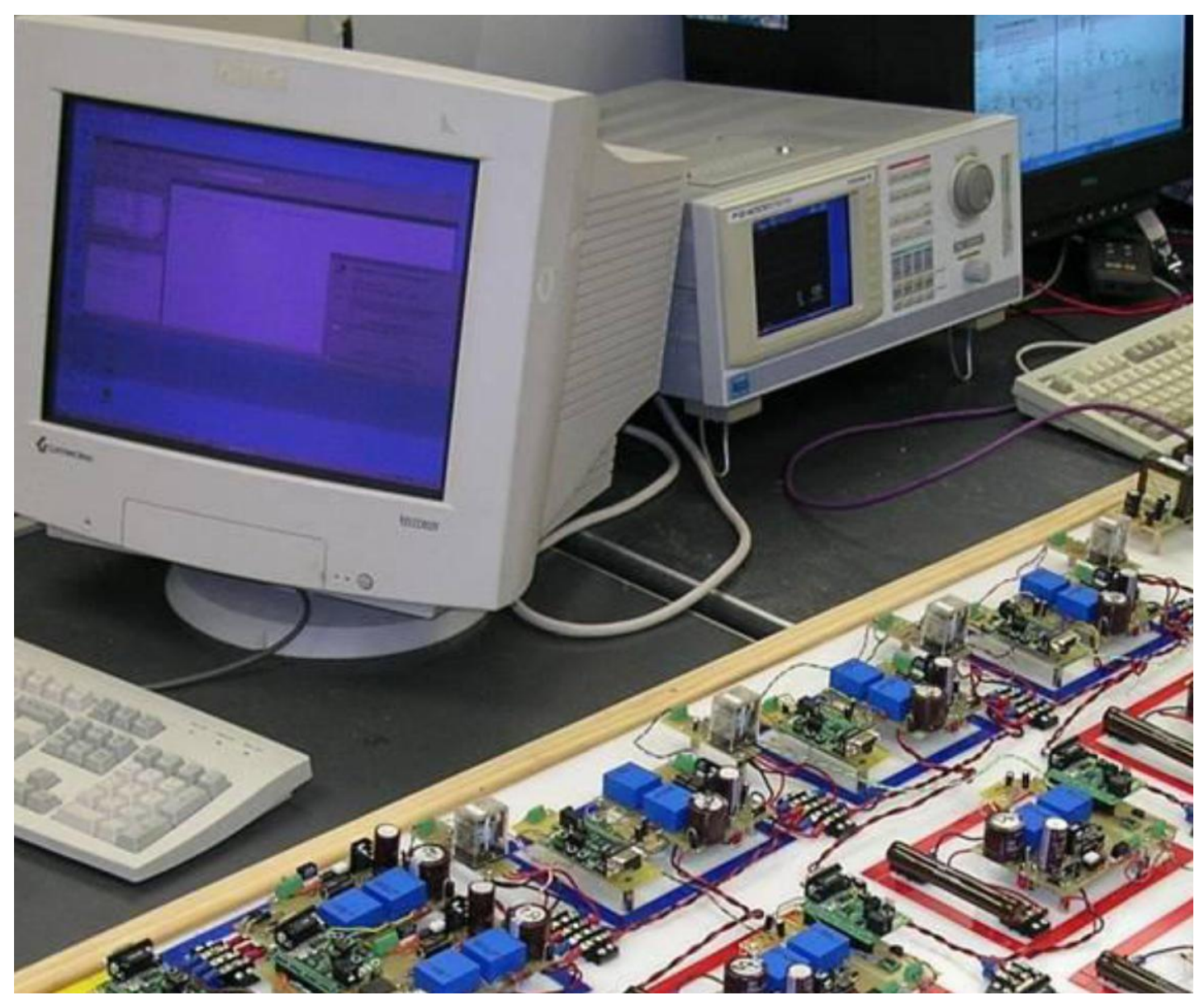

Figure 6: Hardware Simulation of Shipboard Power System

on a ball thrown from a train, the impact is negligible. However, there are many cases when 
oversimplification could pose a serious detriment. When the value of infrastructure and labor is high, the cost of negligence becomes high as well. A power system is one such case that oversimplification could result in regret.

Hardware test beds for distribution systems are able to account for much of the natural phenomenon which is too complex to model effectively, and can provide accurate responses at scaled levels. To this end, the Hampden Engineering Corporation Power System Simulator was purchased for educational and research purposes. As noted in Chapter 1, the Power System Simulator is a scaled replica of a distribution power system. The structure is designed to showcase all major facets of such a distribution level system, including residential and industrial loads, a substation, interconnection of DGs, and connection to local utility. Protective relays and breakers are distributed throughout the simulator at critical junctions which mimics industry practice. The Power System Simulator provides a qualitatively accurate rendition of a distribution system found in industry.

Though built in the late 70 's, the frame provides ample opportunity to be upgraded to portray a modern, and even futuristic, system. The core characteristics of a Smart Grid were identified and incorporated into the plan to upgrade the Power System Simulator to a test bed of the future. Former work within the APERC team, primarily Michael Spencer, was focused upon planning devices for the upgrade. This included new digital relays and sense and control modules. This thesis is the progression of his work, which will be covered in greater detail in later chapters.

\subsection{MAS in Power Systems}

The Multi-Agent System field is being researched extensively, as the general approach is applicable to many challenging problems. MAS's scalable, adaptable nature has given rise to many algorithms employed in power systems. Though this thesis maintains a high-level, qualitative approach to MAS, the reader should be aware of the various applications which would proceed from the completion of this project.

The most fundamental of algorithms for a power system is automated Fault Location, Isolation, and Reconfiguration (FLIR). Beyond this, MAS have been applied to regulate voltage, optimize protection coordination, manage DER's, and provide economical dispatch. When used within a MAS, these applications constitute a Multi-Agent Grid Management System (MGMS). 


\subsection{1 - FLIR}

The essential function of a MGMS is to protect the system from contingencies; all other functions are secondary to maintaining a stable, healthy system. Hence, the Fault Location, Isolation, and Reconfiguration algorithm takes highest priority of agent functions. There are many proposed solutions for an effective FLIR, with many different architecture and communication schemes. Chapter 4 will explore an algorithm designed with the WVSC project in mind, but also intended to be exported to other systems.

\subsection{3 - Optimization of Coordination}

Due to industry or company standards and regulation, a MGMS operating in a distribution system may be restrained until certain conditions occur, for instance, a recloser locking out. Because of this, standard protection logic (time curve coordination and other methods) will likely be a mandatory and critical aspect of protection.

Distributed Agents have been used to analyze the performance of a system in the event of a contingency. Using post-fault data, the agents are able to provide control for relay settings to improve reliability and selectivity performance. Supervisory actions are taken to ensure a fault is isolated with the minimum use of breakers, maximizing the service area after a fault.

\subsection{4 - Power Quality Regulation}

With the increased use of sensitive electric devices, power quality has become a high priority. Both frequency and voltage must be maintained within limits to avoid damage to electronics or electromechanical systems. Agents have been used to monitor and regulate these quantities, particularly in microgrids, where both generation and load can be managed. Power quality is a very relevant concern, and poses great interest in the MAS community. 


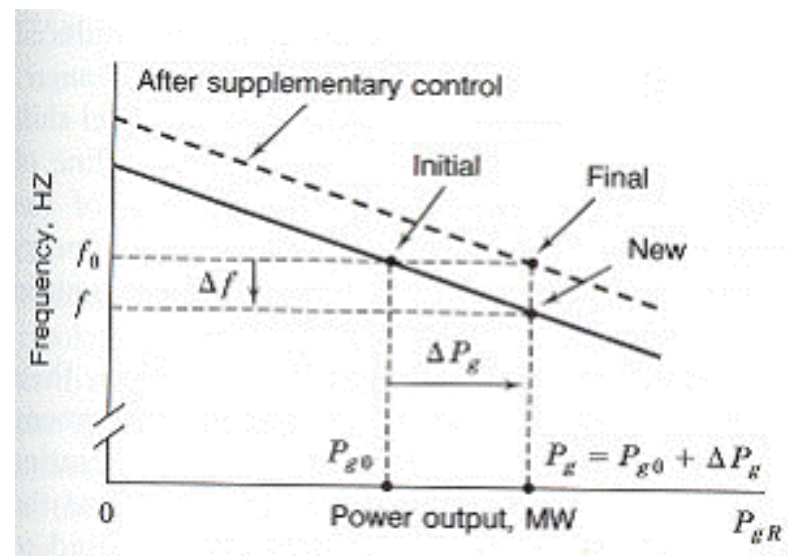

(b)

Figure 7: Frequency Vs. Power (Grainger, 1994)

\subsection{5 - DER Management}

With increasing demand on an aging infrastructure, many utilities are utilizing distributed generation to cut loading on power lines. Not limited to microgrids, distributed energy resource management may quickly become an essential part of a high percentage of distribution systems. DGs change the dynamics of a network in many ways. DGs complicate the system by removing the radial

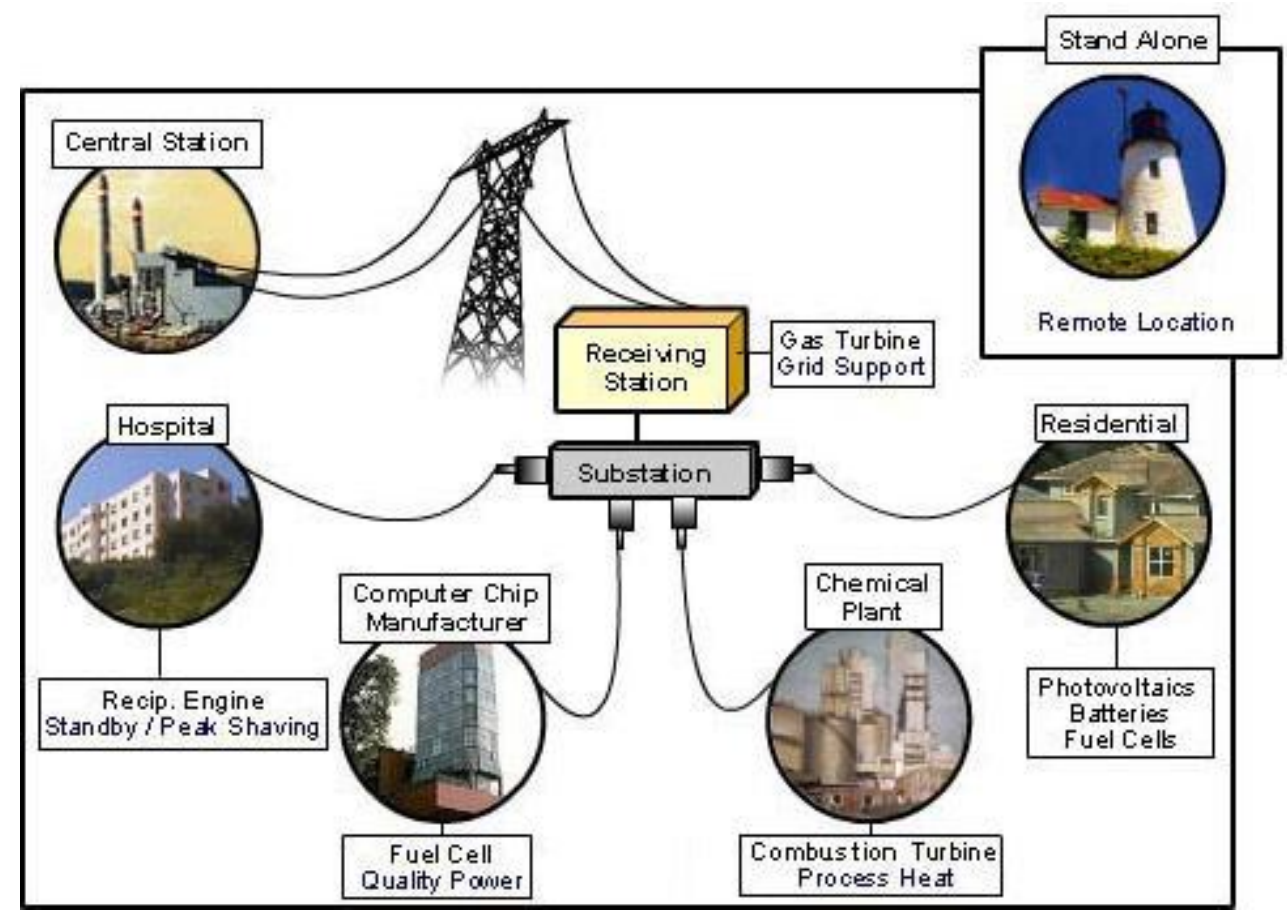

Figure 8: Commonly Used Distributed Energy Resources (Barney L. Capehart, 2010) 
topology, which requires added consideration to protection and additional hardware for switching. The decentralized nature of MAS makes them particularly suitable for such a task, and may prove to become the most cost effective and reliable method of integrating distributed energy resources into and antiquated power grid.

\subsection{6 - Economic Dispatch}

Some mutli-agent systems have been specialized to intercept market data and formulate strategies which a company can adopt. A MAS has the ability to react immediately to spot market changes and maximize savings on a transmission level. With increasing penetration of DERs, economic dispatch is a problem no longer limited to transmission levels. In tandem with DER Management, a MAS could have the ability to optimally dispatch power throughout a distribution system, conserving resources for all parties involved

\subsection{7 - Consolidation}

With the range of functions which a MAS can accommodate, it takes little intuition to imagine a power system under the complete supervision of agents. When performing one or more of the responsibilities described above, the MAS can be recognized as a Multi-Agent Grid Management System. Though individual algorithms have been developed and proven, an architecture which hosts all of these

functions and facilitates their cooperation and coordination is still a hurdle to be leapt. This thesis will propose a framework intended to do such a task. 


\section{3 - MAS Applied to the Power System Simulator}

A recent graduate of the APERC team, Dr. Rabie Belkacemi, focused much of his work on a FLIR algorithm which was tested on the Power System Simulator. Using the Java Agent Development Environment (JADE), Dr. Belkacemi designed an agent algorithm hosted on Freescale MCU's which manipulates the hardware of the simulator to protect and restore power to loads in the event of a fault.

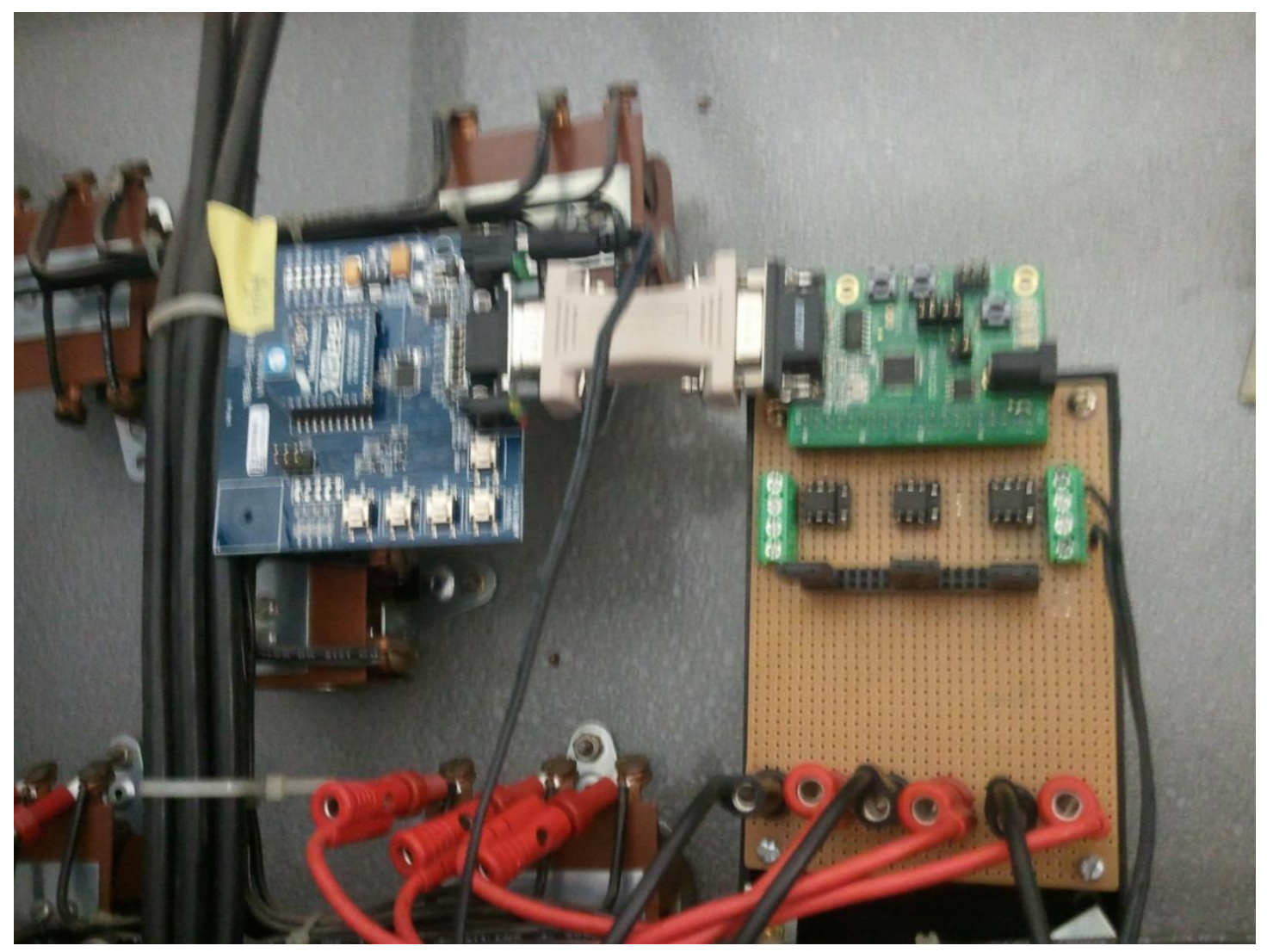

Figure 9: Dr. Belkacemi's Agent Host \& Switch

The system is designed to maintain a load in the residential section of the simulator. Power is initially fed through the top feeder, followed by a step down transformer and breakers. A fault is simulated by applying a large load to the line. When this occurs, the agents monitoring the main feeder identify a threshold violation (Location stage)and break the current flowing from the feeder (Isolation stage), which removes the fault from the main system. The agent on the second feeder then initiates its switch and reroutes power to the load, effectively restoring power to the load within fractions of a second. 


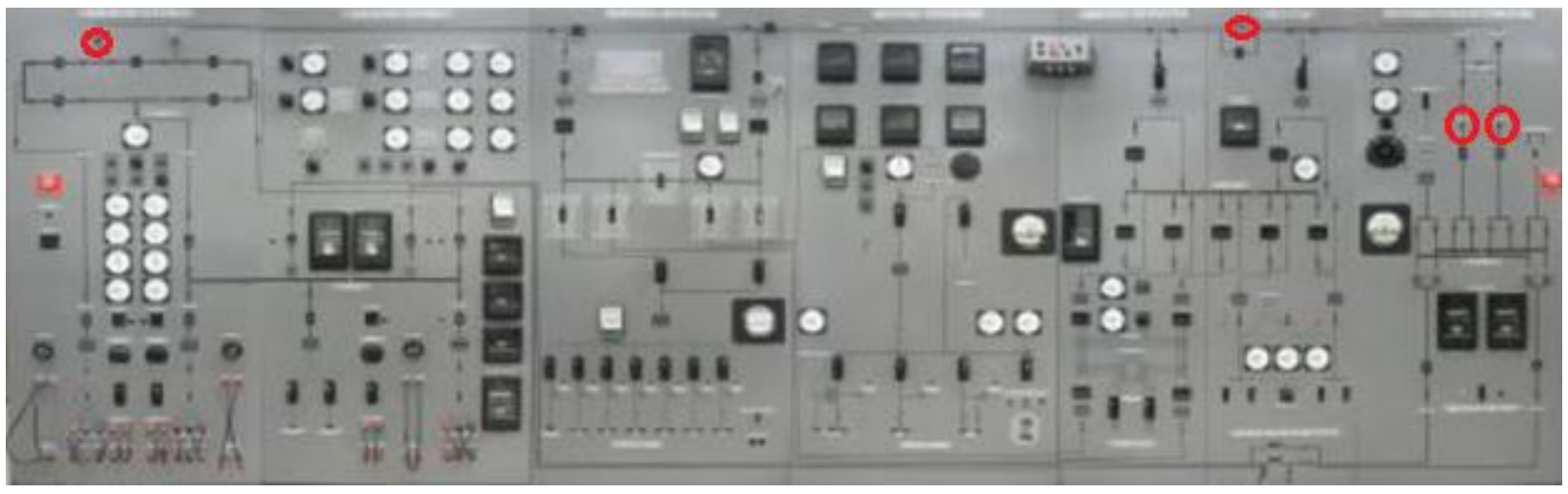

Figure 10: Relay Locations on Simulator

Though the scenario is simple, it showcases an MAS's ability to respond to a contingency intelligently and immediately. The demonstration presents functionality which will eventually be implemented system wide on the Power System Simulator. 


\section{Chapter Three - SEL Protection Network}

\section{1 - Protection System Hardware for Distribution Networks}

As power systems grow in complexity, the protective network used to protect the structure must grow as well. Smart Grid applications mandate intelligent devices capable of delivering safer, more reliable, and more economical solutions to classical protective issues. SEL provides a line of products intended to satisfy these needs, ranging from generator and transmission protection to distribution automation and control systems.

When designing a robust protective system for use in a distribution system, one must consider all possible points of failure. Feeder lines, transformers, loads, and generators all pose potential contingency points. Implementing the appropriate data acquisition and processing allows for fast identification and mitigation of faults or other types of failures. SEL has designed relays suitable for each of these scenarios. The relays include the necessary ports for data acquisition and communication to ensure that the system operator has complete knowledge of the system. This chapter will explore the protective systems available from SEL which are applicable to the Power System Simulator.

\section{2 - Relays}

The Power System Simulator was designed with an integrated electromechanical relay protection network. These relays are placed in critical areas, mimicking industry use. Though effective in their original purpose, these antiquated devices are unable to provide the functionality necessary for a MultiAgent Grid Management System. The electromechanical relays have been paralleled with their digital counterparts which were donated by SEL. This will allow for data acquisition and a more flexible and accessible coordination structure.

\subsection{1 - Overcurrent and Directional}

Being the simplest and most obvious indication of a fault, the most basic task in protection is overcurrent detection. The overcurrent relay is surely a staple of distribution protection, with many optional features and capabilities.

\subsubsection{1 - SEL 351 and SEL- $351 S$}

The 351 is the most basic of overcurrent relays provided by SEL. The package includes built-in synchrophasors, an array of communication schemes, and power quality metering. As with most SEL relays, the device is ready to accept input from current- and potential- transformers on all phases. Front 
mounted pushbuttons make manual clearing simple. Reclosing control with programmable four-shot breaker utilizing synchronism and voltage check logic is included. Phase sequence (positive, negative, zero), overcurrent time curves, and frequency elements are all available to provide complete and flexible protection.

SEL-351

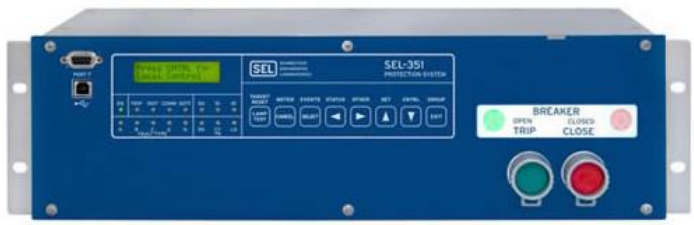

\section{SEL-351S}

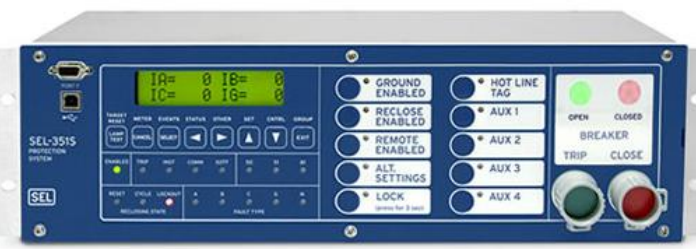

Figure 11: SEL 351 and 351S Overcurrent Protection Relays (Schweitzer Engineering Laboratories, 2011)

The $351 \mathrm{~S}$ provides additional functionality and convenience over the 351 . The $351 \mathrm{~S}$ includes an impedance-based fault locator for accurate distance to fault measurements. Thirty eight traditional recloser curves can be used to coordinate with downstream circuit reclosers. An optional fiber-optic communication port for secure and noise-free transmission of data can be implemented. Finally, the 351S offers the expanded operator control array found on the front of the device.

\subsubsection{2 - SEL 751A}

The SEL-751A is designed for industrial feeder protection. Flexible I/O options are available, with easy installation for convenient access. The system also delivers a range of features and functions to ensure a feeder is operating completely within specification.

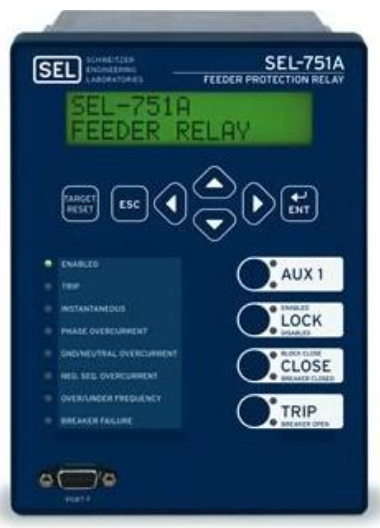

Figure 12: SEL-751A (Schweitzer Engineering Laboratories, 2011) 
Fiber optic standard, RS-232 or RS-485and Ethernet are available for integration with existing infrastructure, and can immediately alert personnel with proprietary software. SELogic Control Equations use math and logic combinations of sampled values for customized applications.

The device allows for a flexible control scheme with time- or instantaneous- overcurrent, voltage sag and swell violation, and frequency elements. This includes frequency deviation, synchronism, DC supply check, arc-flash detection, and demand metering elements. The four-shot recloser with optional synchronism can be used for clearing of temporary faults.

\subsection{2 - Current Differential}

The transformer is critical component of AC power systems. By increasing the voltage magnitude, the current magnitude can be decreased, allowing the use of smaller conductors. This requires high voltages to be contained in relatively small areas and for the transformer itself to handle a large amount of current on the low voltage side. This combination yields a hazardous environment. With extended use or unintended operating conditions, transformers often experience failure of insulation, causing internal faults. Current differential relays typically monitor the current entering and leaving a transformer. By comparing these values, the relay is able to detect a fault within the transformer itself.

\section{SEL-387A}

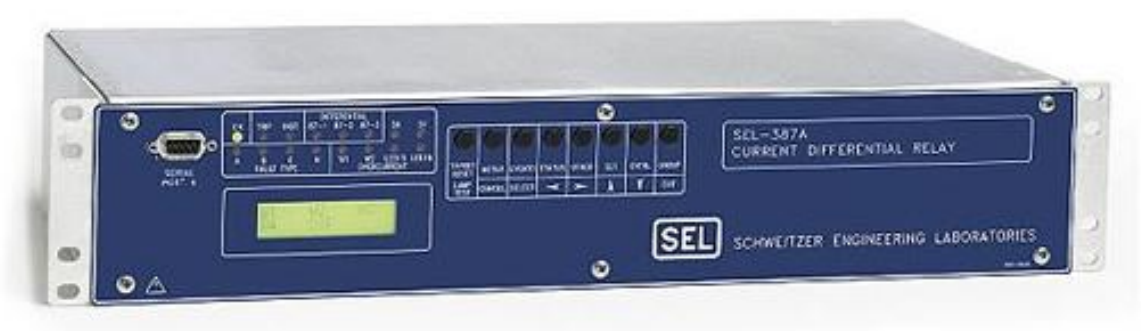

Figure 13: SEL-387A (Schweitzer Engineering Laboratories, 2011)

The SEL-387A Current Differential Relay is designed to be a flexible and effective solution to transformer protections. Current differential protection with harmonic blocking/restraint elements and unrestrained differential elements provide fast operation for high-magnitude internal faults. Comprehensive protection for individual windings is offered with torque-controllable overcurrent 
elements including instantaneous and inverse time elements per phase, negative sequence, and residual ground currents.

A robust control set is also integrated to the SEL-387A. Restricted Earth Fault (REF) logic with SELogic control equations, timers, and remote control elements are used for advanced protection and control schemes. Oscillographic event reports deliver 60 cycles of detailed waveform data.

\subsection{3 - Revenue Meter}

In Smart Grid applications are synonymous with energy management. Considering this, it is crucial to the effectiveness of the system that loads are extensively monitored for power quality and energy usage. Furthermore, efficient operation mandates that the monitoring device must be able to integrate seamlessly with the rest of the control architecture.

To this end, Schweitzer offers the SEL-734P Power Quality Meter. Advertised as 'the perfect tool for power quality (PQ) analysis and data recording,' the 734P monitors nearly all conceivable PQ quantities. Harmonics up to the $50^{\text {th }}$ order, flicker measurement, synchrophasors, load profile recorders, 8 $\mathrm{kHz}$ waveform analysis, predictive demand, frequency recording, and automated event recording retrieval are all functionalities held within the device.

\section{Legacy SEL-734P Power Quality Meter}

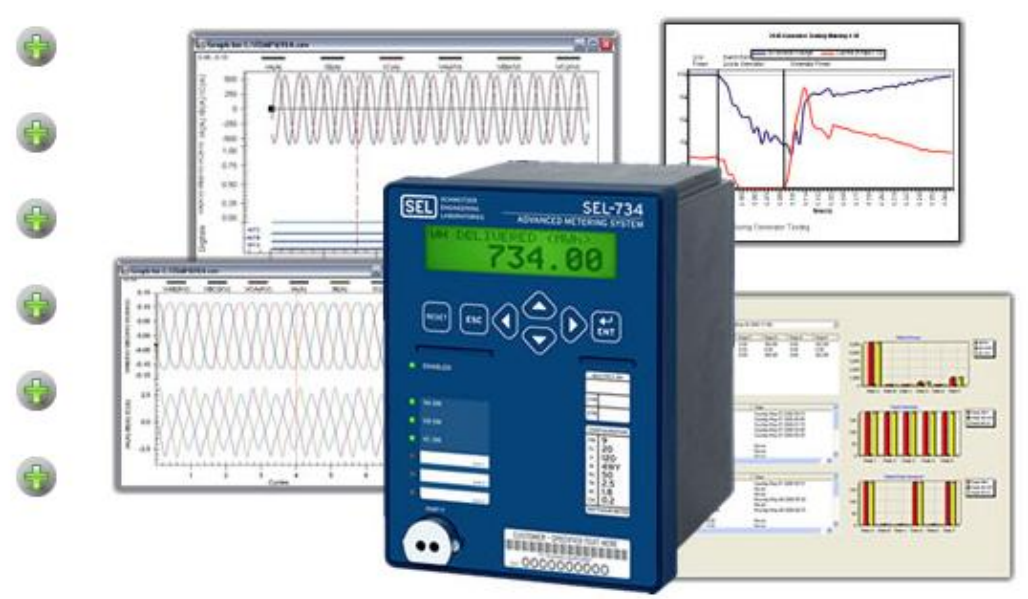

Figure 14: SEL-734P (Schweitzer Engineering Laboratories, 2011)

The system is also designed with integration in mind with seven communication ports and eight protocols including 10/100BASE-T or 100BASE-FX Ethernet, DNP3, RS-232 and RS-485. Fiber optic adapter for serial communications provide a fast and reliable solution to noisy environments. 120/240 VAC or 12/24 VDC inputs are conveniently available to power the device. 


\subsection{4 - Generator}

Distributed generation is a practice which places generation near load centers in order to cut the load carried from substation feeders. Such implementations can postpone or completely eliminate the upsizing of substations and feeder lines. Further applications include micro-grid operation, where islands of loads can be powered independently of the main power grid.

Although the concept is financially promising, the practice brings forth a great deal of obstacles to an already complex system. Distributed generation destroys the radial network, adding challenges to protection coordination and conventional logic. Energy management under island mode is critical to maintain a stable system, and reconnection to the main grid must eventually be achieved. To overcome these difficulties, it is necessary to monitor a DG thoroughly and provide adequate control for the necessary variables.

The SEL-300G is designed to provide unsurpassed protection, monitoring, and control of electric generators. Voltage, current, and frequency elements, ground fault protection, current differential, loss of synchronism, over excitation, and reverse power are all modules included in the package. Event reporting and high accuracy metering reduce external metering requirements, and the standard communication set offered by SEL makes integration simple

\section{SEL-300G}

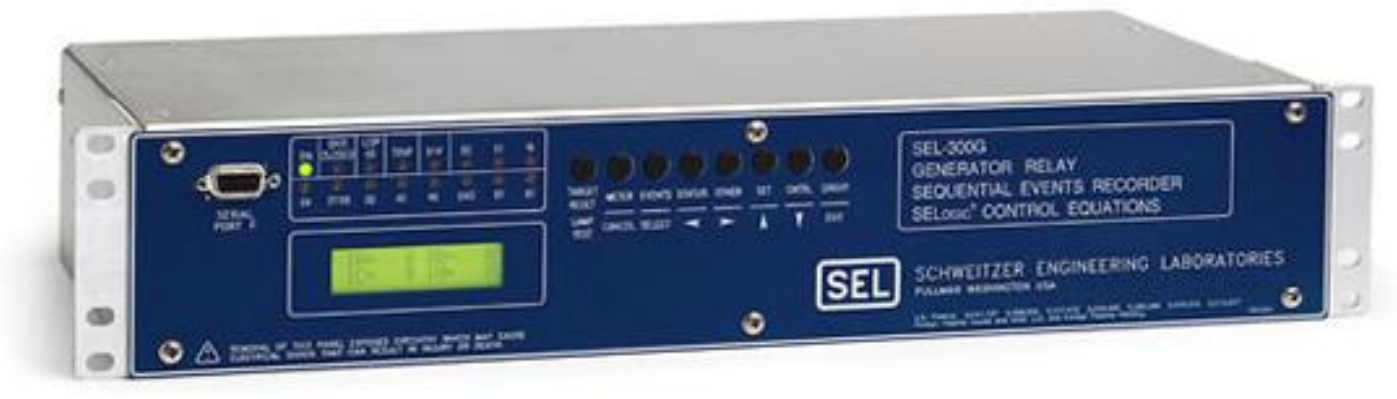

\section{3 - Human Machine Interface}

Relays perform critical monitoring and protection functions which can involve complex mathematical or logical sequencing. However, without an operator's ability to interface with the relays in a user-friendly and concise method, the information gathered by the relays is practically inaccessible. Making changes to relay settings could be difficult or even dangerous. Without an effective 
communication system, the information and functionalities offered by the relays are squandered. It is crucial that an area's protective system is networked to provide a access to data and relay parameters.

With this in mind, SEL designed the 3351 System Computing Platform. The 3351 is a Windowsbased ruggedized computer with the communications and interface software necessary to achieve a complete overview of a power system. The system contains no moving parts, which increases reliability in hazardous environment. Fiber optics, Ethernet, and a 16 port RS-232 and RS-485 hub are provided to interface the aforementioned relays. IRIG-B is included with the serial communications to ensure synchronized measurements. A Watchdog microcontroller provides an extra level of computer system reliability, rebooting the computer if there is a problem with the operating system. The device itself can be mounted in the vicinity of the relays if convenient, as Remote Desktop Connection or monitor, mouse, and keyboard can be used at a distance from the machine.

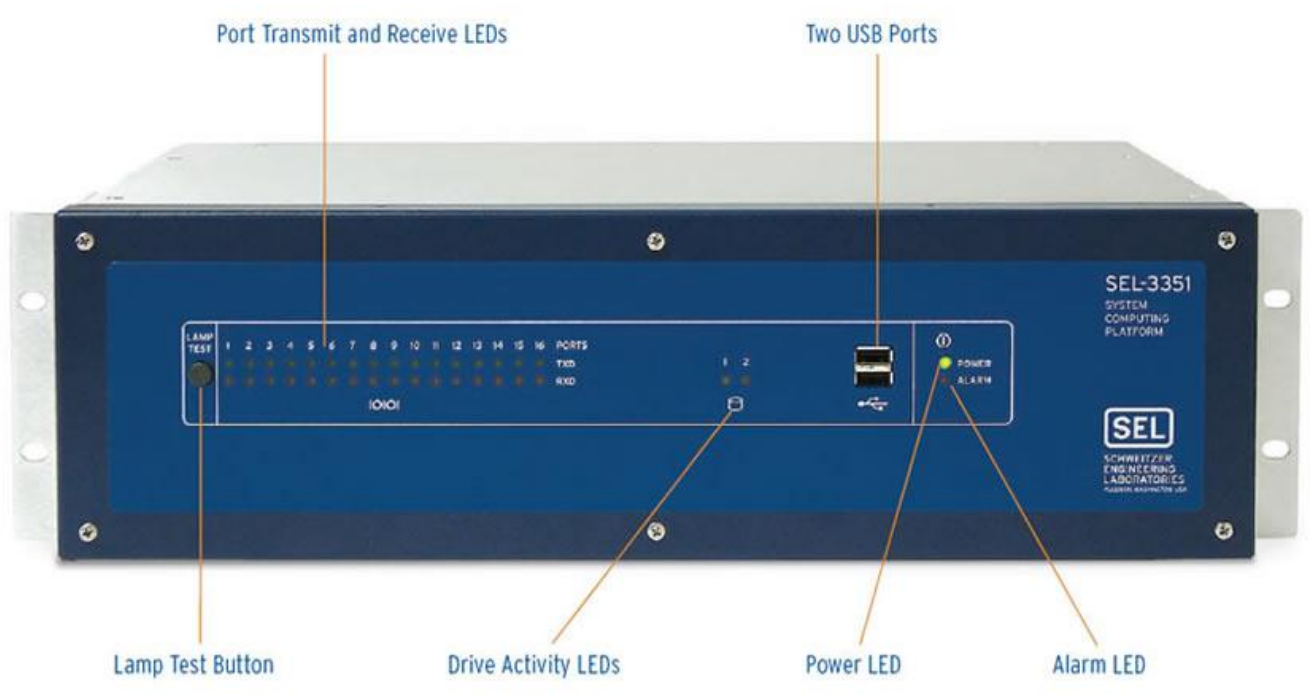

Figure 15: SEL 3351 (Schweitzer Engineering Laboratories, 2011) 


\section{Chapter Four - Multi-Agent Grid Management System}

\section{1 - Objectives of MGMS}

The fundamental goal of a MGMS is to manage energy within a power system. As discussed in Chapter Two, there are many methods which can be used to achieve this goal. Yet the management system drives for efficiency and reliability, a clear priority of its functions must be established if it is to deal with serious threats effectively. This thesis will focus on a framework for a MGMS which is structured to be decisive and stable as well as an algorithm to maximize the probability of survival during a contingency.

\section{2 - Architecture}

The architecture for the MGMS is designed arbitrarily so that it may be effective for energy management in all distribution networks. Although this is true, great resource is taken from the WV Super Circuit project; the WVSC not only encompasses all of the major facets of a Smart Grid, but it is also a true industry setting. Many practical considerations can be observed during collaboration with trade experts, which expedites deployment. The concept of exportability was paramount throughout the entire project: if the final products cannot be used in industry, the approach is trivial. Hence, the WVSC provides the working model for the development of a generic, exportable MGMS.

With this in mind, the WVSC topology and hardware capabilities were analyzed for approach. As stated in Chapter One, the network consists of several interconnected zones which are supplied by two substations. Where feeders pass through zones, switches have been installed to provide reconfiguration capability. Each of these switch locations will have sensors which provide RMS voltage and current data (at the minimum). Coupled with industry standard protocol for behavior under contingency this leads to the formulation of a hierarchical structure for the MAS. 


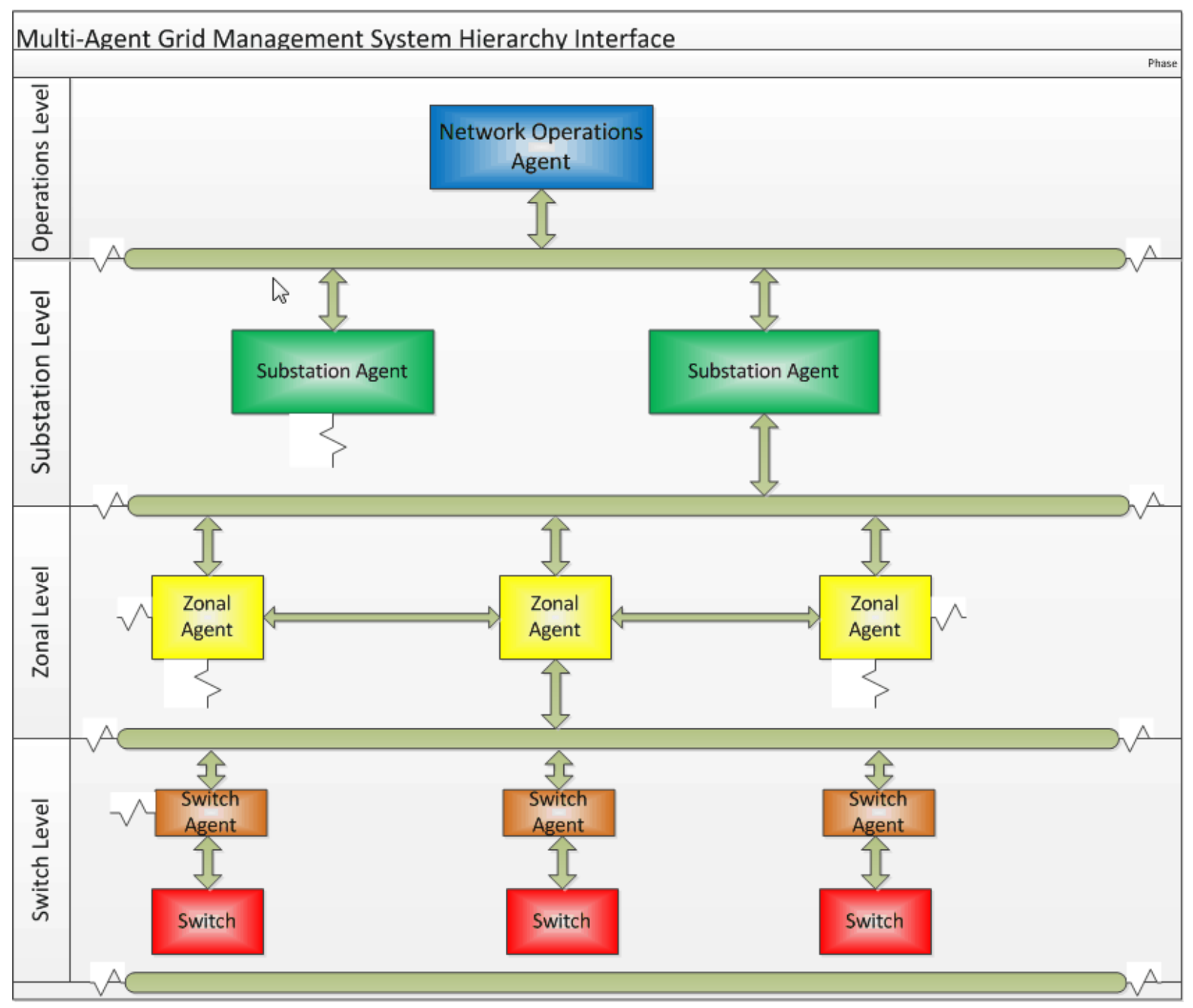

Figure 16: MGMS Hierarchy

At the lowest level of the hierarchy is the Switch Agent (SwA). The SwA is the primary interface with the physical world, performing switching operations and acquiring data. The SwAs receive directives through Zonal Agents (ZoA). The ZoAs are the building blocks of the entire system; they concentrate data of the physical system, interpret commands from higher levels, host algorithms, and issue commands to the SwAs.

The next level up from the ZoAs is the Substation Agent (SuA). The SuA monitors RTU and recloser status in the vicinity of the substation, as well as gathers aggregated loading and status information from the ZoAs for transfer upstream to the HMI. The SuA is the stepping stone from the network operator to the ZoAs. 
At the highest level of the hierarchy is the Network Operations Agent (NoA). The NoA establishes the link between humans and the MAS. This agent collects critical system information from SuAs and provides HMI to the Network Operator and interprets directives managing hardware.

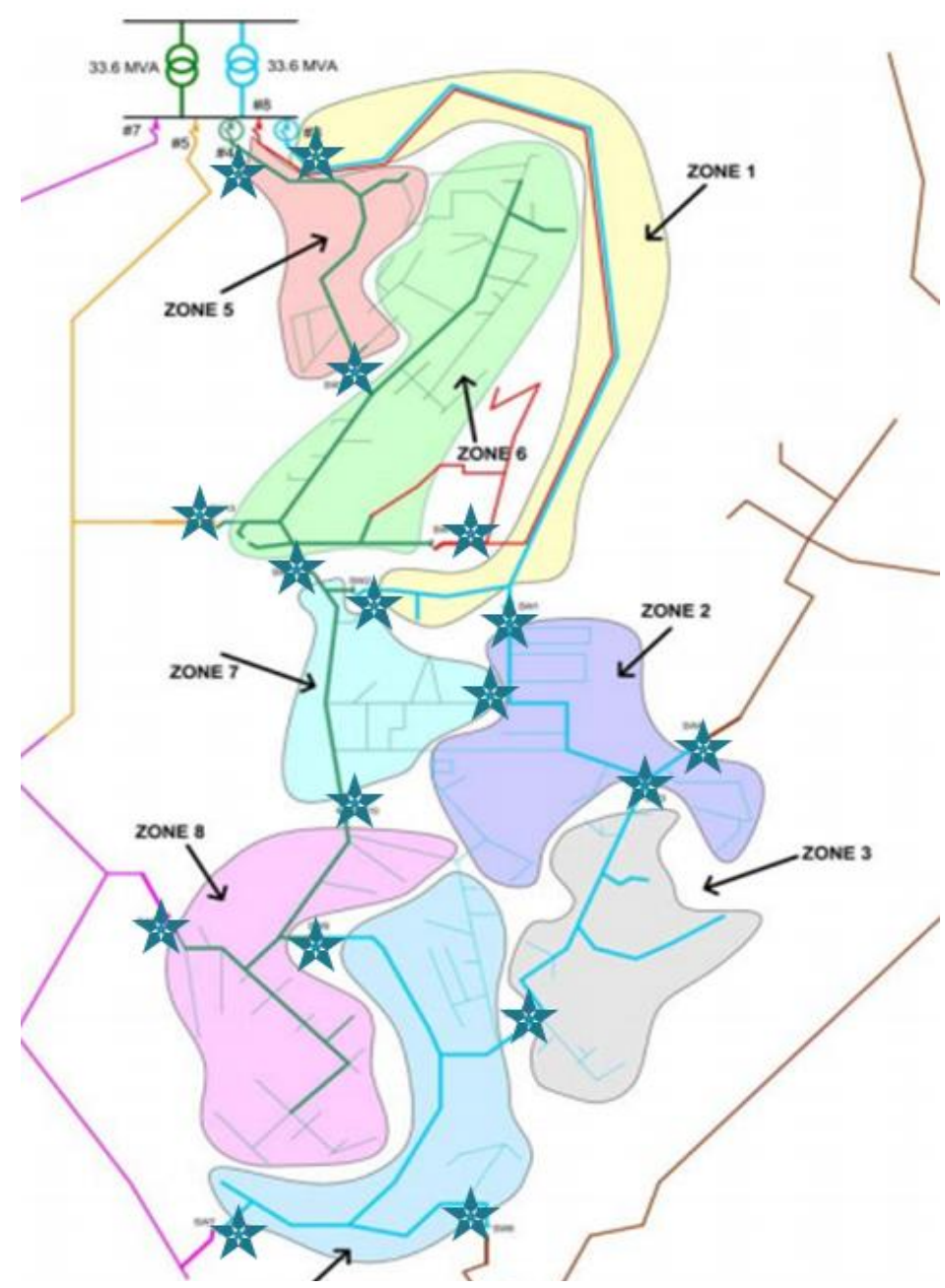

Figure 17: Switch Agents are Placed at Inter-Zone Ties

This scheme can be applied to any system with built- in reconfiguration capability. While maintaining a sense of generality, the framework defines a critical function set which can be used to manage the system while under contingency or during optimization procedures.

\section{3 - Fault Location Isolation \& Reconfiguration}

As a critical survival function, the FLIR is the highest priority of a MGMS. In the event of a fault, the FLIR will take precedence over all other algorithms, placing the grid into 'recovery' mode. The MAS will then determine the steps necessary to isolate the fault, and then reconfigure the system to maximize 
the service area. The utility may then manually clear the fault. Once this is complete, the system can be restored to its original configuration.

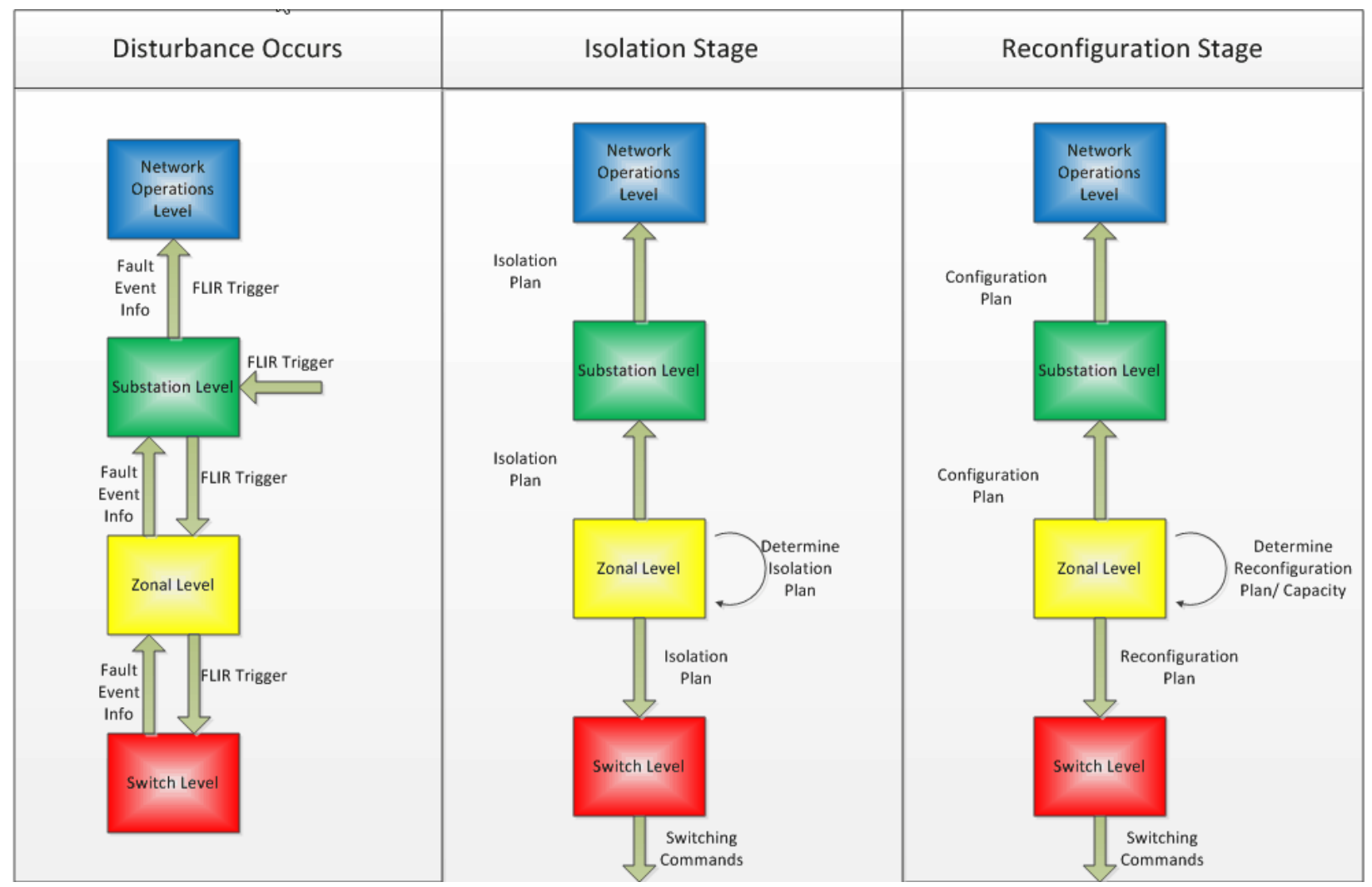

Figure 18: MGMS FLIR Flow Diagram

Initially, the MAS will be unable to act upon the system until the substation recloser locks out, as mandated by industry requirements. The FLIR trigger is sent from the recloser, and then distributed from the SuA to all other levels. The SwA sends data from the fault event upstream for the isolation plan and other algorithms.

Next, the Zonal Agents determine the procedure to isolate the fault and send the orders to the switch agents. The isolation plan is also sent to the NoA so that the network operator is aware of these changes. As the isolation is completed, the ZoAs immediately begin to determine the reconfiguration plan to maximize the service area. This includes ensuring that line limits are not violated which may further complicate the scenario. Prior to the restructuring, the plan must be verified by the network operator. As this is completed, the reconfiguration plan is then issued to the switch agents and the system will maintain this configuration until the utility can manually clear the fault. 
Though this sequence may sound simple, each agent has multiple responsibilities throughout the entire process. To illustrate this concept, the agent interaction chart was developed and can be seen on the following pages.

\section{4 - Further Work}

Though what has been discussed defines the framework and most critical function of a MGMS, there are still many facets which must be investigated and developed in order to provide a fully functioning system. Many of the applications discussed in Chapter 2 must be explored to deliver a robust energy management system. Also, exporting these concepts to hardware is a challenge which is a project entirely in its own.

A great challenge will be manifesting these concepts using agent coding software. JADE has been used for other applications to the Power System Simulator, while ZEUS Agent Building Toolkit is a software package providing a more user friendly GUI interface which reduces learning curves.

A MAS system employed for a MGMS is a great endeavor to partake. Developing the many functions and interfacing with industry standard devices pose a challenge. Despite this, the rewards of a fully functioning system are limitless, as is the potential for new, unexplored applications are more than enough to stimulate continued research in the area. 


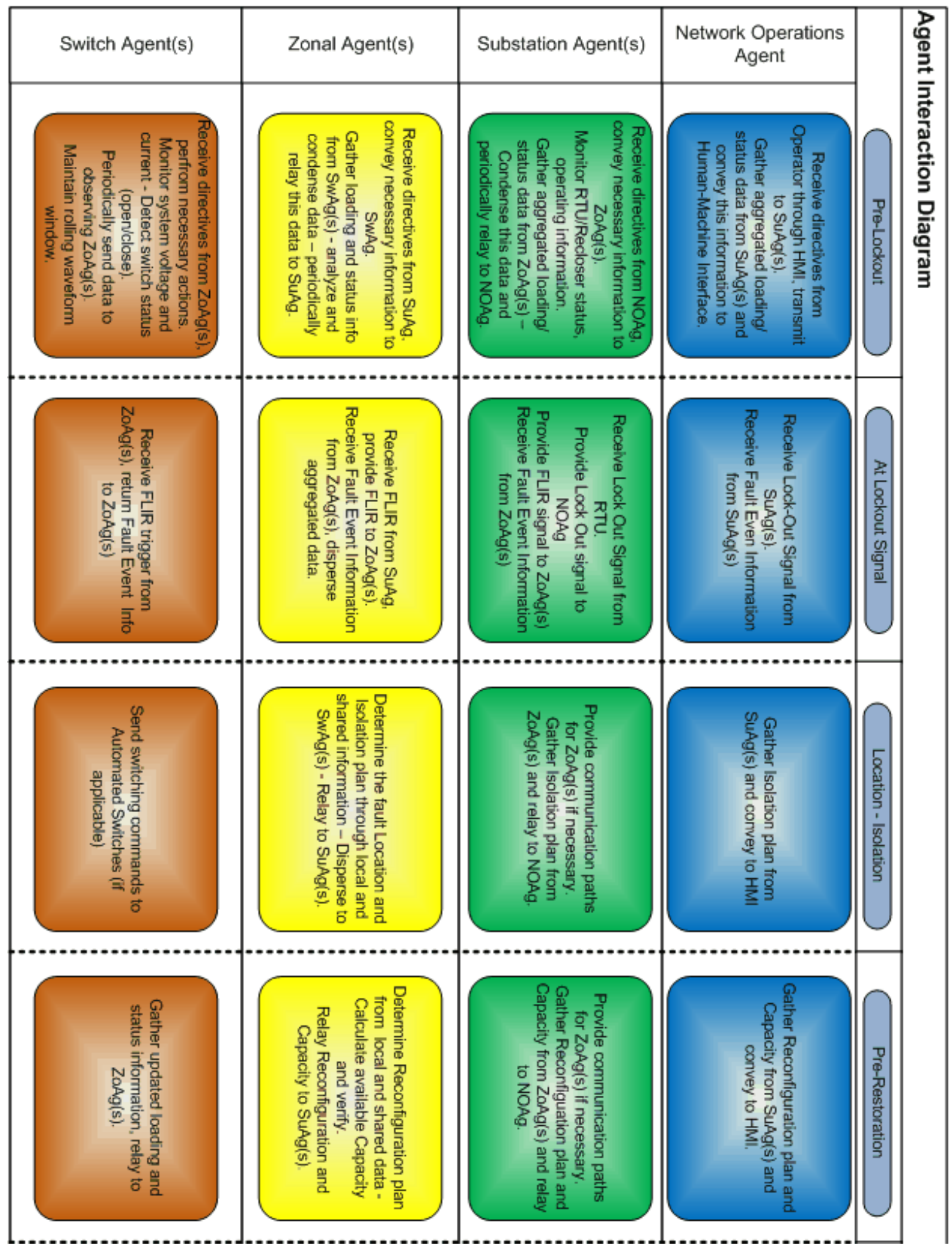




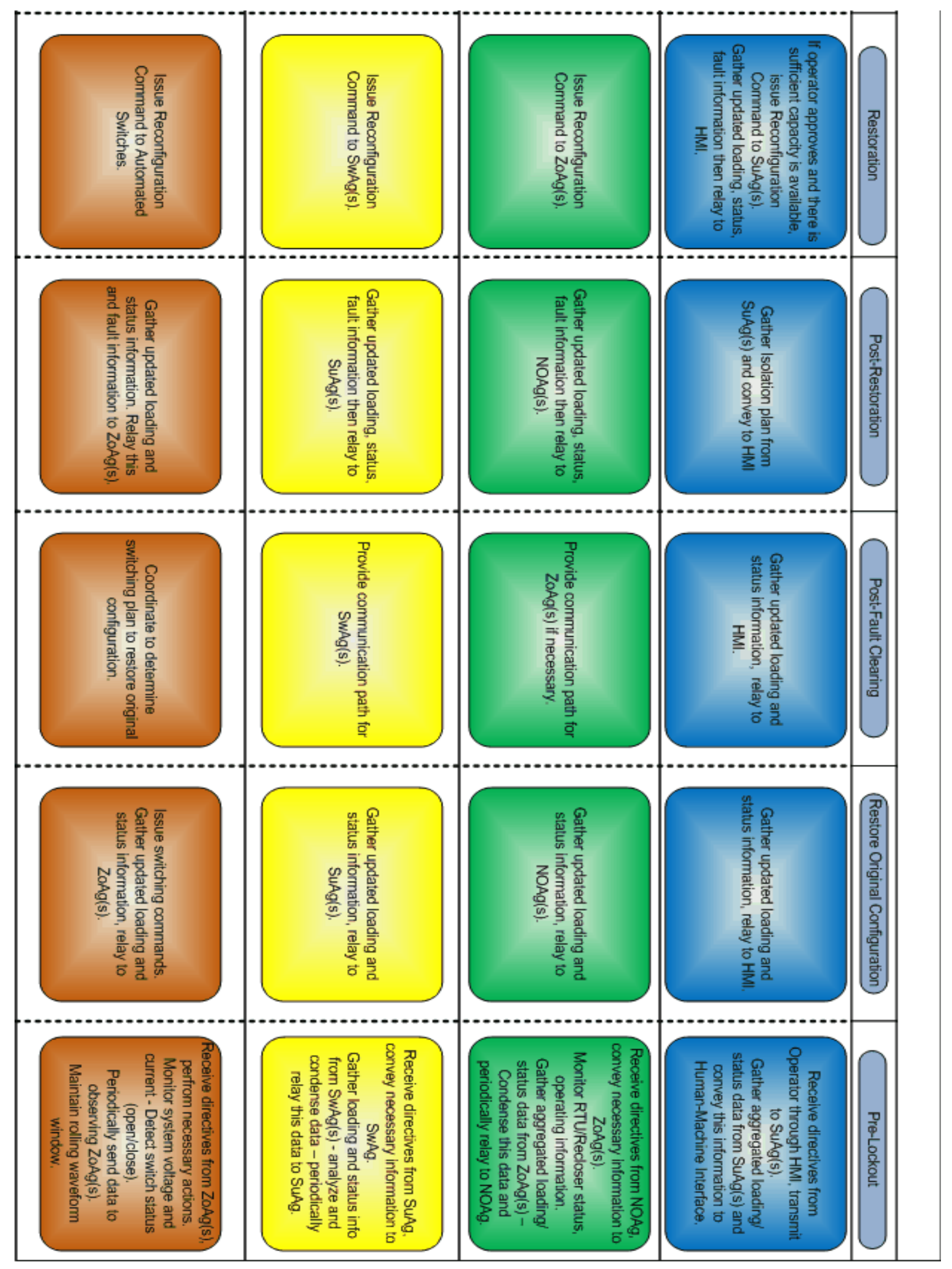

Figure 19: Agent Interaction Diagram Under FLIR 


\section{Chapter Five - Freescale Codewarrior Studio and Applications}

\subsection{Freescale Codewarrior Integrated Development Environment}

Freescale CodeWarrior is an Integrated Development Environment to design, configure, optimize, and export applications hosted on Freescale brand embedded systems. CodeWarrior provides a wide range of services which expedite the generation of code. An internal compiler and real time debugging provide a versatile and effective solution for the validation of projects. Coding can be done in $\mathrm{C}, \mathrm{C}++$, and assembly code. CodeWarrior version 7.1.2 was used for the development of the M52259Demokit.

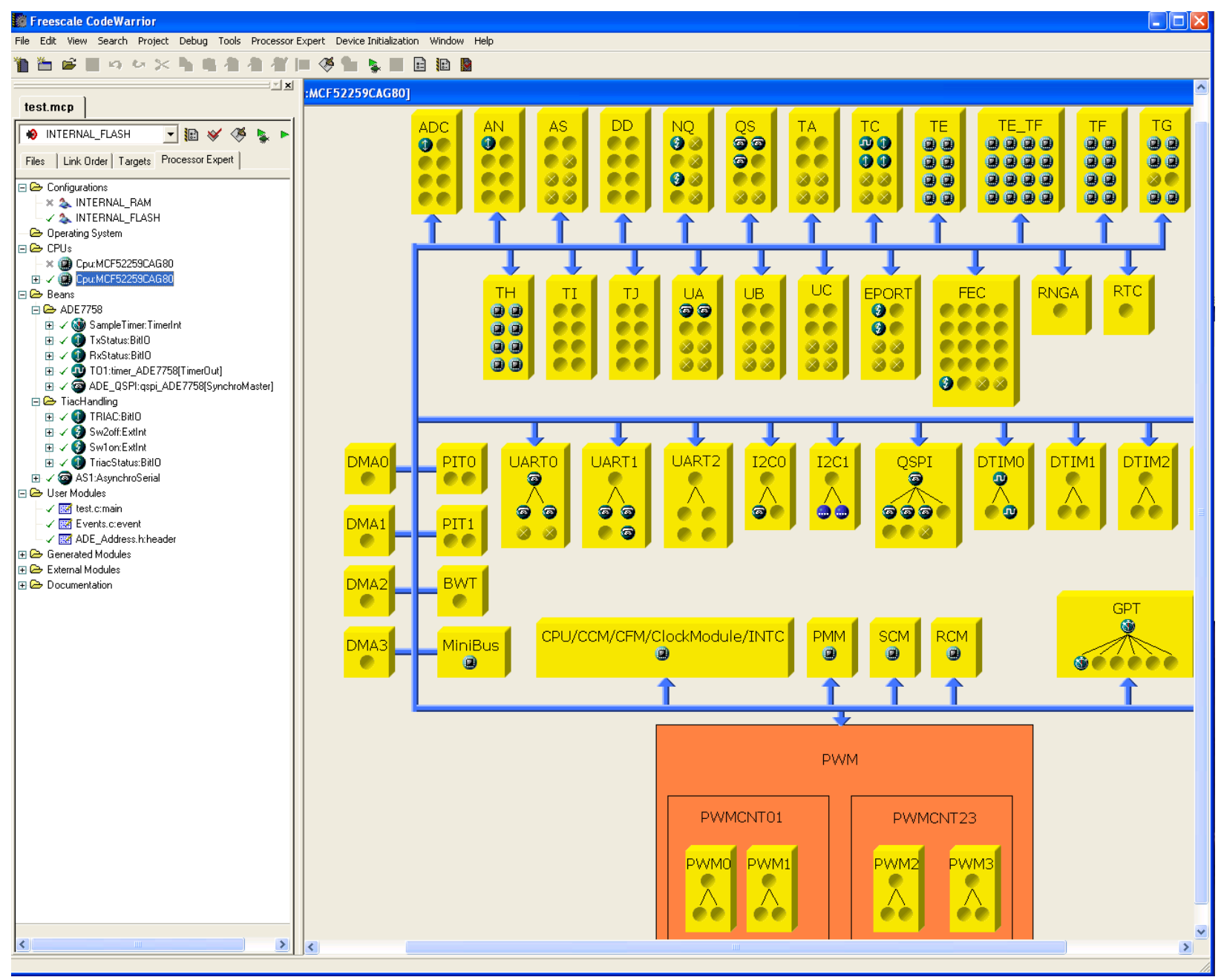

Figure 20: Freescale CodeWarrior GUI Snapshot 
CodeWarrior features an extensive GUI system to streamline the design procedure. As seen in the CodeWarrior figure, the GUI offers a overview of the entire project. Users have access to the chose the MCU for the project and the programming target (storage), add and manage hardware wizards called 'beans,' define user modules of code, and view modules of code generated by the program itself.

When starting a project, the user must define which MCU and development board they will be using in the project. This gives access to hardware peripherals and capabilities held by each MCU. In this project, the M52259Demokit board is used, which utilizes the MCF52259CAG80 processor. Other options include $\mathrm{C}$ and/or $\mathrm{C}++$ language sets, rapid application development options, full or minimal hardware support, and optimization options. Rapid application development consists of a set of tools to initialize and control hardware components.

Processor Expert Software is a subset of the CodeWarrior Rapid Application Development tool suite intended to generate source code for the manipulation of hardware devices and communication interfaces. A functionality which greatly assists novice programmers is the Embedded Component (known as 'beans' in legacy versions of CodeWarrior, such as 7.1.2.) Beans are modules which encapsulate a set of functionalities designed to accomplish the bean's objectives. One must simply indicate which type of peripheral to be used, enter settings, and then Processor Expert generates the code necessary to use the functions the user has indicated. In this project, bean wizards were used extensively to create code modules which manage hardware peripherals on the M52259Demokit.

\section{2 - CodeWarrior Programming}

This section explores the direct application of CodeWarrior Integrated Development Environment (IDE) to the Power System Simulator. When designing the programming scheme, several key functionalities were considered. The fundamental objective is to manage the digital switches which will reconfigure the routing of power on the simulator. Secondly, a Human-Machine Interface was developed to provide debugging and validation assistance when working with the MCU's. Finally, two optional functionalities will be examined, interface to the ADE7758 Polyphase Energy Meter, and Analog to Digital Converter for Current/Potential Transformers.

\subsection{1 - Electronic Switches and Status Indication}

Other than hosting the MGMS, the primary function of the M52259Demotkit is to handle the operation of the electronic switches installed on the Power System Simulator. The MCU is capable of taking input from a software agent a human operator. Because the development of agents is beyond the 
scope of this thesis, the manual operation of switches will be covered in this section. The programming used to handle agent interaction will be very similar to what is used to manage human operation.

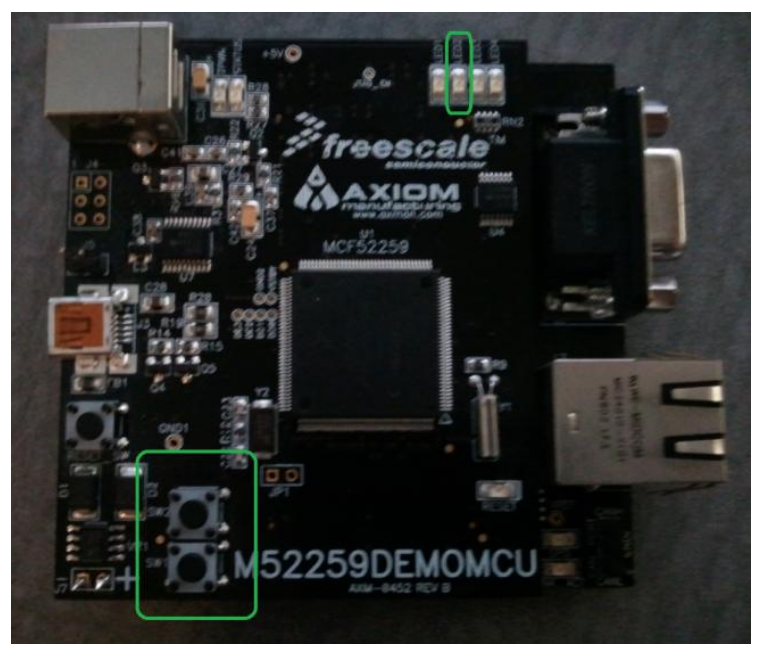

Figure 21: Switches and LEDs for Electronic Switch Control

A human operator will control the switching of the devices by use of two push-buttons on the front of the M52259Demokit (see figure above). In CodeWarrior, this is managed by enabling external interrupt for each of the pins connected to the pushbuttons. When the CPU detects a falling logic edge on either pin, a subroutine is triggered. When switch one (bottom switch) is pressed, the subroutine writes the value 'TRUE' to both the switch and indicator 'PutVal' functions. What this does is apply a high voltage to the pins associated to the switch and LED indicator. The LED indicator was assigned to ensure that the user knows the status of the switches by simply glancing at the modules. An identical but opposite subroutine occurs for switch two (top button). A total of four hardware beans and two subroutines manage the entire process, with the only direct input from the user or agent is to trigger the event. A sample of the code used to initial the switch can be seen below.

\section{Code Sample for Electronic Switches:}

void Sw1on_OnInterrupt(void)

$\{$ TriacStatus_PutVal(TRUE);

TRIAC_PutVal(TRUE); 
Processor Expert takes user input and names the methods in the program from what the user defines. All of the hardware manipulation is hidden under this manner. This makes rapid development of an implementation quite simple, once the user is familiar with the architecture of CodeWarrior and Processer Expert. A snapshot from the CodeWarrior IDE used to create this functionality can be seen

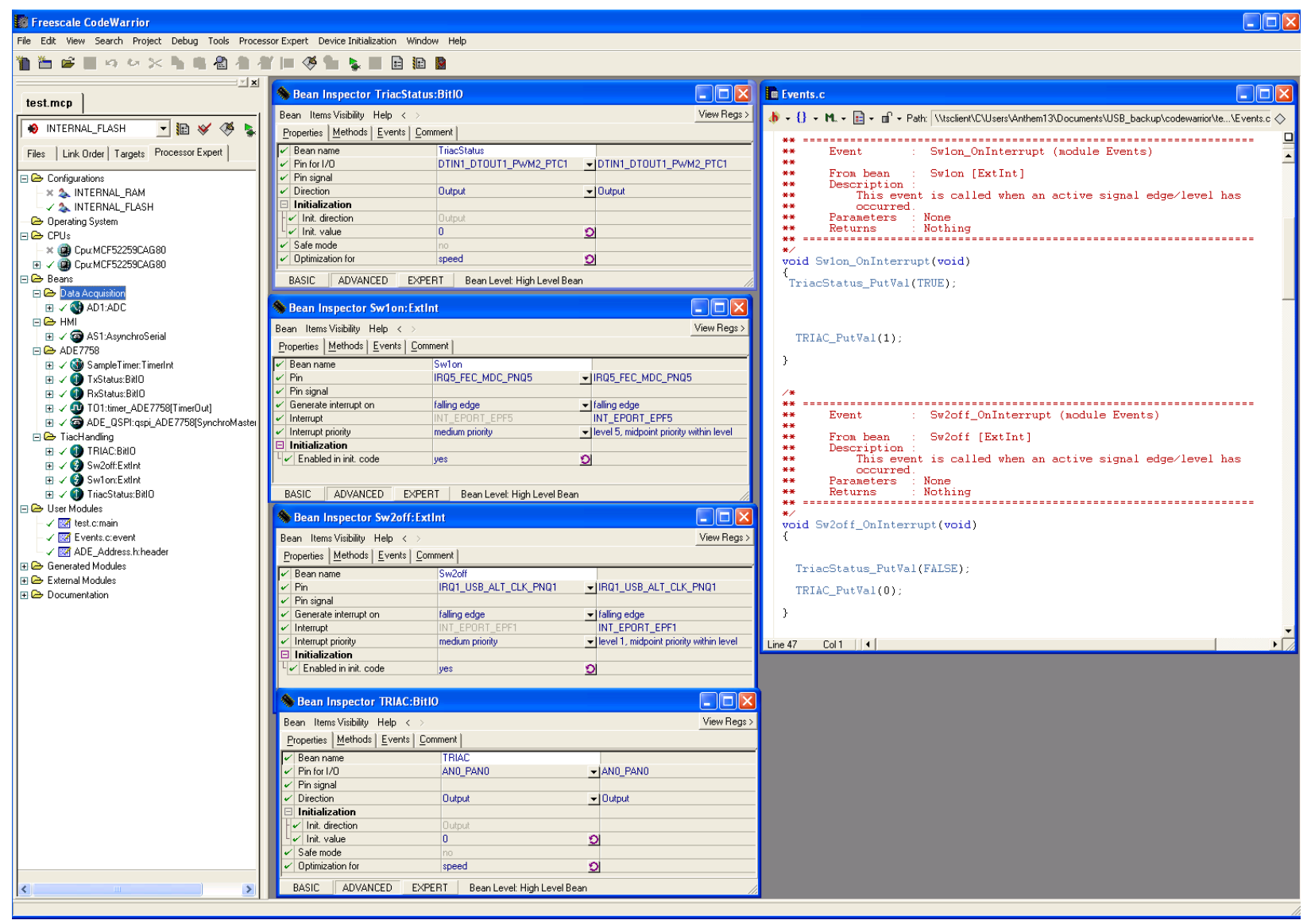

Figure 22: Development of Electronic Switch Control

\subsection{2 - Human Machine Interface}

Though the platform is intended to run without human interaction under normal operating condition, the M52259Demokits are programmed to interface with mobile workstations through RS-232$\mathrm{C}$ serial communication. This provides an easy method for data exchange and debugging.

This is done by attaching a serial cable from a PC or laptop to the M52259Demokit and initializing a serial communication program, such as Microsoft HyperTerminal. Settings have been chosen to reflect industry standards, as seen in the table below. 
Com Settings for HMI through RS 232

\begin{tabular}{|l|c|}
\hline \multicolumn{1}{|c|}{ Parameter } & Value \\
\hline Data Speed (Baud) & 9600 \\
\hline Data Bits & 8 \\
\hline Stop Bits & 1 \\
\hline Parity & NONE \\
\hline
\end{tabular}

At current state, the HMI has been used to validate communication with the ADE7758 Polyphase Energy Meter, but the purposes for its use are limitless. It is anticipated for a user to communicate directly with the MAS through this interface, gather information about status and agent operation during contingencies.

\subsection{3 - SPI Interface with ADE7758}

The Analog Devices ADE7758 Polyphase Multifunction Energy Meter is a three phase energy meter Integrated Circuit capable of processing a wide range of information, including active, reactive and apparent energy, frequency, RMS values for voltage and current, temperature, and waveform data. It incorporates second order $\Sigma$ - $\Delta$ (Sigma-Delta) ADCs, a digital integrator, reference circuitry, and internal

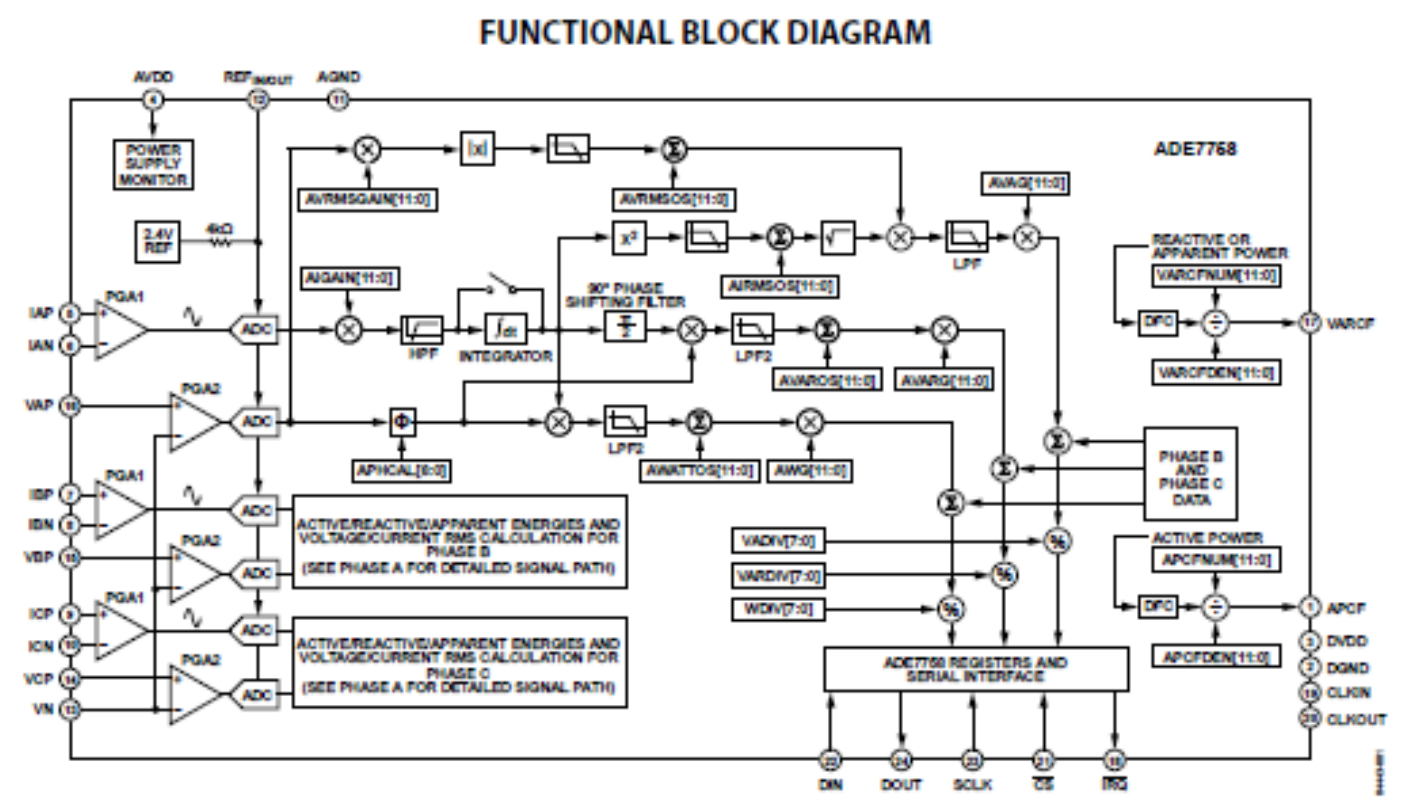

Figure 23: Block Diagram of ADE7758 
signal processing.

Not only can the chip provide all electrical information for an agent, but it also takes the burden of signal processing and computation from the host microcontroller. The MCU must simply request the information and the ADE7758 will respond. The transfer is done over the commonly implemented Serial Peripheral Interface (SPI).

The ADE7758 transfers data under two scenarios. The first is when the MCU polls the chip for information when it is needed. In this manner, the MCU can request any type of data at any time. The second scenario is an interrupt-driven transfer. This occurs when a threshold limit within the ADE7758 is violated. The chip then sends signal to the MCU, which responds by retrieving information from the sensor chip.

In either communication scenario, the MCU first writes to the internal communications register of the ADE7758. The data written to this register indicates the register of interest and if the MCU will read or write to the register. The chip then either receives information to write to the register of interest or delivers the contents of the register to the MCU. In the polling mode, the MCU simply sends a 'write' command (say to, for instance, the Phase A RMS Current register) to the chip, and the chip will respond with the contents of the register. For an interrupt driven exchange, the ADE7758 will indicate an interrupt event by driving the IRQ line low. The MCU recognizes the event, and issues a 'read' command with the address of the IRQ Status Register. The ADE7758 then replies with the contents of the Status Register, which indicates which threshold has been violated. Examples of such cases are voltage limit violations, or

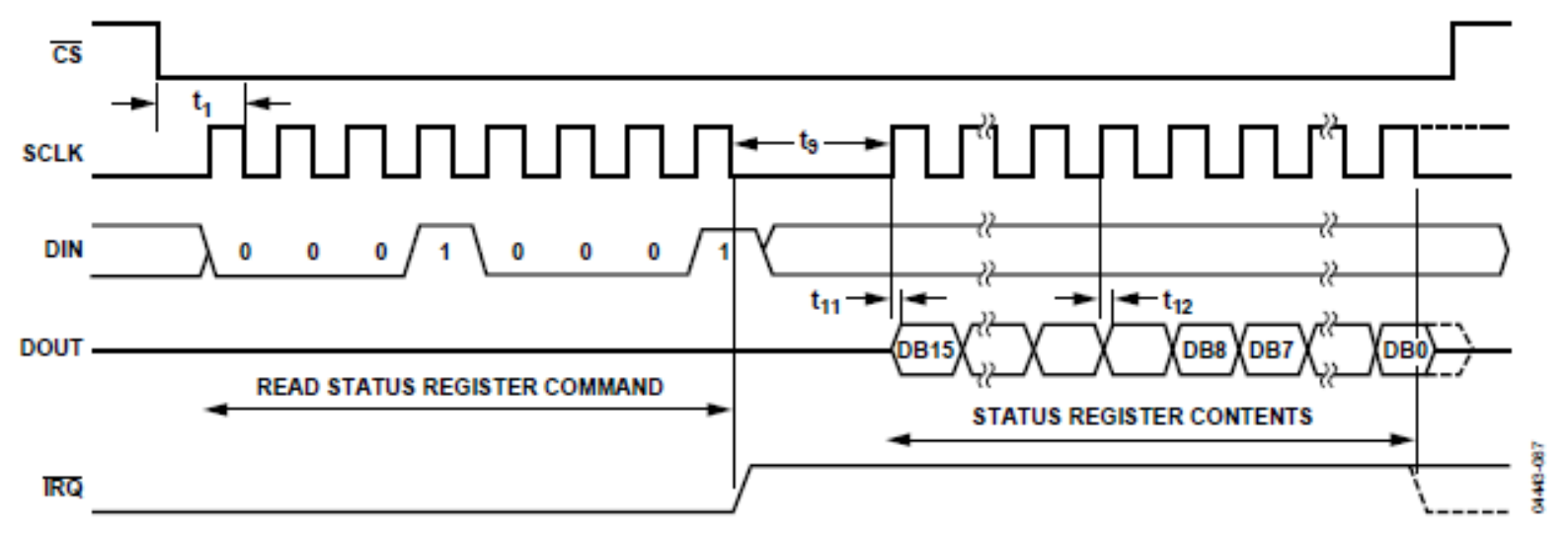

Figure 24: Interrupt Event Sequence 
the filling of the waveform data register. This is convenient, because this functionality can remove a large amount of processing requirements from the MCU.

Despite the benefits discussed, a major problem concerning its implementation is the fact that it cannot be exported to field distribution systems. A major facet of the MGMS Hardware Platform is its analogous nature to industry distribution systems. Using a data acquisition device that is not deployable in the industry would jeopardize the practicality of the system. Hence, though the ADE7758 can very well be used, the SEL relays will be the primary data acquisition devices used on the power simulator.

\subsection{4 - Analog to Digital Converter}

The final functionality incorporated into the M52259Demokit is the Analog to Digital Converter. Though the MGMS is designed to retrieve operating condition data from the SEL relays, it is possible that an agent will be desired in a location that is not also host to a relay. In this condition, it would be necessary to obtain some sort of information about the electrical quantities in that area. To overcome this obstacle, it would be easy to implement a Current Transformer or Potential Transformer to allow the agent access to information in its vicinity.

The M52259Demokit incorporates an eight channel 12-bit ADC with as low as $1.7 \mu$ s conversion time. Reference voltage can be internal or external. The specifications are more than suitable for modern power system applications. Provided the situation presents itself, the package is well prepared to be implemented in a range of environments. 


\section{Chapter Six - Hardware Integration Results}

All of the aforementioned aspects constitute the vision of the upgraded Power System Simulator. This chapter explores the integration of the protection, MGMS hosting, and reconfiguration hardware into the PSS.

\section{1 - Integration of SEL Devices to the Power System Simulator}

The deployment of SEL devices to the PSS can be divided into two efforts: the placement of the relays and the connection of the data systems. The PSS had to be physically modified to accept the relays and computing system described in Chapter 2. The data connections constitute the wiring of existing CTs and PTs to the digital relays, as well as the RS-232 and HMI hardware.

\subsection{1 - Relay Placement}

The relays donated by SEL were selected to parallel the existing protection system came with the PSS. Michael Spencer determined the appropriate locations for the devices and installed them accordingly. The diagram below shows the placement of digital relays.

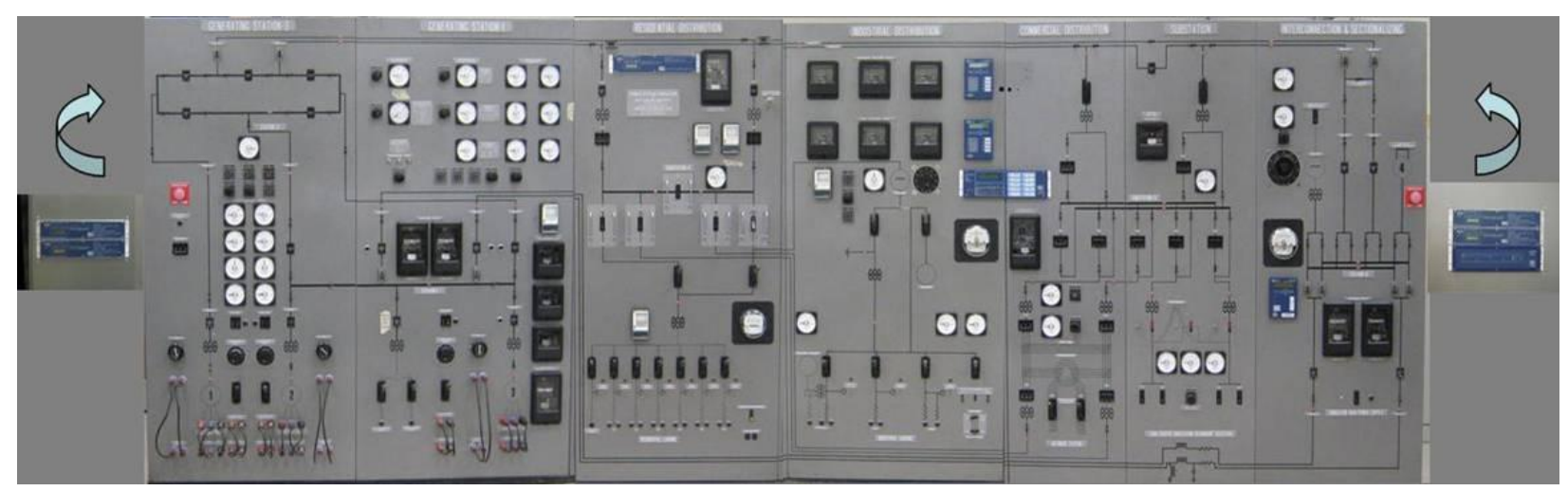

Figure 25: SEL Relays Integrated to the Power System Simulator (Spencer, 2010)

On the right-most panel are two SEL-351 Directional Overcurrent Relays and the SEL-3351 System Computing Platform. The 351s take responsibilities of the two electromechanical directional relays which monitor the incoming feed from the utility. The next relay to the left is the SEL-734 Revenue Meter, which is paralleled with the antiquated $\mathrm{kWHr}$ dial. The next cluster is composed of two SEL-751A Feeder Protection Relays and the SEL-351S. The 751As monitor the feeders which run along the top of the PSS, while the 351S is paralleled with the reverse power relay which protects the substation region. The next placement is the SEL-387A which parallels the transformer differential relay in the residential loading section. Finally, two SEL-351s are placed for directional overcurrent in the Distributed 
Generation section of the PSS. High resolution photographs of these placements can be seen below, as seen on the simulator from right to left.

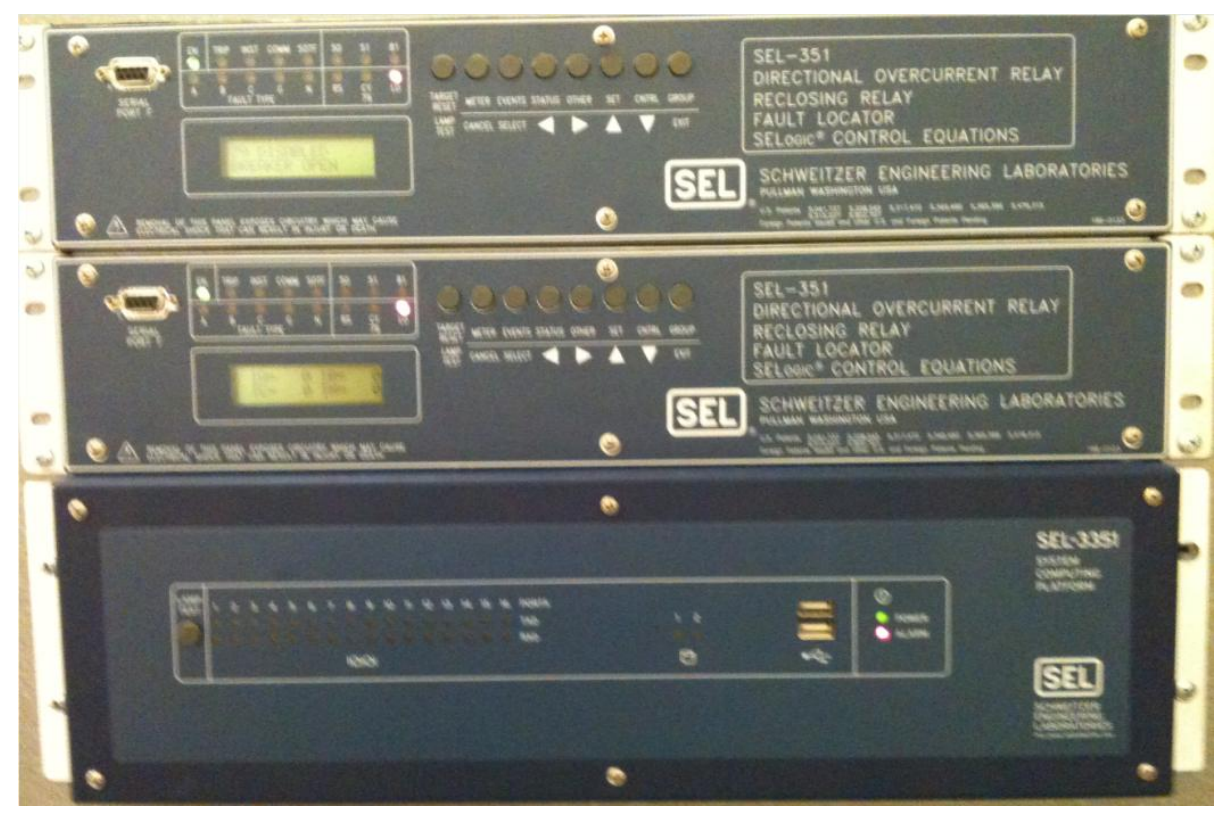

Figure 26: Two SEL-351s and SEL-3351

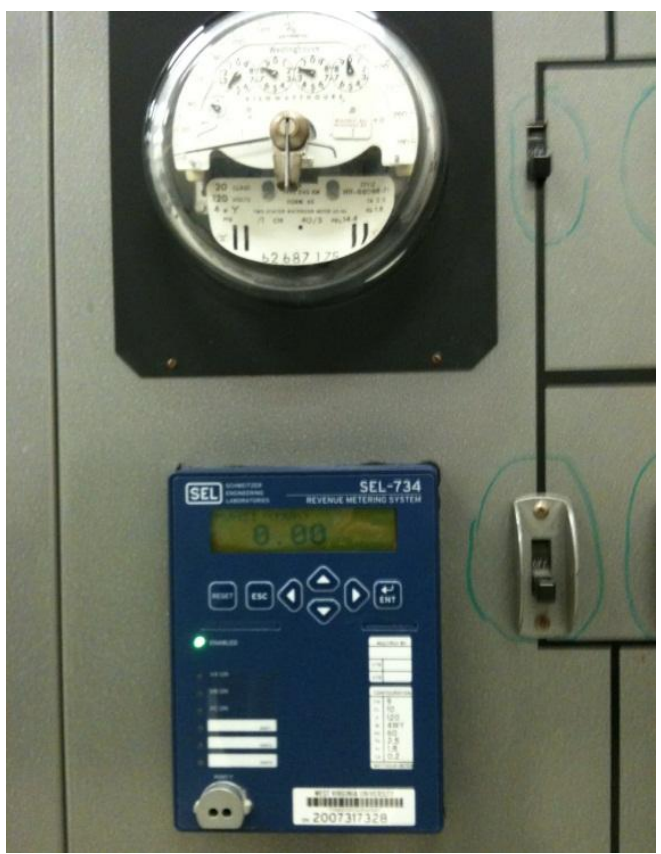

Figure 27: SEL-734 


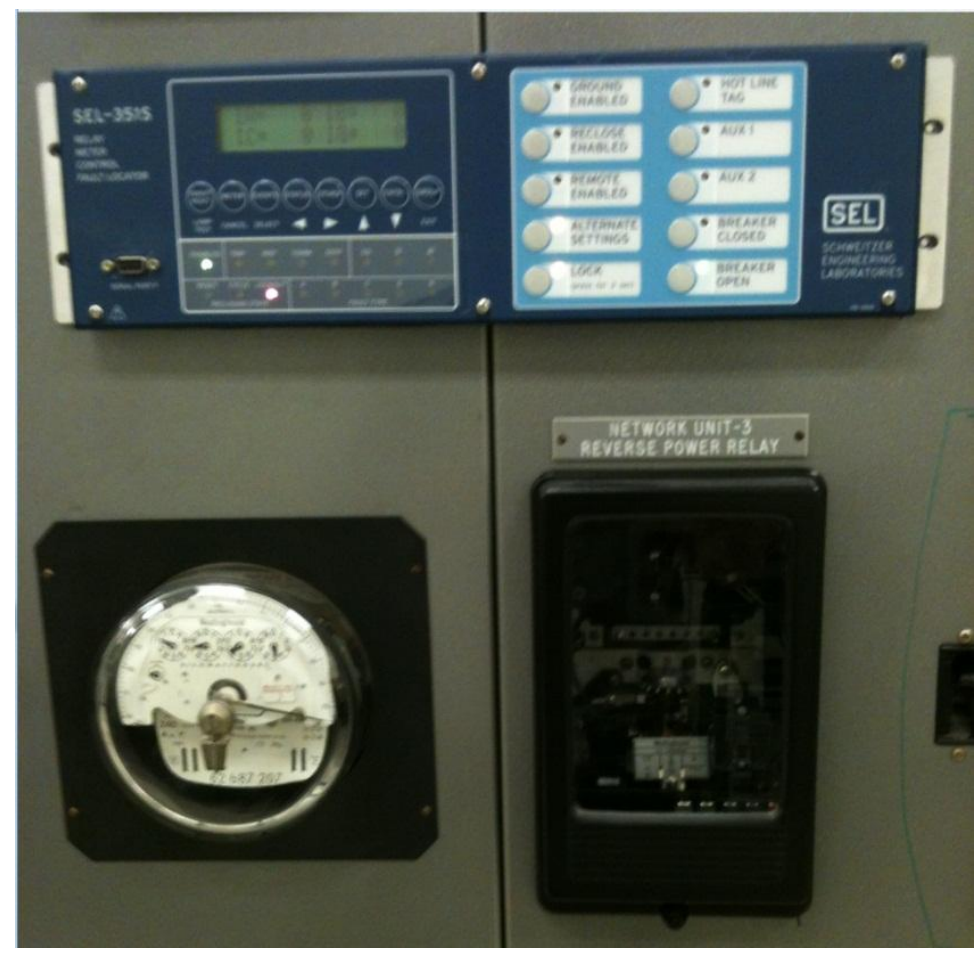

Figure 28: SEL-351S

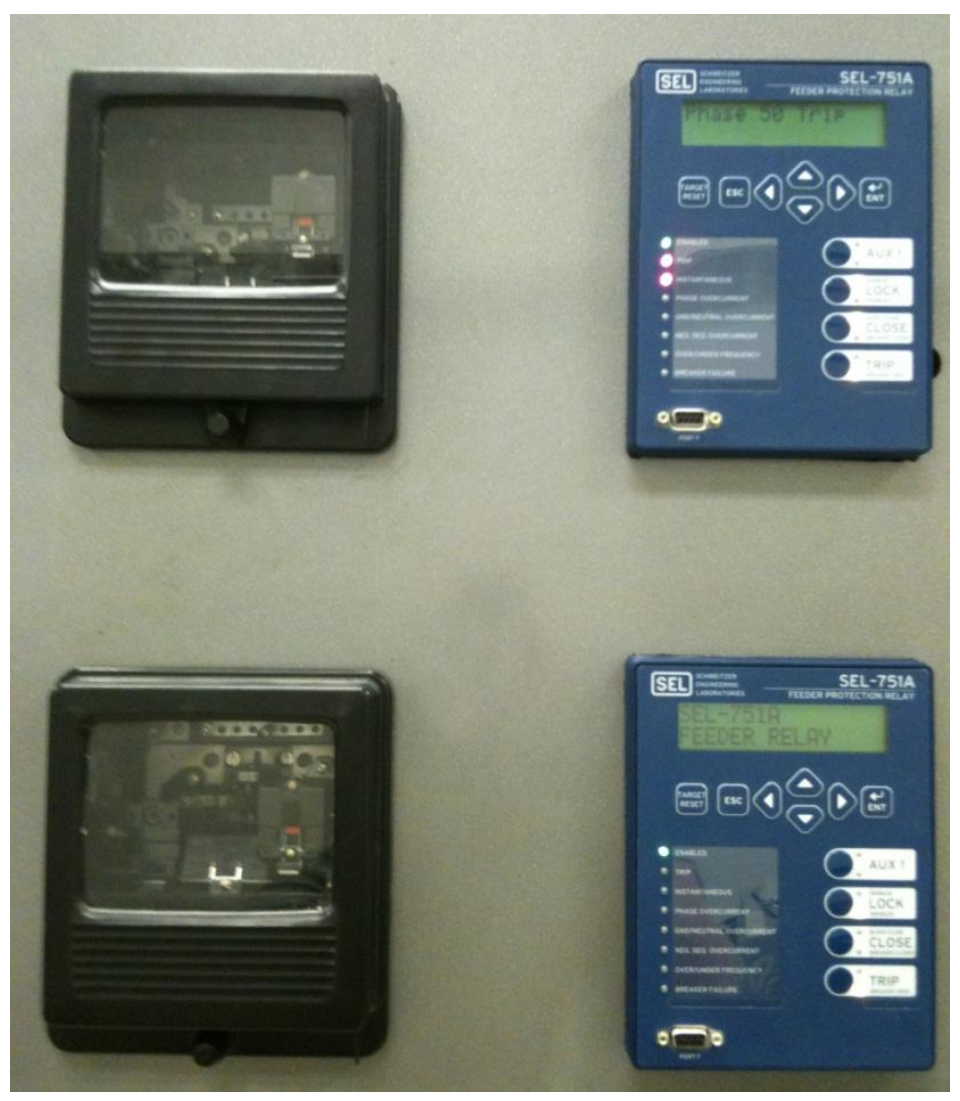

Figure 29: SEL-751A 


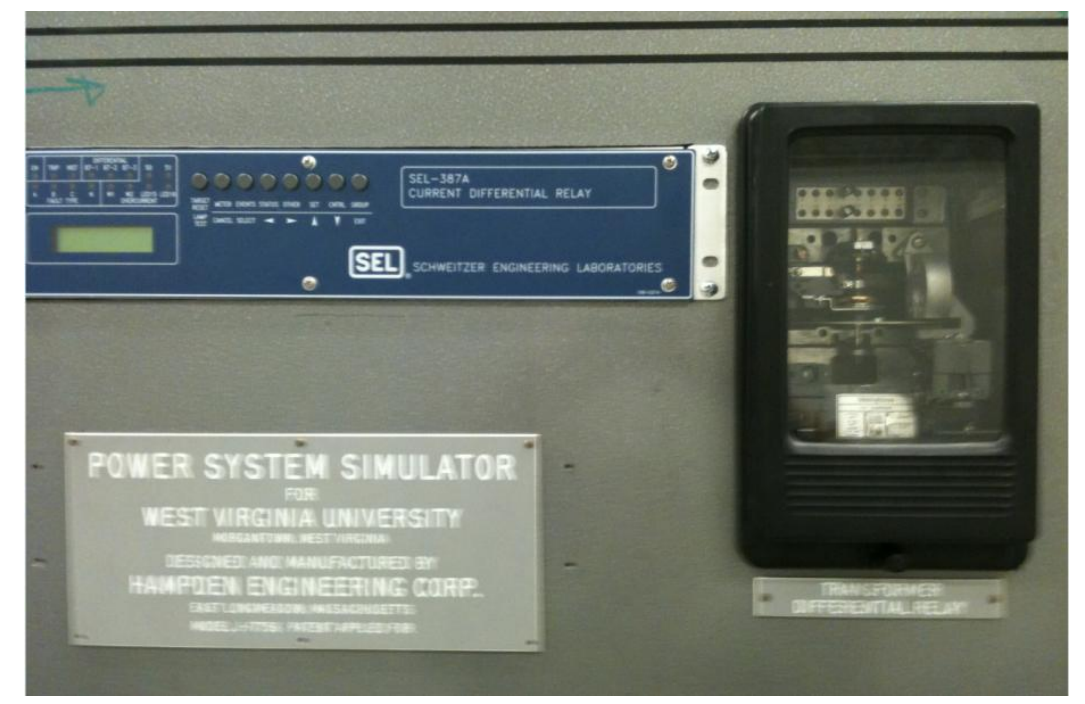

Figure 30: SEL-387A

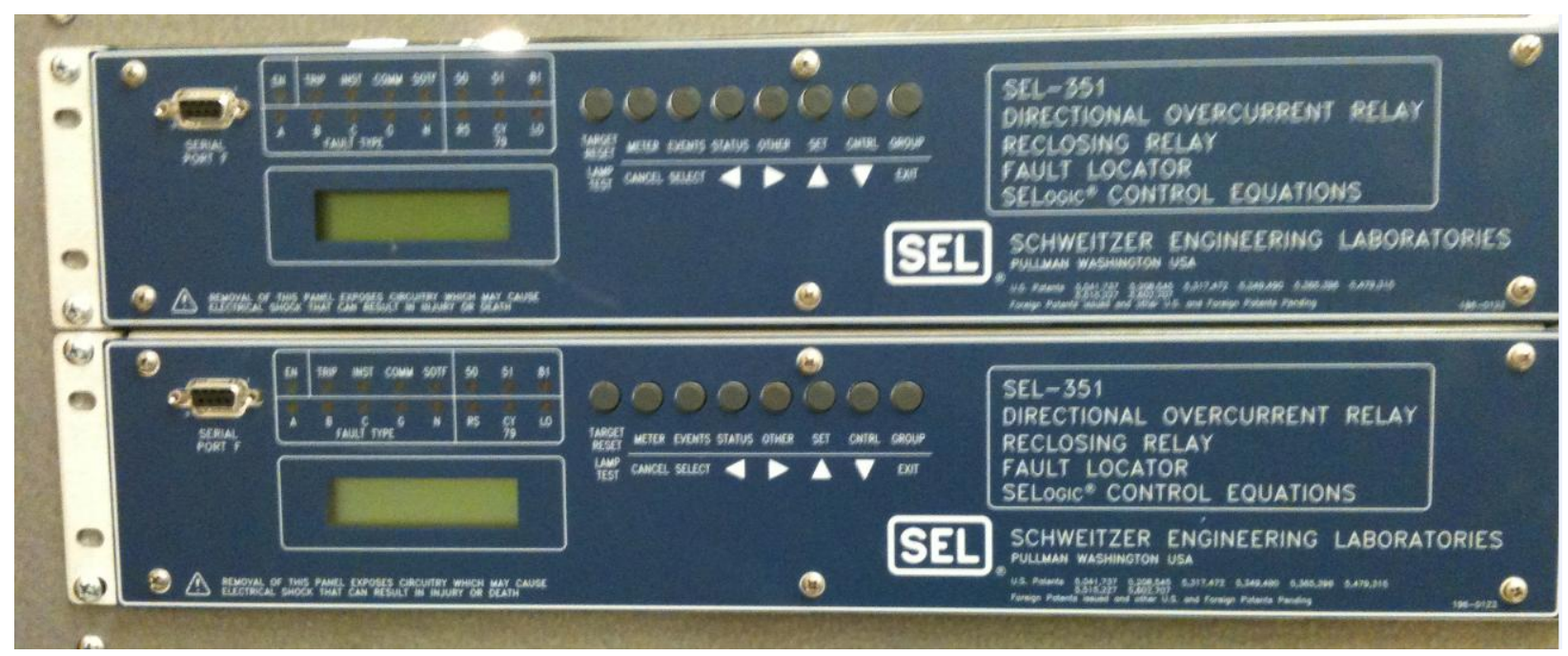

Figure 31: Two SEL-351s on DG Interface 


\subsection{2 - Data Connections}

As discussed in Chapter 2, The SEL protection network supports many communications protocols. For the purpose of the Power System Simulator, RS-232 was chosen for its ease of use and inexpensiveness. The maximum required distance for communication for cabling is about thirty feet, allowing SEL-provided cables and extensions. The SEL-5531 has a built-in 16 port RS-232 array, so no additional hardware is required to interface the entirety of the protection network.

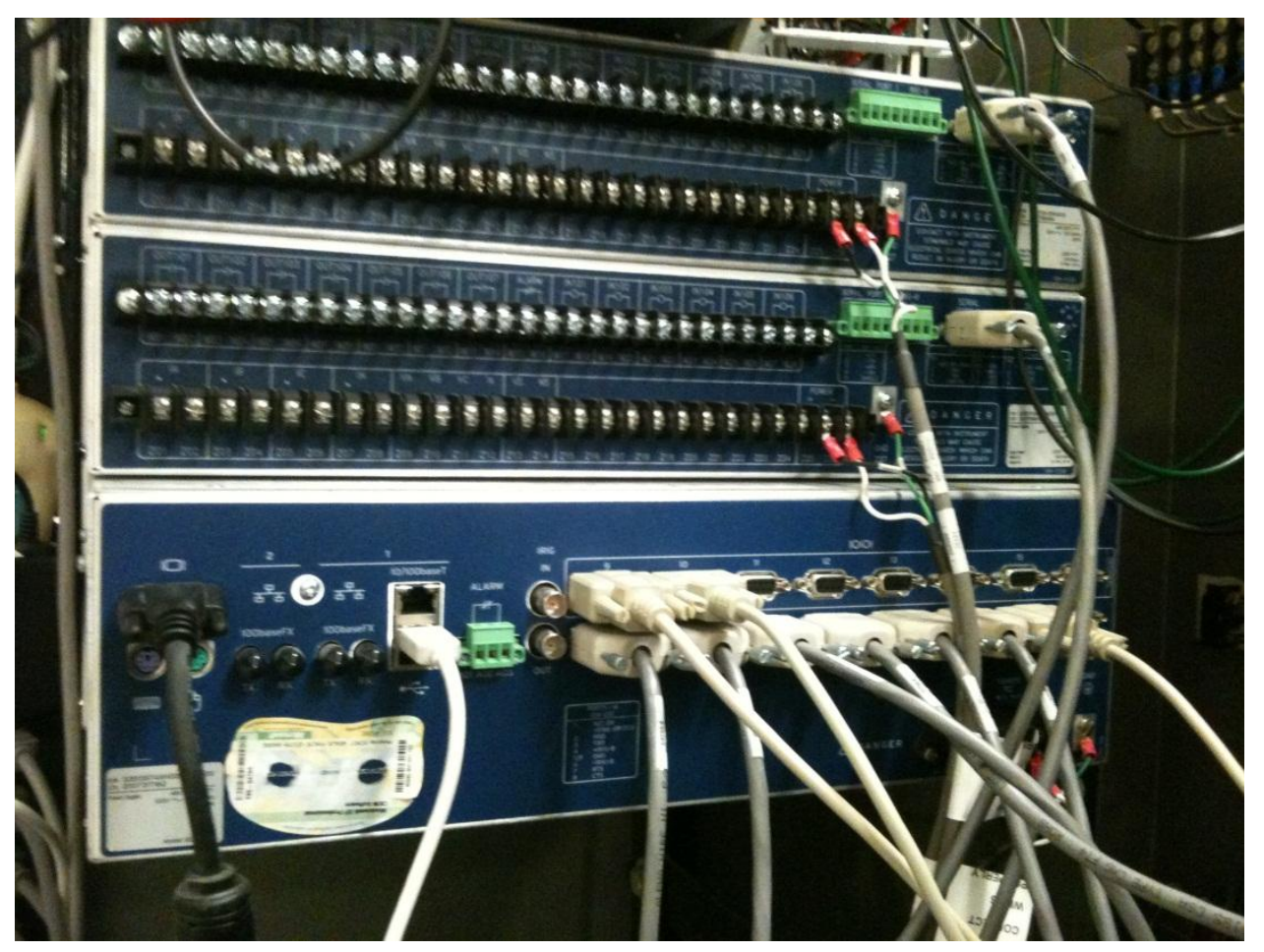

Figure 32: RS-232 Connections on Back Panel of SEL-3351

The 700 series relays' default settings allow for the devices to interface with the 5531 without any additional setup. However, they 300 series relays require having the Baud rate changed to 9600 for the system to work together seamlessly. This is done by means of the front panel of the relays. A user may enter the data settings after entering passwords which allow administrator rights. Once this is done, the user can enter the settings through the Quickset program to change settings further. 


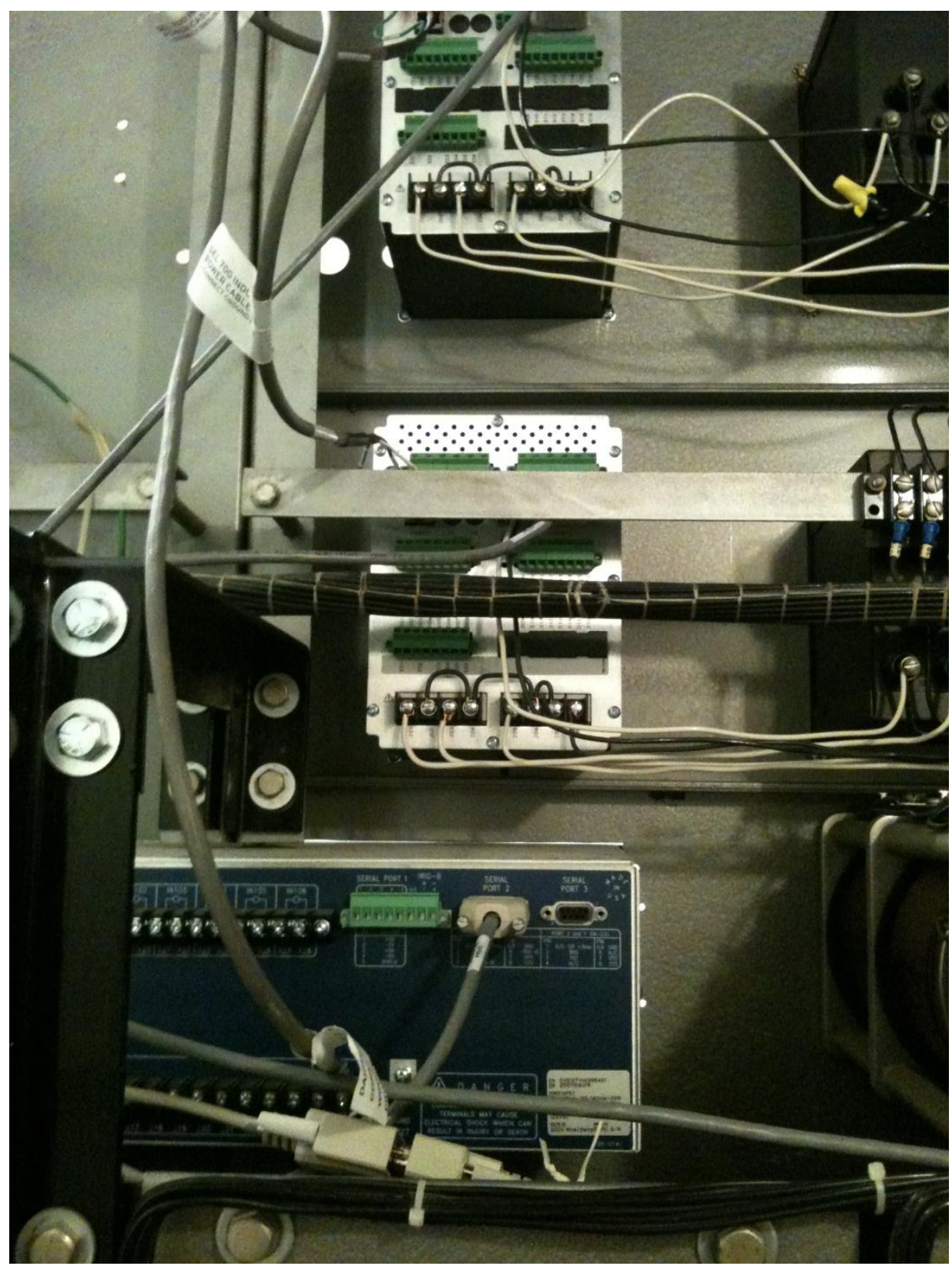


Figure 33: SEL-751 and 387A with Data Connections

\subsection{3 - SEL 5030 AcSELerator QuickSet}

Although the hardware capabilities of the protection system are paramount, it would be valueless without software to link the devices and convey information to the system operator. This is why the SEL 5030 AcSELerator QuickSet software package was developed. Functionality includes management of relay settings, a waveform view, development tools for SELogic control equations, graphical logic, an integrated HMI, and more. 
The QuickSet software was installed on the SEL-3351 which is located on the right side of the power system simulator. QuickSet is then configured to communicate with the relays through the RS-232 communication ports on the rear of the 3351. Once the devices establish the communication channel, the user is able to access the full array of relay settings. Communication setup, time curve settings, as well as sampling rate and other parameters can be manipulated. Also, the user can initiate the Human-Machine Interface, which shows real-time data sampling including phasors and waveform data.

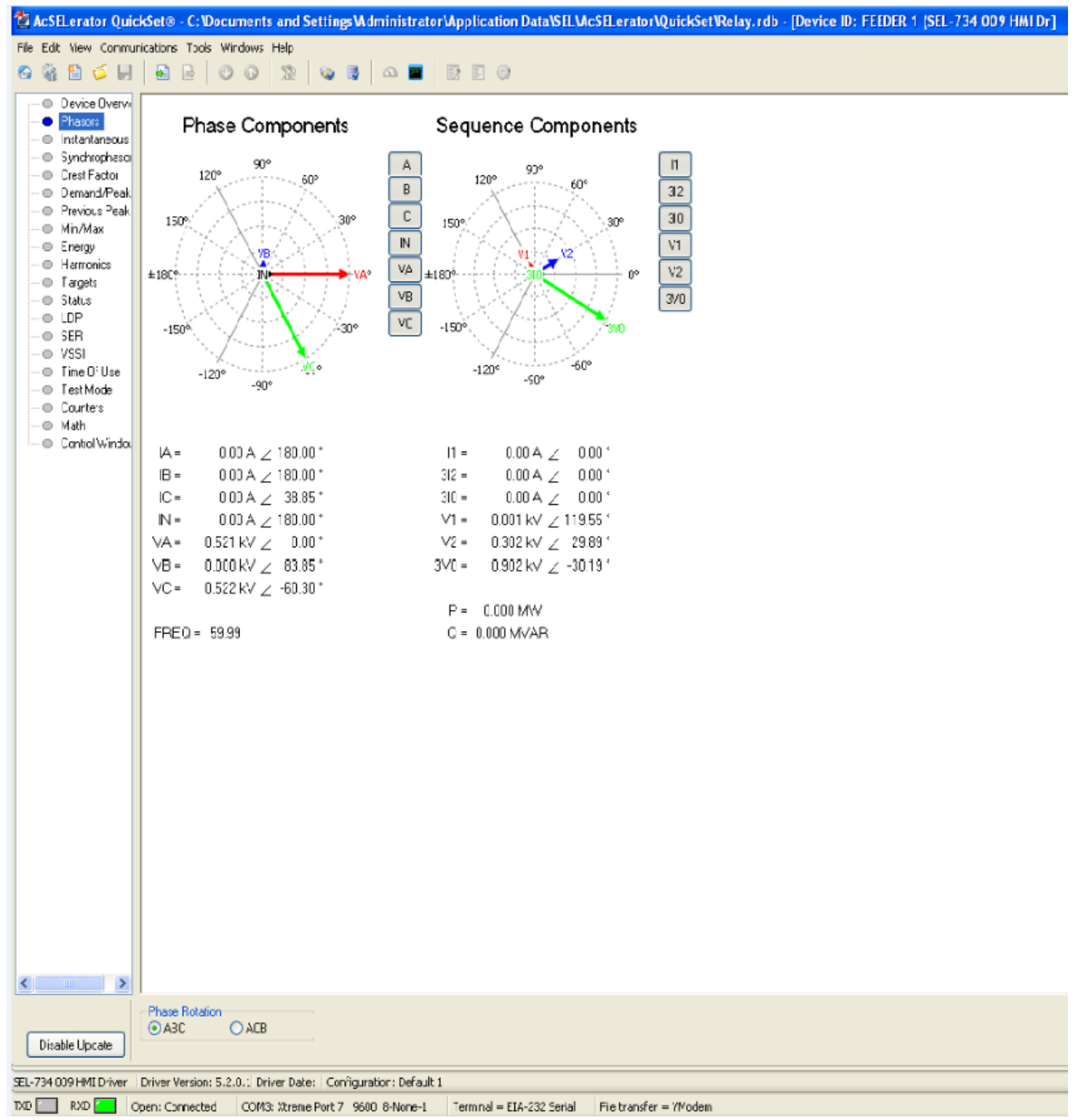

Figure 34: Screenshot of SEL HMI 


\section{2 - Electronic Switch Validation}

A fundamental aspect of the hardware platform for a MGMS is the ability to intelligently reconfigure the topology of the system. This requires devices capable of performing switching functions and devices which can host the algorithms as well as interface the switching devices. As formerly discussed, the M52259Demokit is very flexible and is fully capable of hosting agents as well as interfacing switching devices. Yet, finding devices to perform the switching functions on the Power System Simulator was a challenge, due to the uncommon nominal voltage level of the machine. Despite this obstacle, an electronic relay made by Omega was identified to be a solution to this problem.

The Omega G3NA-420B-DC5-24 solid state relay is specified as capable of handling loads from 180 to $528 \mathrm{VAC}$ at 0.2 to $20 \mathrm{~A}$. This is nearly ideal for the situation at hand, as the PSS is often ran at loads lower than 600 volts and rarely experiences current over 3 amperes. This provides sufficient overhead for transients and current interruption capabilities. The relay is a voltage driven device with specified minimum turn on at 3.75 volts. Integration is simple with only four inputs: logic for triggering, and the load itself. This yields a high level of flexibility when considering interface with logical systems.

\subsection{1 - Preliminary Testing}

Preliminary tests were performed and the device was accepted as the choice for paralleling many of the mechanical switches found on the PSS. Tests indicate that the minimum turn on voltage for the devices we acquired are at 2.6 volts, well under the 3.3 volts which are output by the M52259Demokit. Furthermore, current interruption was proven at 5 amps at $208 \mathrm{~V}$ as well as 3 amps at 550 volts. This is suitable for not only the main lines in the Power System Simulator, but also the Distributed Generation stations on the left side of the simulator. 


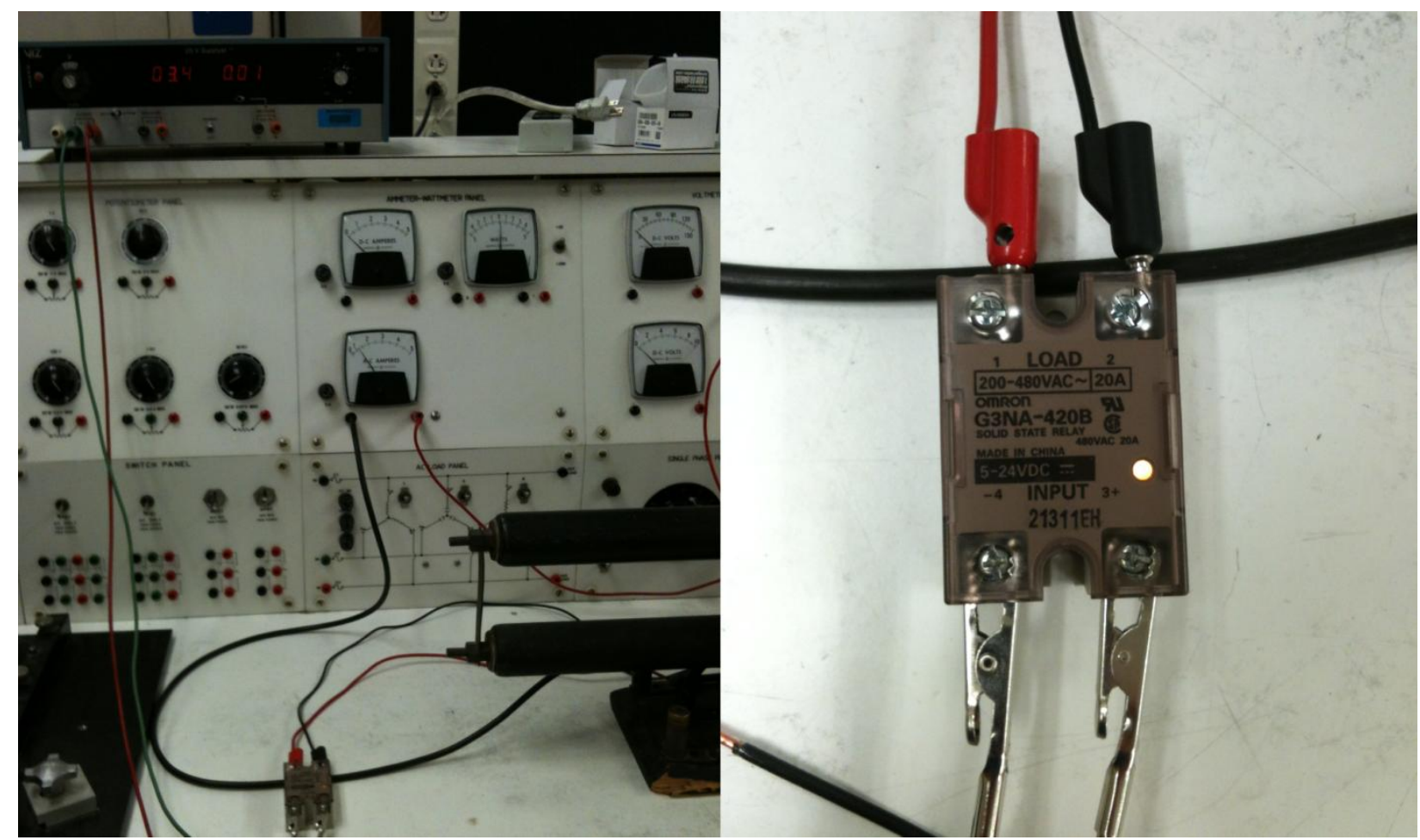

Figure 35: Test Setup of G3NA-420B-DC5-24

Once the preliminary tests were performed, a switch location was chosen which will aid in a demonstration lab showcasing a fault location algorithm. Two of the devices were installed to parallel the switch (single phase line to line load). The relays were placed visibly directly behind the mechanical switch, mounted near two capacitors and the transformer fault selector. This will not only offer a clear visual check for a user, but the metal panel will also aid in heat dissipation.

The devices were tested as they would be operating during the algorithm. The M52259Demokit performed the triggering of the devices while the PSS was operating at 550 volts. The load being switched was approximately 2 amperes. Seen in the figure below, the status indicator LEDs can be seen on both the relays and the microcontroller. When the MCU was triggered to send the command, the switches immediately offered a conductive path for power to reach the loads.

The test was a success, verifying the choice of the relay to be implemented upon many of the switch locations on the simulator. Key reconfiguration points will be identified, and the relays will be exported to the PSS for use in MGMS development and testing. 


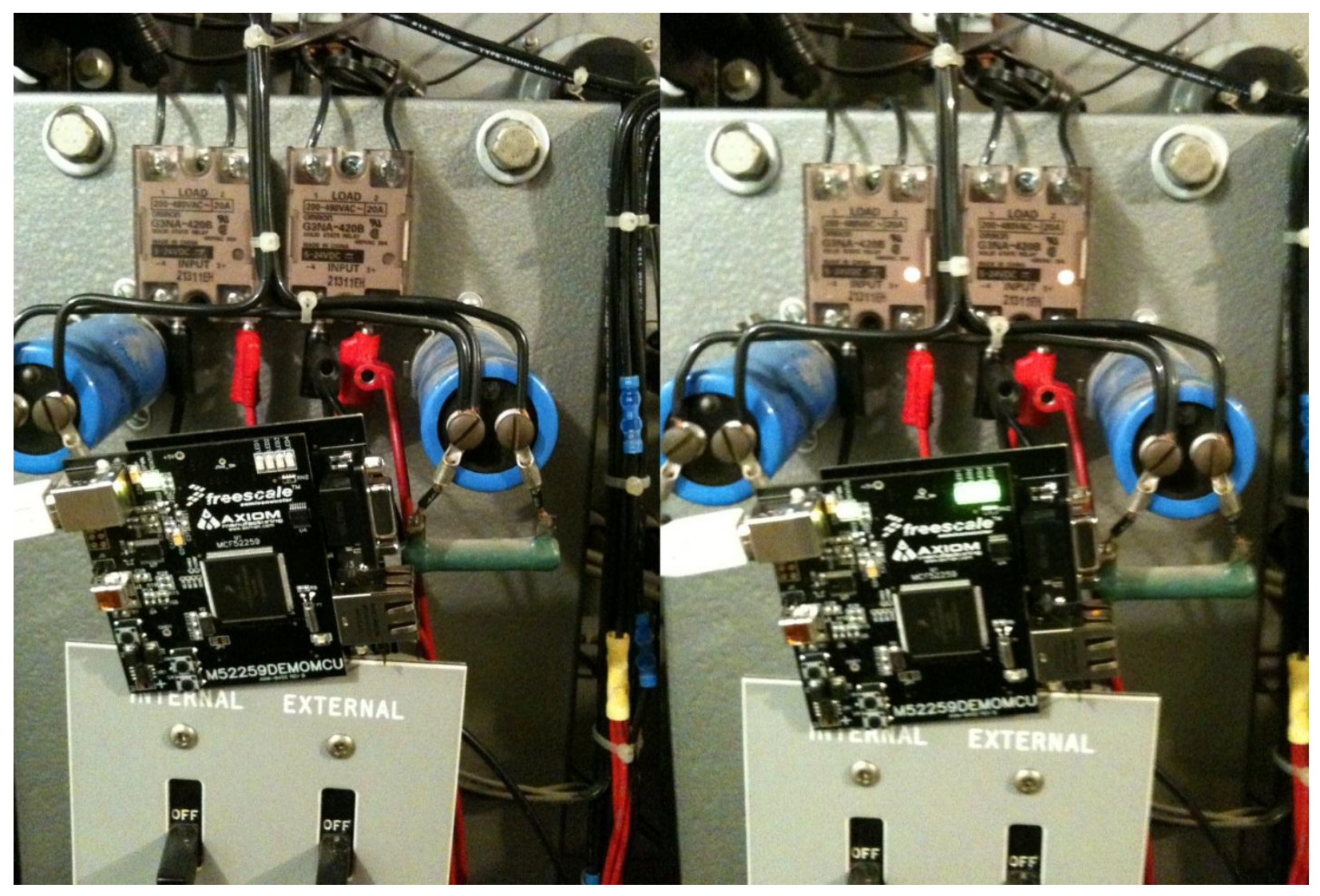

Figure 36: Solid State Relay Deployed to the Power System Simulator

\subsection{2 - Waveform Analysis and Comparison}

A waveform analysis was run to compare the performance of the Omega relays to the discrete TRIAC devices which have been used for previous endeavors on the PSS. The objective is to verify that the relays operate as intended and that there are no issues which may cause the devices to malfunction.

The test setup used to compare and contrast the G3NA-420B-DC5-24 and the NXP BTA208X was identical to the preliminary testing except for the introduction of the Yokogawa PZ4000 Waveform Analyzer and analog meters. The devices are connected in serial with a large variable resistor. The resistor was set to $\sim 125 \Omega$ so that the devices would experience currents similar to what would be a worst case scenario within the Power System Simulator.

The Yokogawa PZ4000 Waveform Analyzer is a versatile device which allows a user to specify a wide range of parameters. Current, voltage, active and reactive power, impedance and angle, and frequencies of voltage and current were selected as parameters of interest for this procedure. The current 
sensing shunts were placed in series with the device and resistor, while the voltage leads were connected directly across the switching device. With the device connected and in 'off' mode, the voltage is applied to the circuit. The control signal is then applied to the device, and the waveform is recorded on the Yokogawa Waveform Analyzer.

The devices were tested under the harshest conditions that would be seen in the power system simulator. If chosen to be placed at the low voltage side of incoming distributed generation, the relays would experience up to 6 amps at 208V. The conditions were generated and the waveforms were captured under these conditions. The waveform of the Omega relay was also captured while functioning in the simulator to validate similar performance characteristics. The results from the Omega relay and the TRIAC are shown below, respectively.

The significant difference between the two waveforms can be seen at the zero crossing point of the current waveform. At this time, the circuit with the Omega relay experiences a voltage spike. By examining the axis of the graph, we can see that the spike is approximately 19 volts @ 208 volts load and 25 volts at 500 volts load. Though the package includes a snubber, an Omega representative indicated that sometimes the snubber is not sufficient and additional countermeasures may be employed. However, the voltage spike as it is poses no potential harm as it is far below the blocking capabilities of the relay.

One major benefit of the Omega relay over the TRIAC switch noted in the tests was the ability to dissipate heat energy. The G3NA is able to quickly remove heat from the switching devices by means of the built-in heat sink. However, the TRIACs must be fitted with an aftermarket device to properly deal with the losses. At the end of the experiment, the G3NA was still cool to the touch, while the NXP BTA208X was heated to the point that it was unable to be handled.

In conclusion, the G3NA-420B-DC5-24 is a convenient and effective module for use in the Power System Simulator. It is capable of handling all of the voltage and current levels with plenty of safety overhead. The solid state relay can be controlled by any common DC voltage used for logic. The packaging and safety considerations allow for rapid deployment with fairly little effort. Overall, the G3NA is an effective, convenient, and reliable choice for upgrading the Power System Simulator. 


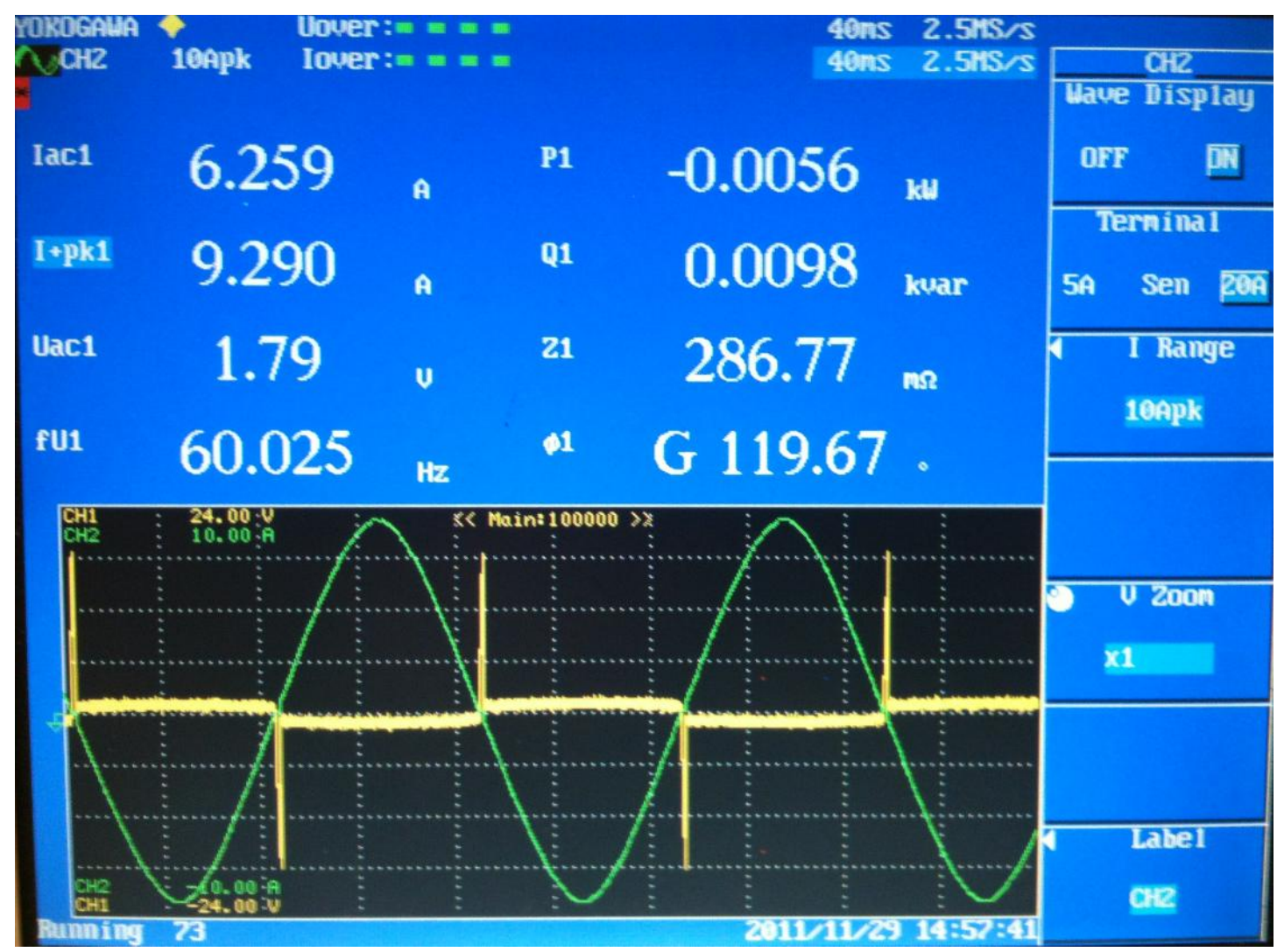

Figure 37: Omega Relay @ 208 Volts

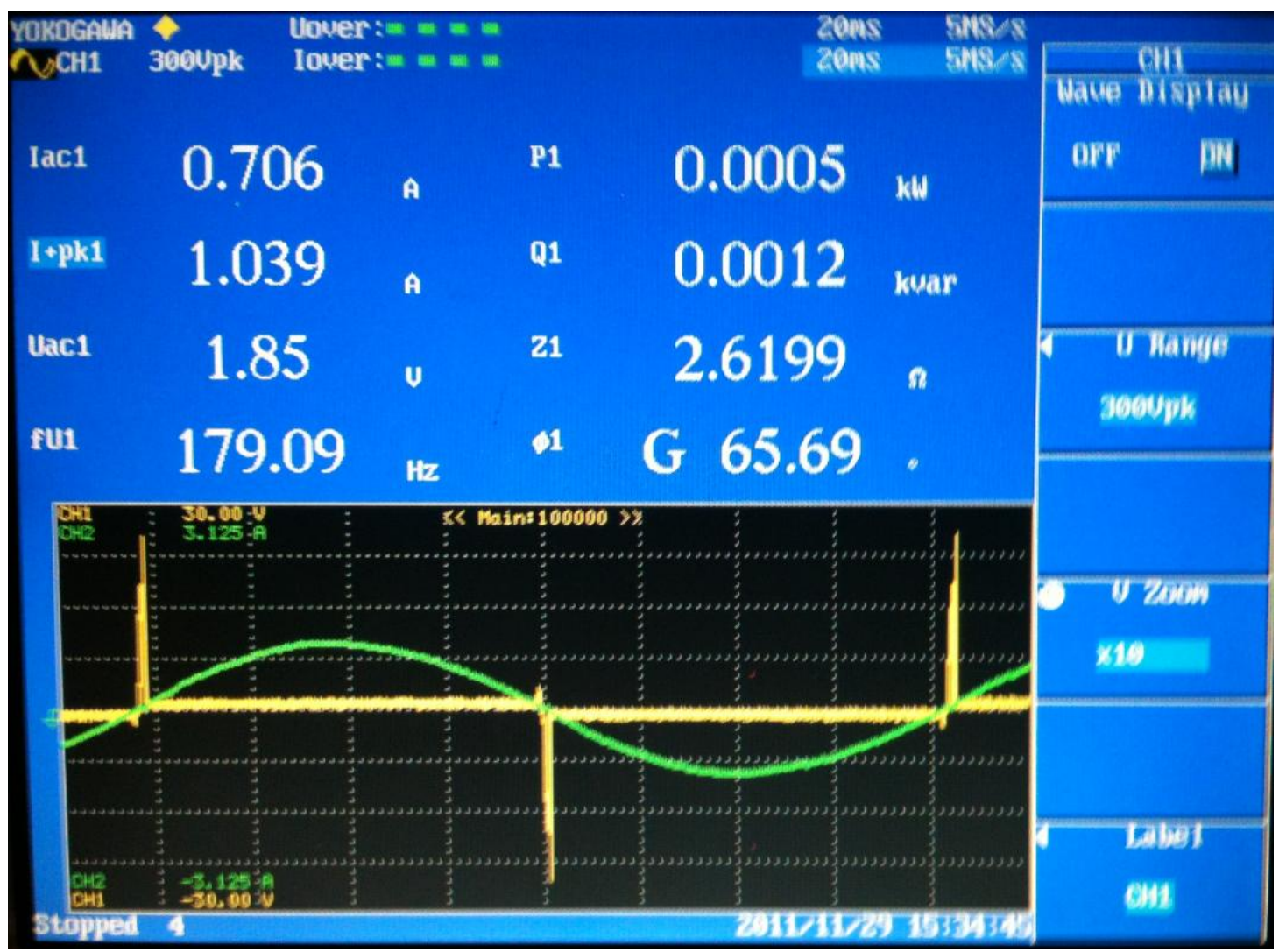

Figure 38: Omega Relay @ 500 Volts 


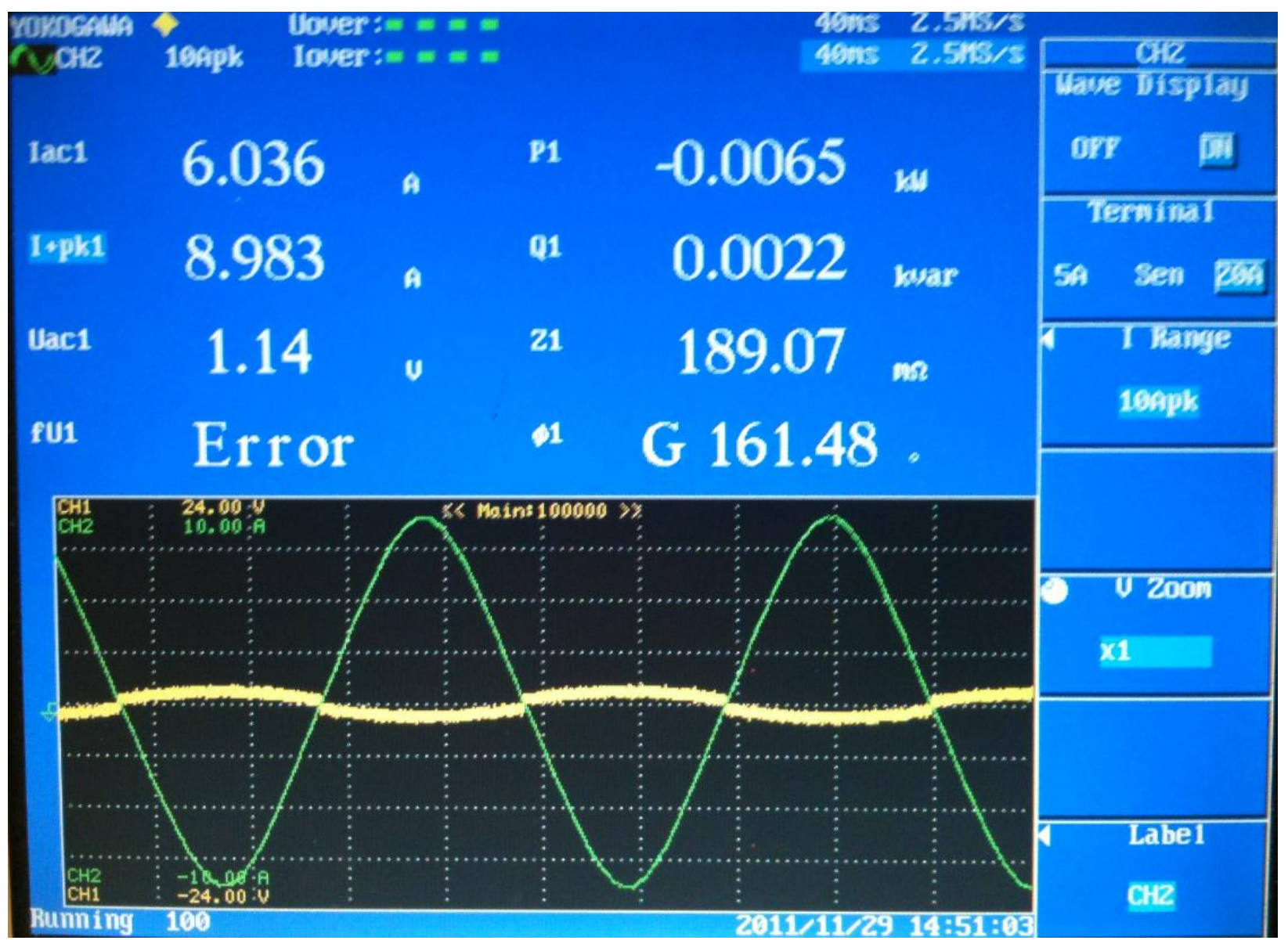

Figure 39: NXP TRIAC @ 208 Volts 


\section{Chapter Seven - Smart Grid Application}

\section{1 - Scenario Description}

A case study was developed to showcase the enhanced functionality provided by the hardware platform which has been described in the previous chapters. The study applies a Fault Location/Isolation/Restoration algorithm to the upgraded Power System Simulator by the use of distributed intelligence (agents) and electronic switches.

The PSS is configured to supply power to the Residential Loading area. A fault is simulated and the Multi-Agent System identifies the fault and isolates the faulted area. The MAS then identifies an alternative route for power and restores service to the load.

\section{2 - Algorithm Structure}

The MAS consists of five agent locations. For convenience, the agents will be referred to by their position going from right to left. Two of these, Agents 1 and 2, are at the source of the main feeders supplying power across the simulator. Agent 3 is immediately before the Residential Loading panel, while Agent 5 is at the end of the top feeder on the Generation panel. Finally, Agent 4 is positioned directly above the actual loads in the Residential section, and the interconnection to the bottom feeder. The locations of the agents are identified in Figure 40 by the green circles. The first four agents (all except residential) comprised Dr. Rabie Belkacemi's demonstration of a Multi-Agent Grid Management System. The fifth agent has been placed with the aforementioned technology, adding the ability for full reconfiguration and load restoration.

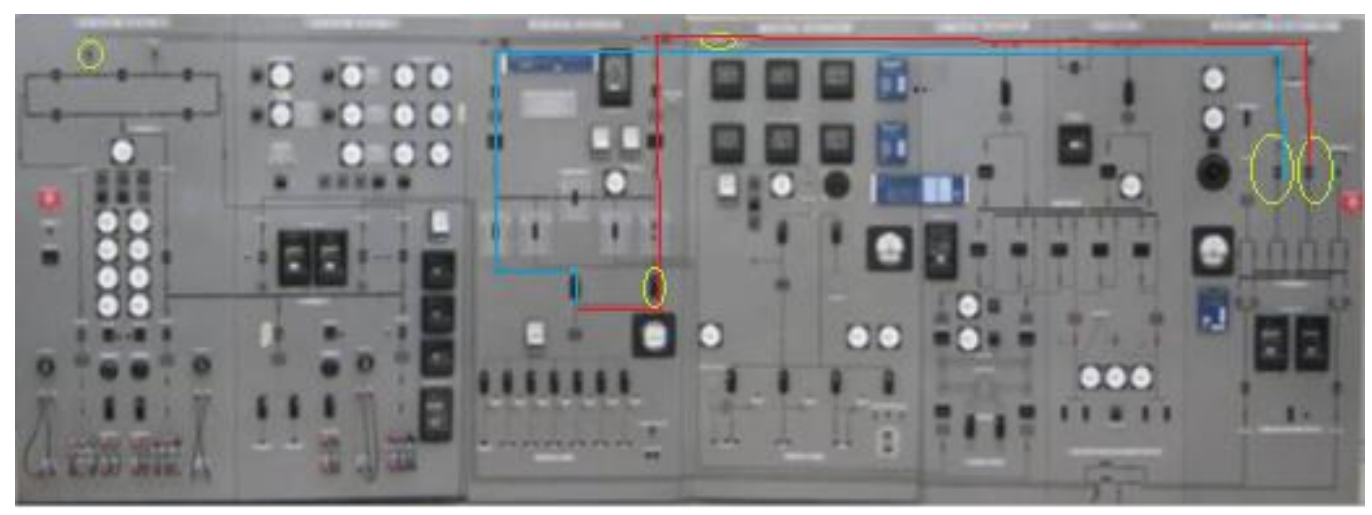

Figure 40: Case Study Scenario 
Each agent location has two important functions: interacting with the power system and interacting with the Multi-Agent System. The agents observe their environment using current transformers and Analog to Digital Converters, and interact with one another via wireless communication. By maintaining communication in regular intervals, the agents are able to protect the system from contingencies. A diagram showing such a FLIR communication interchange can be seen in Figure 41.

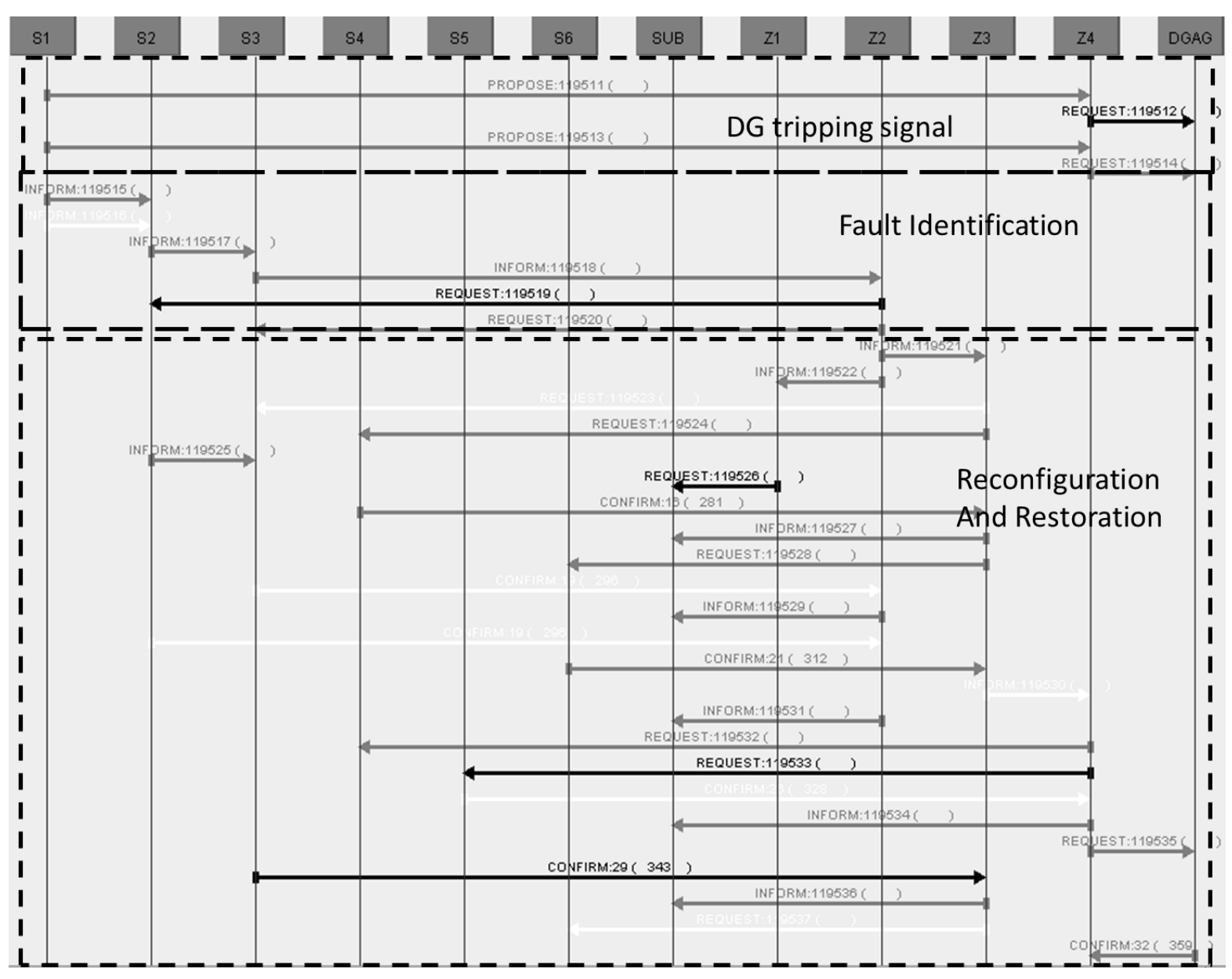

Figure 41: Example of Agent Communication 


\section{3 - Results}

The algorithm scenario is as follows: power is routed through the top feeder to the residential loads (see the red line in Figure 40). In this configuration, the agents' switches along the red line (1, 3, and 4) are normally closed, while the agent's switches along the blue line (Agent 2) are normally open. A fault is simulated by applying a large load based in the Industrial panel of the simulator.

The load draws a large current, which is detected by the agents along the top feeder. Agent 5, in the Generating station, does not see the fault, while Agent 3 does experience the overcurrent. Similarly, the Agent 4 does not sense the overcurrent, as the fault occurs in between Agents 3 and 4. The agents deduce that the fault must lie between them.

This causes the Agents 3 and 4 to open their switches, isolating the faulted area from the rest on the system. With the system stabilized, the MGMS attempts to maximize the service area. Agent 2 opens its switch. The current rises to its previous, un-faulted level, and power is effectively restored to the system. 


\section{Chapter Eight - Conclusions}

\section{1 - Successes}

The complexity of a power system far surpasses the ability to be fully modeled in software. The Power System Simulator at WVU is a scaled replica of an antiquated distribution power system. The hardware aspects of a Smart Grid were identified and deployed to the PSS. By integrating industry standard protective devices, data acquisition, Multi-Agent System hosting, and reconfiguration capability, the PSS will serve as a test bed for a data rich and flexible distribution network.

SEL relays and computing platform installed on the simulator by Michael Spencer were configured to communicate and retrieve data from the system. All quantities necessary to efficiently operate a distribution network are available to the system operator and Agent-hosting hardware. The system is ready for coordination with Multi-Agent Systems.

The fundamental objective of a Multi-Grid Management System, fault identification, isolation, and reconfiguration, has been explored, and architecture capable of doing so effectively and efficiently has been established. The software is ready for further development, along with other MGMS functions such as Distributed Energy Management, Economic Dispatch, and others.

Coding for an Agent-host capable of interaction for reconfiguration, interfacing with an operator, and acquiring data from the system has been completed. Though not deployable to industry, the system provides a platform which can be used to test and further develop MGMS algorithms.

A case study has been employed which demonstrates the functionality of the MAS hosting and electronic switches. With further manipulation, this can become a full MGMS hosted in a real world environment.

All of the aforementioned aspects have been successfully integrated to the Power System Simulator. Protection and data acquisition through industry standard devices, MAS-hosting capability, and reconfiguration hardware has been applied and proven to suite the purposes of upgrading the Power System Simulator to a Smart Grid test bed. 


\section{2 - Future Work}

Additional electronic relays will be purchased for the full hybridization of the system. Easy installation guarantees the full deployment in a matter of weeks once the equipment is obtained. The SEL network must be fully wired, but all aspects have been considered, and it is now simply a matter of labor.

Although many of the fundamental obstacles have been hurdled, several aspects still remain. One hindrance is that the M52259Demokit cannot be used in industry. As the project intends to implement a fully exportable architecture, it fails in this aspect. However, in conjunction with the West Virginia Super Circuit project, a ruggedized computing platform has been selected and WVU will soon receive one such device for testing.

With further cooperation with industry representatives, the ruggedized computing platform will be used to host the MGMS as well as interface the electronic switches. Similarly, data acquired by the SEL relays will provide information about the system to be used in the algorithms. The interfacing of these devices and development of robust algorithms will be a challenge to overcome in the near future. 


\section{References}

Intelligent Agent. (2009, October). Retrieved September 20, 2011, from Wikipedia: http://en.wikipedia.org/wiki/Intelligent_agent

(2011, 10 26). Retrieved 10 15, 2011, from Schweitzer Engineering Laboratories: www.SELINC.com

CYME Power Engineering Software \& Solutions. (2011). Retrieved 10 7, 2011, from CYMFLOW Power Flow Analysis: http://www.cyme.com/software/cymecymflow/

Multi-Agent System. (2011, September 12). Retrieved September 20, 2011, from Wikipedia: http://en.wikipedia.org/wiki/Multi-agent_system

RTDS Hardware. (2011, 9 29). Retrieved 10 7, 2011, from RTDS Technologies: http://www.rtds.com/hardware/hardware.html

Barney L. Capehart, P. (2010, 6 10). Distributed Energy Resources. Retrieved 10 7, 2011, from Whole Building Design Guide: http://www.wbdg.org/resources/der.php

Belkacemi, R. (2011). Immune System Based Control and Intelligent Agent Design for Power System Applications. Morgantown.

Chouhan, S. (2008). Agent Based Modeling of Power Distribution Systems. Morgantown.

Grainger, J. J. (1994). Power Systems Analysis. New York: McGraw-Hill.

Inan, H. (2010). MGMS FLIR Use Case.

Inan, H. (2010). West Virginia Super Circuit Project Prelimiary Design Document, V2.

J. M. Solanki, N. N. (2007). A Multi-Agent Solution to Distribution Systems Restoration. IEEE Transactions on Power Systems, Vol 22, No. 3, (pp. 1026-1034).

J.M. Solanki, N. N. (2005). Reconfiguration for Resotration of Power Systems using Multi-Agent System. Proceedings of the 37th Annual North American Power Symposium, 390 - 395.

k. Huang, S. S. (2007). Decentralized Reconfiguration for Power Systems using Multi Agent Systems. 1st Annual IEEE Systems Conference, (pp. 1-6).

Nareshkumar, K. (2008). Application of Multi-Agents to Power Distribution Systems. Morgantown. 
R. Belkacemi, A. F. (2009). An Immune System Approach for Power System Automation and SelfHealing. Proc. of the IEEE Power Engineering Society. Seattle.

Schweitzer Engineering Laboratories. (2006). AcSELerator QuickSet Designer, SEL-5031 Instruction Manual. Washington, US.

Schweitzer Engineering Laboratories. (2006). SEL-751A Feeder Protection Relay Instruction Manual. Washington, US.

Spencer, M. J. (2010). Power Simulator Upgrade for Smart Grid Algorithm Development and Testing. Morgantown.

Srivatava, D. A. (2007). Agent Applications and their Future in Power Industry. IEEE Power Engineering Society General Meeting, (pp. 1-6).

T. Nagata, N. I. (2002). A new Power System Restoration Framework by Means of Multi-Agent Approach. IEEE/PES Transmission and Distribution Conference and Exhibition, (pp. 16951700).

T. Nagata, Y. T. (2004). Development of Bulk Power System Restoration Simulator by means of MultiAgent Approach. The 47th IEEE International Midwest Symposium on Circuits and Systems, Vol. 2, (pp. 337-340).

The Foundation for Physical Intelligent Agents. (n.d.). Retrieved from http://www.fipa.org

Weiss, G. (2000). Multiagent Systems: A Modern Approach to Distributed Artificial Intelligence. The MIT Press.

Wooldrigde, M. (2002). An Introduction to Multiagent Systems. John Wiley and Sons Ltd. 


\section{Appendix A: SEL Relay Demonstration Laboratory}

\section{Introduction}

This laboratory experiment is designed to demonstrate the functionality of SEL-734 Revenue Meter digital relay. The user is assumed to have basic knowledge of the Power System Simulator provided through EE336 - Electromechnical Energy Conversion and Systems Laboratory. At the end of the laboratory, a user will have learned to use the digital relays to view and acquire data from the system.

The user will first configure the SEL-3351 System Computing Platform to retrieve data from the SEL-734. Next, the PSS will be arranged to supply power to a residential load. Operating conditions will be verified between the analog meters on the PSS and the digital meters on the SEL-734. A fault will then be simulated, causing an Event Report to be triggered on the Revenue Meter. The user will then retrieve the Event Report and discuss their results.

\section{Procedure}

1. Access the SEL-3351 via the terminal on the left side of the Power System Simulator. Using the Windows XP Start Menu, load the SEL-5030 AcSELerator Quickset software.

2. In the Setup menu, select the 'Communication' icon. With the Active Connection Type set to Serial, select 'COM16: Xtreme PORT 4' in the Device tab. Settings should be as seen below, with RTS/CTS off, DRT on, and RTS on.

Click OK. Communication will be established with the SEL-734.

Com Settings for HMI through RS 232

\begin{tabular}{|l|c|}
\hline \multicolumn{1}{|c|}{ Parameter } & Value \\
\hline Data Speed (Baud) & 9600 \\
\hline Data Bits & 8 \\
\hline Stop Bits & 1 \\
\hline Parity & NONE \\
\hline
\end{tabular}


3. To access the phasors representation of the system, click on 'Tools' on the main window toolbar. Next, select 'HMI > HMI.' This should bring up the Human Machine interface of the SEL-734. From here, a user has access to the operating conditions of the Revenue Meter. When ready to proceed, select 'Phasors' on the left window pane. The system is now ready to observe the electrical quantities present on the 'Interconnection' panel of the Power System Simulator.

4. Now the simulator will be energized and prepared for the experiment. On the rightmost panel, set the VARIAC to 0 volts. Active the Simulator Main Power Supply (very bottom switch) as well as the Interconnect to Another System switch (3/4 up the panel). Also, provide the DC power for the protective system at the leftmost panel of the simulator (ensure that the power supply is active).

5. Next, power will be routed to the residential loads on the simulator. Starting at the right-most panel, Interconnection \& Sectionalizing, set the switches and breakers so that a conducting that is present through the bottom feeder and the left side of the Residential Distribution panel. However, ensure that both of the hand switches directly above the residential loads are turned off.

6. The loads at the bottom of this panel will be used to observe the operation of the relay; enable the third, fourth, and fifth loads from the left, but turn all others off. In the Industrial Loading panel, turn the VARIAC to $100 \%$. Enable the middle two switches, Power, and Heat, and the switch directly above; these loads will simulate a fault by creating a quick increase in current demand. A diagram of the enabled switches is shown below, with closed switches circled in green.

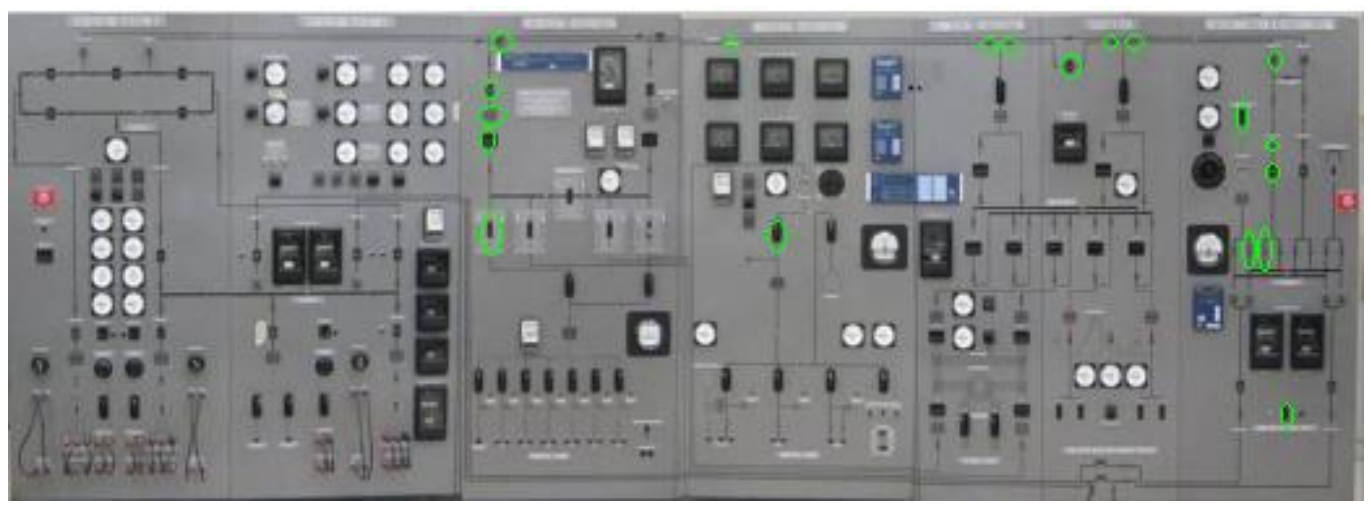

7. The system is ready to be observed in the event of a fault. Start by increasing the voltage on the Interconnect and Sectionalizing panel via the VARIAC rheostat. Next, enable the switch which 
feeds the Residential Loads from the bottom feeder (switch directly above the residential loads and to the left). The Phasors window on the HMI should display the voltage and current being delivered to the loads. Record this data.

8. Now simulate the fault by activating the switch directly above the Residential Loads and to the right. This should supply power to the Industrial Loads. The current will rise quickly, and the SEL-734 will identify the fault by the Overcurrent condition. Quickly view the Phasors on the HMI and record the data, then disable both load switches.

9. The SEL-734 is programmed to create an Event Report in the event of a fault. To access the file, first close the HMI screen on the SEL-3351. Next, select Tools $>$ Events $>$ Get Event Files. This will load a window displaying the event reports on the SEL-734. Select the most recent file with the checkbox, and then click the 'Get Selected Events' box on the right side of the screen. The file will be transferred, and you will be prompted to save the file.

10. Open the file from the directory from which you have saved it. The file displays the electrical quantities present on the simulator at the time of the simulated fault. Identify the occurrence of the fault and examine the voltage and current waveforms at this time.

11. Locate both the Industrial and Interconnect VARIACs once more and reduce the voltage to zero. Turn off the Simulator Main Power Supply, Interconnect to Another System, and 125VDC supply. The experiment is complete. 